\title{
Characterization and Correlation of Particle-Level Interactions to the Macroscopic Rheology of Powders, Granular Slurries, and Colloidal Suspensions
}

\author{
A.P. Poloski \\ R.C. Daniel \\ D.R. Rector \\ P.R. Bredt \\ E.C. Buck \\ Pacific Northwest National Laboratory \\ J.C. Berg \\ Department of Chemical Engineering \\ University of Washington \\ A.E. Sáez \\ Department of Chemical and Environmental Engineering \\ University of Arizona \\ Project Number - 90162 \\ Project Duration: FY04-FY06
}

September 29, 2006

Prepared for the U.S. Department of Energy

under Contract DE-AC05-76RL01830 


\title{
DISCLAIMER
}

This report was prepared as an account of work sponsored by an agency of the United States Government. Neither the United States Government nor any agency thereof, nor Battelle Memorial Institute, nor any of their employees, makes any warranty, express or implied, or assumes any legal liability or responsibility for the accuracy, completeness, or usefulness of any information, apparatus, product, or process disclosed, or represents that its use would not infringe privately owned rights. Reference herein to any specific commercial product, process, or service by trade name, trademark, manufacturer, or otherwise does not necessarily constitute or imply its endorsement, recommendation, or favoring by the United States Government or any agency thereof, or Battelle Memorial Institute. The views and opinions of authors expressed herein do not necessarily state or reflect those of the United States Government or any agency thereof.

\author{
PACIFIC NORTHWEST NATIONAL LABORATORY \\ operated by \\ BATTELLE \\ for the \\ UNITED STATES DEPARTMENT OF ENERGY \\ under Contract DE-AC05-76RLO1830
}




\title{
Characterization and Correlation of Particle-Level Interactions to the Macroscopic Rheology of Powders, Granular Slurries, and Colloidal Suspensions
}

\author{
A.P. Poloski \\ R.C. Daniel \\ D.R. Rector \\ P.R. Bredt \\ E.C. Buck \\ Pacific Northwest National Laboratory \\ J.C. Berg \\ Department of Chemical Engineering \\ University of Washington \\ A.E. Sáez \\ Department of Chemical and Environmental Engineering \\ University of Arizona \\ Project Number - 90162 \\ Project Duration: FY04-FY06
}

September 29, 2006

Prepared for the U.S. Department of Energy

under Contract DE-AC05-76RL01830

Pacific Northwest National Laboratory

Richland, Washington 99352 



\section{Executive Summary}

Hanford TRU tank sludges are complex mixtures of undissolved minerals and salt solids in an aqueous phase of high ionic strength. They show complex rheological behavior resulting from interactions at the macroscopic level, such as interparticle friction between grains in the coarse fraction, as well as from interactions at the nano-scale level, such as the agglomeration of colloidal particles. An understanding of phenomena such as interparticle friction and aggregate stability under shear will allow better control of Hanford TRU tank sludges being processed for disposal.

The project described in this report had two objectives. The first was to understand the physical properties and behavior of the Hanford transuranic (TRU) tank sludges under conditions that might exist during retrieval, treatment, packaging, and transportation for disposal at the Waste Isolation Pilot Plant (WIPP). The second objective was to develop a fundamental understanding of sludge physical properties by correlating the macroscopic behavior with interactions occurring at the particle/colloidal scale. These objectives were accomplished by: 1) measuring the physical and rheology properties of actual Hanford tank samples, 2) developing continuum models for coarse granular slurries, and 3) studying the behavior of colloidal agglomerates under shear and under ion irradiation.

The shear strength of coarse granular powders and slurries was studied to determine ways of characterizing (quantifying) the effect of interparticle friction and cohesion on incipient motion. Additional studies were performed to develop and validate a continuum model for cohesionless granular powders and slurries undergoing three-dimensional shear. With regard to incipient motion in dense slurries, the immersion depth and containing vessel diameter significantly impact shear strength . A model accounting for the effects of interparticle friction, slurry cohesion, vane size and immersion depth, and container geometry was derived using a saturating vertical stress profile. This model was able to adequately explain experimental observations with regards to variations in container size and vane immersion depth. The functional form of the Janssen formula provides a potential scaling relationship to apply small-scale shear vane results to engineering-scale applications dealing with coarse-grained slurry systems.

For granular systems undergoing three-dimensional flow, a constitutive model was developed to describe flows of cohesionless granular solids. The model is based on the postulate that the friction coefficient and the solids fraction in a moving granular material are exclusive functions of an inertial number, which represents the ratio of inertial to normal stress forces. The constitutive equation obtained has the same form as a multidimensional Bingham fluid model, albeit with an apparent viscosity and yield stress that depend on the vertical normal stress distribution. The model was applied to previously published experimental results dealing with three-dimensional shear flows of granular beds made up of cohesionless spherical particles. It adequately described the velocity profiles of the experimental granular flows using only a single adjustable parameter.

The continuum model was used to develop a vane rheometer technique for characterizing the flow behaviors of powders and slurries. Semi-quantitative characterization was achieved for quasi-static and intermediate granular flows. Separate approaches must be used at low and high vane rotational rates because of a change in the granular stress distribution resulting from fluidization. Granular solids were governed by a Janssen type normal stress distribution at low shear. At high rotational rates, the particles around the vane become fluidized, and the normal stress distribution influencing granular rheology was primarily lithostatic.

With regard to interactions occurring at the particle level, the behavior of colloids and colloidal 
agglomerates under irradiation and shear has been studied. It is believed that irradiation of colloidal materials present in Hanford TRU tank sludges by $\alpha$ particles may cause primary particle deformation and macroscopic aging effects on the slurry rheology. Ion irradiation of silica and bismuth oxide colloids, both of which are present in Hanford TRU tank sludges, produced mixed results. For silica irradiated with Xe, noticeable anisotropic deformation was observed for 100 $\mathrm{nm}$ spherical particles at fluences above $8.0 \times 10^{14}$ ions $/ \mathrm{cm}^{2}$. Smaller $50 \mathrm{~nm}$ silica particles were not anisotropically deformed by either Xe or He irradiation. Xenon irradiation of bismuth oxide effected significant expansion of the sample in the viewing plane and caused a crystalline to amorphous transition. The experimental results indicate that deformation of materials present in Hanford TRU sludges can occur as a result of ion bombardment. Examination of different particle sizes indicated that smaller particles could be resistant to anisotropic deformation.

A simulation methodology has been developed to predict the colloidal aggregate size distribution based on local flow conditions. The simulation uses the lattice Boltzmann/ lattice kinetics method to obtain the system hydrodynamics. This method is an improvement on conventional computational fluid dynamics in that it allows for parallel processing, reducing run time. The direct quadrature method of moments is used to solve the aggregate population balance equation at every computational node based on local flow and chemistry conditions. The resulting aggregate size distributions can then be used to predict the shear stress-strain rate relationships, which feed back into the flow solution.

Overall, the results of this study elucidate some of the phenomena influencing the flow properties of Hanford TRU tank sludges. The model described in this report provides a foundation by which the frictional, dilatational, and cohesive properties of granular powders and slurries may be characterized. Determination of these parameters and application of the continuum model facilitates scale-up of flow and stress dynamics from bench-scale to engineering-scale operations. Study of the stability and behavior of agglomerates under shear and studying of colloid aging effects as a result of ion-bombardment provide an understanding of how interactions occurring at the colloidal scale translate to macroscopic behavior. 


\section{Acknowledgments}

This work was supported by the U.S. Department of Energy (DOE) Environmental Management Science Program (EMSP) under Project No. 90162. Pacific Northwest National Laboratory is operated for DOE by Battelle under Contract DE-AC05-76RL01830. Collaborative efforts with Dr. John C. Berg at the University of Washington were supported under DOE grant DE-FG02-04ER63796. We wish to acknowledge the contributions of Amanda Bolta and Nathan Lester through the DOE Community College Institute (CCI). The CCI is a DOE-funded student research program for students from community colleges across the nation. DOE funding provided to the laboratories covers the costs of student stipends, housing allowances, and travel reimbursement. Part of the research presented was carried out in the Electron Microscopy Center at Argonne National Laboratory, which is supported by the DOE Office of Science under contract \#W-31-109-Eng-38. The authors also wish to thank Dr. Joel M. Tingey at Pacific Northwest National Laboratory for his helpful advice and technical support. 



\section{Abbreviations and Acronyms}

PBE Population Balance Equation

CCI Community College Institute

CH2M HILL CH2M HILL Hanford Group, Inc.

DOE U.S. Department of Energy

DQMOM The Direct Quadrature Method of Moments

EMSP Environmental Management Science Program

HLW High Level Waste

IVEM Intermediate Voltage Electron Microscope

MRI Magnetic Resonance Imaging

NIST National Institute of Standards and Technology

PNNL Pacific Northwest National Laboratory

RPM Revolutions Per Minute

TEM Transmission Electron Microscopy

TRU Transuranic (waste)

WIPP Waste Isolation Pilot Plant

WTP Hanford Waste Treatment and Immobilization Plant 


\section{Notation}

$a$

A

$A_{k}$

$A_{s}$

$A_{\tau}$

$b$

$B_{k}$

$\hat{B}$

$C_{c}$

$C_{D S}$

$C_{f}$

$d$

$f_{U D S}$

$F$

$\hat{F}$

$g$

$h$

$\hat{h}$

H

$H_{b}$

$\hat{H}$

I

$k_{B}$

K

$m_{\text {super }}^{f}$

$m_{\text {super }}^{o}$

M

$M_{b o t}$

$M_{\text {end }}$

$M_{r \theta}$

$M_{\text {shaft }}$

$M_{\text {side }}$

$M_{\text {top }}$

$M_{\text {total }}$

$M_{v}^{\max }$

$M_{z \theta}$

P

$P_{i j}$

da Cruz dilation law constant

surface area

fitting constant for consistency coefficient relationship

fitting constant for shear strength relationship

fitting constant for yield stress relationship

da Cruz friction law constant

fitting constant for consistency coefficient relationship

dimensionless cohesive force factor for shear strength analysis

number of contact points per unit area in a granular assembly

conversion factor for dissolved solids fraction

friction and geometrical interlocking coefficient of Shinohara

particle diameter

volume fraction of undissolved solids

single contact cohesive force between particles

dimensionless single contact cohesive force between particles

gravitational acceleration constant

vane immersion depth

dimensionless vane immersion depth (normalized to vane radius)

vane height

total depth of granular bed

dimensionless vane height (normalized to vane radius)

inertial number

Bingham consistency coefficient

Janssen coefficient (ratio of radial to vertical normal stress)

mass of supernatant after drying

mass of supernatant prior to drying

torque

torque acting on the bottom disk of vane rotation

averaged torque contribution from the vane ends (top/bottom)

torque acting on a radial surface

torque acting on the vane shaft

torque acting on the cylinder of vane rotation

torque acting on the top disk of vane rotation

total vane torque

maximum vane torque from shear strength testing

torque acting on a vertical surface

local pressure

component $i j$ of the diagonal normal stress tensor represent anisotropic pressure 


\begin{tabular}{|c|c|}
\hline$P_{r r}$ & radial normal stress \\
\hline$P_{z z}$ & vertical normal stress/pressure \\
\hline$P_{\infty}$ & Janssen saturation stress \\
\hline$r$ & radial coordinate \\
\hline$R$ & vane, disk, or cylinder radius (applies to rotating surfaces) \\
\hline$R_{0}$ & stationary ring radius for rotating disk experiments \\
\hline$R^{\text {cont }}$ & container radius \\
\hline$R_{s}$ & radius of vane shaft \\
\hline$R_{y}$ & radial extent of shear (radius of the yield surface) \\
\hline$S$ & local shear stress \\
\hline$v_{\theta}$ & azimuthal velocity component \\
\hline$V_{\text {super }}^{o}$ & volume of supernatant prior to drying \\
\hline$X$ & pressure distribution factor for shear strength analysis \\
\hline$X_{D S}$ & mass fraction of dissolved solids \\
\hline$X_{T S}$ & mass fraction of total solids \\
\hline$X_{U D S}$ & mass fraction of undissolved solids \\
\hline$\hat{X}$ & dimensionless pressure distribution factor for shear strength analysis \\
\hline$\hat{Y}$ & dimensionless torque for shear strength analysis \\
\hline$Y_{c}$ & yield parameter \\
\hline$z$ & vertical coordinate \\
\hline$\alpha$ & dimensionless bed depth \\
\hline$\beta$ & dimensionless group for rotating disk flow (ratio of granular yield to fluid stresses) \\
\hline$\beta^{\prime}$ & dimensionless group for rotating cylinder flow (ratio of granular yield to fluid stresses) \\
\hline$\delta_{i j}$ & component $i j$ of the Kronecker delta \\
\hline$\Delta \rho$ & buoyant bulk density \\
\hline$\Delta \hat{\rho}$ & dimensionless buoyant bulk density \\
\hline$\varepsilon$ & void fraction or interstitial fluid volume fraction \\
\hline$\phi$ & ratio of solids fraction to quasi-static solids fraction \\
\hline$\phi_{m}$ & reduced mass fraction of solids \\
\hline$\dot{\gamma}$ & second invariant of the rate of strain tensor \\
\hline$\dot{\gamma}_{0}$ & two-dimensional shear-rate for simple shear \\
\hline$\dot{\gamma}_{i j}$ & component $i j$ of the rate of strain tensor \\
\hline$\dot{\gamma}_{r \theta}$ & rate of strain at the $r-\theta$ plane \\
\hline$\dot{\gamma}_{z \theta}$ & rate of strain at the $z-\theta$ plane \\
\hline$\Gamma$ & dimensionless shear rate \\
\hline$\eta$ & dimensionless vertical coordinate \\
\hline$\kappa$ & dimensionless group for rotating cylinder flow (ratio of frictional to dilatational changes) \\
\hline$\lambda$ & Janssen screening length \\
\hline$\hat{\lambda}$ & dimensionless screening length (normalized to vane radius) \\
\hline$\mu$ & macroscopic coefficient of friction \\
\hline
\end{tabular}




\begin{tabular}{|c|c|}
\hline$\mu_{B}$ & apparent viscosity \\
\hline$\mu_{\min }^{*}$ & quasi-static macroscopic coefficient of friction for a granular material \\
\hline$\mu_{w}$ & coefficient of friction between a particle and a container wall \\
\hline$\mu_{w, k}$ & kinetic coefficient of friction between a particle and a container wall \\
\hline$\mu_{w, s}$ & static coefficient of friction between a particle and a container wall \\
\hline$\mu^{*}$ & macroscopic coefficient of friction for a granular material \\
\hline $\bar{\mu}^{*}$ & apparent coefficient of friction (neglects dynamic depth effects) \\
\hline$\mu^{\prime}$ & dimensionless apparent viscosity \\
\hline$v$ & solids fraction of a granular material \\
\hline$v_{\max }$ & quasi-static solids fraction of a granular material \\
\hline$\theta$ & angular coordinate \\
\hline$\rho$ & density \\
\hline$\rho_{D S}$ & density of the dissolved solids fraction \\
\hline$\rho_{p}$ & particle / material density \\
\hline$\rho_{\text {super }}$ & density of tank sludge supernatant \\
\hline$\rho_{\text {sus }}$ & density of the suspending (interstitial) fluid \\
\hline$\rho_{T S}$ & density of the total solids fraction \\
\hline$\rho_{U D S}$ & density of the undissolved solids fraction \\
\hline$\sigma_{i j}$ & component $i j$ of the total stress tensor \\
\hline$\tau$ & shear stress \\
\hline$\tau_{0}$ & yield stress \\
\hline$\tau_{i j}$ & component $i j$ of the deviatory stress tensor \\
\hline$\tau_{r \theta}$ & shear stress acting on the $r-\theta$ plane \\
\hline$\tau_{v}$ & shear stress on a rotating surface (vane, cylinder, or disk) \\
\hline$\tau_{v}^{\max }$ & shear strength \\
\hline$\tau_{y}$ & yield stress \\
\hline$\tau_{z \theta}$ & shear stress acting on the $z-\theta$ plane \\
\hline$T$ & dimensionless shear stress \\
\hline$T_{v}$ & dimensionless shear stress on a rotating surface (vane, cylinder, or disk) \\
\hline$\hat{\omega}$ & dimensionless local angular velocity normalized \\
\hline$\Omega$ & angular velocity of the vane or rotating cylinder \\
\hline$\zeta$ & dimensionless radial coordinate \\
\hline$\zeta_{y}$ & dimensionless radial extent of shear (dimensionless radius of the yield surface) \\
\hline
\end{tabular}




\section{Contents}

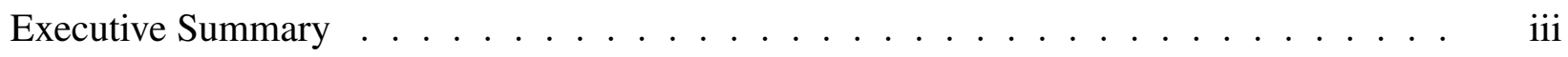

Acknowledgments ....................... $\quad$ v

Abbreviations and Acronyms $\ldots \ldots \ldots \ldots \ldots \ldots$ vii

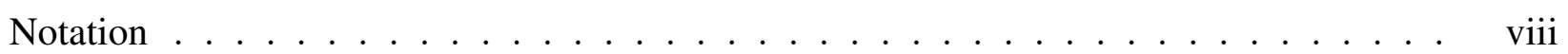

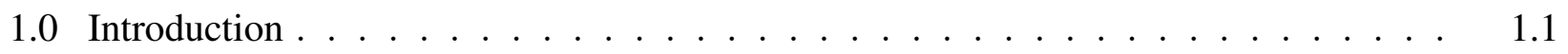

1.1 General Behavior - Coarse Powders and Slurries $\ldots \ldots \ldots$

1.2 General Behavior - Ion Irradiation Colloidal Particles $\ldots \ldots \ldots \ldots$

1.3 General Behavior - Colloidal Suspensions . . . . . . . . . . . . . . . . 1.2

1.4 Aim of this Report $\ldots \ldots \ldots \ldots \ldots \ldots \ldots \ldots$

2.0 Physical Properties of Hanford Tank Sludge $\ldots \ldots \ldots \ldots$

2.1 Tank Sample Source and Preparation $\ldots \ldots \ldots \ldots \ldots$

2.2 Supernatant Density and Dissolved Solids . . . . . . . . . . . . . . 2.3

2.3 Slurry Density, Total Solids Fraction, and Undissolved Solids Fraction and Density 2.4

2.4 Undissolved Solids Fraction and Density $\ldots \ldots \ldots$

2.5 Particle Size Distribution . . . . . . . . . . . . . . . . . . . . . . . 2.7

2.6 Particle Shape . . . . . . . . . . . . . . . . . . . . . . . . . 2.8

2.7 Macroscopic Rheology . . . . . . . . . . . . . . . . . . . . . . . . . 2.9

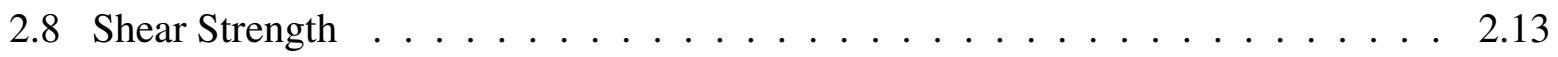

2.9 Conclusions . . . . . . . . . . . . . . . . . . . . . . . . . . . . . . . 2.14

3.0 Characterizing and Modeling Incipient Motion in Coarse Granular Slurries . . . . 3.1

3.1 Background . . . . . . . . . . . . . . . . . . . . . 3.1

3.2 Methods and Materials $\ldots \ldots \ldots \ldots \ldots \ldots \ldots \ldots$

3.3 Shear Strength Model $\ldots \ldots \ldots . . \ldots \ldots$

3.4 Results and Discussion . . . . . . . . . . . . . . . . . . . 3.10 
3.4.1 Dry Glass Bead Systems . . . . . . . . . . . . . . 3.11

3.4 .2 Glass Bead Slurry Systems . . . . . . . . . . . . . . 3.11

3.5 Conclusions . . . . . . . . . . . . . . . . . . . . 3.14

4.0 A Continuum Constitutive Model for Cohesionless Granular Flows . . . . . . . . . 4.1

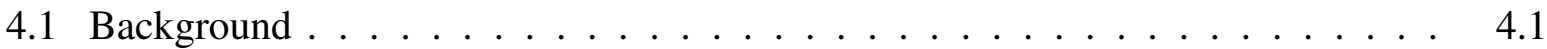

4.2 Constitutive Model . . . . . . . . . . . . . . . . . . . . 4.3

4.3 Flow over a Rotating Disk . . . . . . . . . . . . . . . . . 4.4

4.4 Flow between Concentric Cylinders . . . . . . . . . . . . . . . . . 4.10

4.5 Conclusion . . . . . . . . . . . . . . . . . . . 4.14

5.0 Characterizing Cohessionless Powders with Vane Rheology _ . . . . . . . . . . . . 5.1

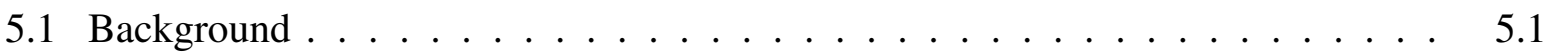

5.1.1 General Functionality of Vane Torque with Immersion Depth . . . . . . 5.4

5.1.2 Determination of Vane Shaft and End Effects . . . . . . . . . . . . 5.6

5.2 Methods and Materials . . . . . . . . . . . . . . . . . . 5.8

5.2.1 Measurement of Dry Glass Bead Flow Curves . . . . . . . . . . . . 5.8

5.2.2 Measurement of Static Granular Pressure Distribution . . . . . . . . . 5.9

5.3 Results and Discussion . . . . . . . . . . . . . . . . 5.10

5.3.1 Static Stress and Frictional Properties for the Glass Bead Powder . . . 5.10

5.3.2 Vane Tool Torque Profiles . . . . . . . . . . . . 5.12

5.3.3 Prediction of Vane Rheograms for the Dry Powder . . . . . . . . . . 5.19

5.3.4 Prediction of Vane Rheograms for Glass Bead Slurries . . . . . . . . . . 5.23

5.4 Conclusions . . . . . . . . . . . . . . . . . . 5.25

6.0 Anisotropic Deformation of Colloidal Particles by Ion Irradiation . . . . . . . . . . 6.1

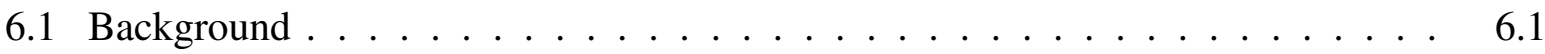

6.2 Methods and Materials . . . . . . . . . . . . . . . . 6.2 
6.3 Results and Discussion . . . . . . . . . . . . . . . 6.5

6.4 Conclusions . . . . . . . . . . . . . . . . . . . 6.7

7.0 Methodology for Colloidal Aggregation Modeling . . . . . . . . . . . . . . . 7.1

7.1 Description of the Direct Quadrature Method of Moments . . . . . . . . . 7.1

7.2 Description of the Lattice Kinetics Method . . . . . . . . . . . . . 7.3

7.3 Adaptation of the DQMOM Method to Shear-Activated Aggregation . . . . . . . 7.5

7.4 Validation of Modeling Methodology . . . . . . . . . . . . 7.6

8.0 Personnel Supported . . . . . . . . . . . . . . . . . . . . . 8.1

8.1 Project Support At PNNL $\ldots \ldots \ldots \ldots \ldots . \ldots \ldots$

8.2 University Collaborations . . . . . . . . . . . . . . 8.1

9.0 Publications . . . . . . . . . . . . . . . . . . . . . 9.1

10.0 Project Accomplishments . . . . . . . . . . . . . . . . . . . . . . . . . . 10.1

11.0 Future Work . . . . . . . . . . . . . . . . . . . . 11.1

12.0 References . . . . . . . . . . . . . . . . . . . . 12.1

Appendix A - Vane rheograms for Dense Slurries of Glass Beads in Kaolin Suspensions . A.1

Appendix B - University of Washington Collaborative Effort Update . . . . . . . . . . B.1 


\section{Figures}

2.1 Size distribution of undissolved solids in sonicated tank T-110 dilutions. . . . . . . 2.7

2.2 Size distribution of undissolved solids in sonicated tank T-204 dilutions. . . . . . . 2.7

2.3 Size distribution of undissolved solids in sonicated tank B-203 dilutions. . . . . . . 2.8

2.4 Size distribution of undissolved solids in sonicated tank T-203 dilutions. . . . . . . 2.8

2.5 Yield stress for tank samples as a function of the mass fraction of undissolved solids. 2.10

2.6 Bingham consistency coefficient for tank samples as a function of the mass fraction of undissolved solids. . . . . . . . . . . . . . . . . 2.10

2.7 Yield stress (data) as a function of the reduced mass fraction solids. The solid line corresponds to a best-fit of Eq. (2.10). . . . . . . . . . . . . . . . . 2.12

2.8 Bingham consistency (data) as a function of the reduced mass fraction solids. The solid line corresponds to a best-fit of Eq. (2.11) . . . . . . . . . . . . . . 2.12

2.9 Shear strength for tank samples as a function of the mass fraction of undissolved solids. 2.13

2.10 Shear strength (data) as a function of the reduced mass fraction solids. The curve corresponds to a best-fit of Eq. (2.12). The dashed portion is an extrapolation of Eq. (2.12) to concentrations lower than studied herein and represents a region of uncertainty with regard to the applicability of the fitting relationship. . . . . . . . . . . . . . 2.14

3.1 A schematic illustrating several significant dimensions and concepts for calculating shear strength using the shear vane method. The inset shows the typical output from a shear vane measurement; time is on the abscissa while shear stress calculated from Equation 3.1 is on the ordinate. . . . . . . . . . . . . . . . .

3.2 Torque measurements as a function of vane depth for the systems described in Table 3.2. 3.10

3.3 Torque measurements as a function of vane depth for Systems A-F described in Table 3.2. The data have been non-dimensionalized according to Equation 3.12 with $K=0.39$ and $\mu_{w}=0.95$ for Systems A-E. . . . . . . . . . . . . . . 3.11

3.4 Torque measurements as a function of vane depth for Systems F-I described in Table 3.2. The data have been non-dimensionalized according to Equation 3.12 with $K=0.43$

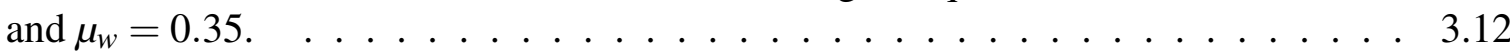

3.5 Torque measurements as a function of vane depth for System I described in Table 3.2. This system consists of a mixture of glass beads dispersed in $35 w t \%$ kaolin clay slurry. The system was allowed to sediment for $1200 \mathrm{~min}$ before measuring shear strength. These results indicate a settled glass bead layer at an immersion depth of approximately $5 \mathrm{~cm} .3 .14$

4.1 Geometry of the rotating disk system. . . . . . . . . . . . . . . 4.5 
4.2 Experimental (symbols) [Cheng et al. (2006)] and fitted (solid lines) angular velocity profiles for rotating disk flow in a bed with $\alpha=0.88$. The symbols and accompanying lines correspond to: $\eta=0.792(\bullet), \eta=0.6952$ (०), $\eta=0.6072(\boldsymbol{\square}), \eta=0.5104$ $(\square), \eta=0.2376(\Delta)$. The solid lines represent the best fit to the whole set of data, obtained for $\beta=50$. The dashed lines are results of a discrete simulation (details in Cheng et al. (2006), and references therein). . . . . . . . . . . . . . . 4.8

4.3 Comparison of various models to experimental data for rotating disk flow in a bed with $\alpha=0.88$. For symbols see Figure 4.2. The solid lines correspond to predictions for $\eta=0.792$; the dashed lines are for $\eta=0.510$. Predictions for $\beta \rightarrow \infty$ at $\eta=0.510$ coincide with the $\zeta$ axis. . . . . . . . . . . . . . . .

4.4 Experimental (symbols) [Cheng et al. (2006)] and predicted (solid lines, $\beta=50$ ) angular velocity profiles for rotating disk flow in a bed with $\alpha=0.4$. The symbols and accompanying lines correspond to: $\eta=0.316(\bullet), \eta=0.228(\circ), \eta=0.140(\boldsymbol{\square}) . \quad$. 4.10

4.5 Angular velocity at the surface of the bed and cell axis for the experiments reported by Cheng et al. (2006): experimental data (•), model predictions (solid line) using $\beta=$ 15 (no adjustable parameters, see text). The dashed line represents the predictions for a Newtonian fluid. . . . . . . . . . . . . . . . . . . . . . 4.11

4.6 Experimental (symbols) [Mueth et al. (2000)] and fitted (solid lines) angular velocity (a) and solids fraction (b) profiles for flow between concentric cylinders of mustard seeds $\left(d=1.8 \mathrm{~mm}, v_{\max }=0.58\right)$ at $\eta=0.284$. The fits correspond to $\beta^{\prime}=40$ and $\kappa=$ 3.2. The oscillation in the experimental results is caused by the fact that measurements span just a few particles in the radial direction. . . . . . . . . . . . . . . . 4.14

4.7 Experimental (symbols) [Mueth et al. (2000)] and fitted (solid lines) angular velocity (a) and solids fraction (b) profiles for flow between concentric cylinders of poppy seeds $\left(d=0.8 \mathrm{~mm}, v_{\max }=0.67\right)$ at $\eta=0.290$. The fits correspond to $\beta^{\prime}=85$ and $\kappa=2.5 .4 .15$

5.1 In a typical vane rheology test, a vane tool of radius $R$ and height $H$ is immersed to a depth $h$ in a sediment layer confined by a cylindrical wall with a radius of $R_{\text {cont }}$. The vane is rotated at a constant angular velocity $\Omega$, and the total torque $M_{\text {total }}$ required to maintain motion is recorded. Rotation of the vane sweeps out a cylinder, which is considered to be the shear surface. The total torque acting on the vane tool results from shear forces on the cylinder top, bottom, side, and shaft. . . . . . . . . . . . .

5.2 An illustration of the experimental setup used for the vane rheology tests. A 2 L Pyrex $^{\circledR}$ beaker filled with a $203 \mu \mathrm{m}$ powder is placed on an adjustable lab-jack directly beneath the measuring system. The beaker is raised until the vane is immersed to the desired test depth. Steady-state stepped-flow curves were operated over $0.03 \mathrm{rad} / \mathrm{s}$ to $50 \mathrm{rad} / \mathrm{s}$ to determine the glass bead torque-rotational rate profiles. . . . . . . . . . 
5.3 Measured (a) radial normal stress and (b) static shear strength (torque) as a function of immersion depth for a 203- $\mu$ m glass-bead powder. Curves in part (a) correspond to pressure profiles calculated using Janssen's law [see Eq. (5.1)] with $K$ and $\mu_{w}$ regressed from direct stress measurement (solid line: $K=0.6, \mu_{w}=0.7$ ) and those inferred from analysis of torque profiles for Systems A-C in Chapter 1 (dashed line: $K=0.39, \mu_{w}$ $=0.95)$. Curves in part (b) are torque profiles calculated from direct stress measurements (solid line: $C_{f}=1.8, K=0.6, \mu_{w}=0.7$ ) and from the results presented in Chapter 1 (dashed line: $\left.C_{f}=2.2, K=0.39, \mu_{w}=0.95\right) \ldots \ldots \ldots . \ldots \ldots$

5.4 Quasi-static torque measurements presented in accordance with Eq. (5.19). Data calculated from measurements of equilibrium torque on a vane rotating at $0.03 \mathrm{rad} / \mathrm{s}$ in a $203-\mu \mathrm{m}$ glass-bead powder. Immersion depths from $10 \mathrm{~mm}$ to $90 \mathrm{~mm}$ were tested using tools of varying geometry $(R=8 \mathrm{~mm}, H=2-32 \mathrm{~mm})$. Linear regression on the entire data set yields $\mu_{\text {min }}^{*}$ of 0.8 . The lower and upper extremes of the set are bound by $\mu_{\text {min }}^{*} 0.5$ and 1.0 , respectively. . . . . . . . . . . . . 5.13

5.5 Total torque acting on an $8-\mathrm{mm} \times 16-\mathrm{mm}$ vane immersed in a $203-\mu \mathrm{m}$ glass-bead powder as a function of vane rotational rate at various immersion depths. The transition in flows that occurs from $0.03 \mathrm{rad} / \mathrm{s}$ to $50 \mathrm{rad} / \mathrm{s}$ is not smooth, but appears to occur in a number of steps. Below $0.5 \mathrm{rad} / \mathrm{s}$, the torque is independent of rotational rate. At $2 \mathrm{rad} / \mathrm{s}$, a strong increase in torque is observed. This increase becomes more moderate at $8 \mathrm{rad} / \mathrm{s}$.

5.6 Total torque acting on a $8-\mathrm{mm} \times 16-\mathrm{mm}$ vane immersed in a $203-\mu \mathrm{m}$ glass-bead powder as a function of depth at various points in the flow regime. Profiles show lithostatic behavior up to $50 \mathrm{~mm}$. At deeper immersions, deviation from linearity results from interaction of the vane stresses with the container walls. . . . . . . . . . . .

5.7 Torque contributions for a 3-mm shaft immersed in a $203-\mu m$ glass-bead powder as a function of rotational rate at various depths (a) and as a function of depth at specific points in the torque evolution (b). Similar to total torque, profiles show lithostatic behavior up to about $50 \mathrm{~mm}$ and deviate from Eq. (5.9) [quadratic dependence on $h$, represented by dashed lines] at deeper depths because of the interaction of stress chains with the container. . . . . . . . . . . . . . . . . . . 5.16

5.8 Averaged disk torque contributions for an 8 - $\mathrm{mm} \times 16-\mathrm{mm}$ vane immersed in a 203$\mu \mathrm{m}$ glass-bead powder as a function of rotational rate at various depths (a) and as a function of depth at various flow regimes (b). Dashed lines illustrate the linear / zero intercept dependence of torque on $h$ [Eq. (5.15)]. . . . . . . . . . . . . . 5.17

5.9 Total vane torque as a function of vane height at an immersion depth of $20 \mathrm{~mm}$ (a) and $90 \mathrm{~mm}$ (b). Dashed curves are the best-fit lines used to determine vane end effects. Extrapolations at low vane immersion depths follow the expected quadratic functionality [Eq. (5.10)] while extrapolations at deeper immersion depth are complicated by significant point-to-point scatter.

5.10 Lithostatic slope for vane end effects from $10 \mathrm{~mm}$ to $50 \mathrm{~mm}$ as a function of rotational rate. Beyond $1 \mathrm{rad} / \mathrm{s}$, the lithostatic slope appears to be independent of rotational rate and behaves as a Coulomb solid with a bulk friction coefficient equal to the limit of kinetic friction at zero flow (i.e., $\mu_{\min }^{*}=0.8$; dashed line) . . . . . . . . 5.18 
5.11 Cylindrical (side) torque contributions for an 8- $\mathrm{mm} \times 16-\mathrm{mm}$ vane immersed in a 203$\mu \mathrm{m}$ glass bead powder as a function of rotational rate at various depths (a) and as a function of depth at various flow regimes (b). Dashed lines illustrate the linear dependence of torque on $h$ [Eq. (5.10)] . . . . . . . . . . . . . . . . . . . . 5.19

5.12 Comparison total vane torque for the 203- $\mu$ m glass bead powder at various immersion depths with predictions (solid lines) based on a Janssen stress profile [Eq. (5.1)] for $\Omega<0.5 \mathrm{rad} / \mathrm{s}$ and on a lithostatic stress profile [Eq. (5.4)] for $\Omega>8 \mathrm{rad} / \mathrm{s}(K=0.6$, $\left.\mu_{w}=0.7\right)$

5.13 Comparison of total torque predictions at high rotational rates against experimental data for $60 \mathrm{~mm}$. Torque calculations based on a lithostatic pressure distribution (solid line, $b=5$ ) provides a better representation of the data than calculations based on a Janssen pressure distribution (dashed line, $b=15$ ). All fits use $K=0.6, \mu_{w}=0.7$, and $\mu_{\text {min }}^{*}=$

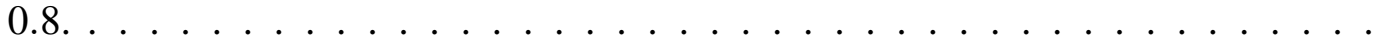

5.14 Quasi-static torque measurements for the glass bead slurry presented in accordance with Eq. (5.19). Data are calculated from measurements of equilibrium torque on a vane rotating at $0.03 \mathrm{rad} / \mathrm{s}$ in a $203-\mu \mathrm{m}$ glass-bead slurry in water. Immersion depths from $10 \mathrm{~mm}$ to $90 \mathrm{~mm}$ were tested using an $8-\mathrm{mm} \times 16-\mathrm{mm}$ vane tool. Analysis (solid curve) of the data yields $\mu_{\text {min }}^{*}=0.55, K=0.6$, and $\mu_{w}=0.55$.

5.15 Comparison total vane torque for the $203-\mu \mathrm{m}$ glass bead slurry at various immersion depths with predictions (solid lines) based on a Janssen stress profile [Eq. (5.1)] for $\Omega<0.5 \mathrm{rad} / \mathrm{s}$ and on a lithostatic stress profile [Eq. (5.4)] for $\Omega>8 \mathrm{rad} / \mathrm{s}(K=0.6$, $\left.\mu_{w}=0.7\right)$.

6.1 Basic illustration of the anisotropic shape change experienced by a spherical colloid undergoing ion irradiation. Here, the ion beam strikes the particle from the top, causing it to expand laterally and contract on the axis parallel to the ion beam.

6.2 TEM of 100-nm colloidal silica $\left(\mathrm{SiO}_{2}\right)$. There is little obvious fusing between adjacent particles. Primary particles are nearly spherical and are relatively monodisperse. 6.3

6.3 TEM of 40-nm polydisperse colloidal bismuth oxide $\left(\mathrm{Bi}_{2} \mathrm{O}_{3}\right)$. but aggregated. Analysis of the micrograph suggests that the bismuth oxide is crystalline. . . . . . . .

6.4 Sample stage setup for ion irradiation experiments. Stage is tilted $-15^{\circ}$ with respect to the electron beam. Ion beam is strikes the sample at a $15^{\circ}$ angle . . . . . . . .

6.5 50-nm spherical silica $\left(\mathrm{SiO}_{2}\right)$ (a) before irradiation and (b) after irradiation (fluence: $1.2 \times 10^{15}$ ions $/ \mathrm{cm}^{2}$ ) with $1 \mathrm{MeV} \mathrm{Xe} \mathrm{Xe}^{+}$ions . . . . . . . . . . . . .

6.6 Evidence of sputtering during irradiation of 50-nm spherical silica $\left(\mathrm{SiO}_{2}\right)$ with $1 \mathrm{MeV}$ $\mathrm{Xe}^{+}$ions

6.7 100-nm spherical silica $\left(\mathrm{SiO}_{2}\right)$ (a) before irradiation and (b) after irradiation (fluence: $8.0 \times 10^{14}$ ions $/ \mathrm{cm}^{2}$ ) with $1 \mathrm{MeV} \mathrm{Xe}^{+}$ions . . . . . . . . . . . . . .

6.8 40-nm spherical bismuth oxide $\left(\mathrm{Bi}_{2} \mathrm{O}_{3}\right)$ (a) before irradiation and (b) after irradiation (fluence: $2.0 \times 10^{14}$ ions $/ \mathrm{cm}^{2}$ ) with $1 \mathrm{MeV} \mathrm{Xe}^{+}$ions . . . . . . . . . . . 
$6.950-\mathrm{nm}$ spherical silica $\left(\mathrm{SiO}_{2}\right)$ (a) before irradiation and (b) after irradiation (fluence: $1.0 \times 10^{15}$ ions $/ \mathrm{cm}^{2}$ ) with $500 \mathrm{keV} \mathrm{He}^{+}$ions $\ldots \ldots \ldots \ldots$

A.1 Vane rheographs for a 54-wt $\%$ glass bead - 46-wt $\%$ kaolin matrix slurry over vane immersion depths of $1 \mathrm{~cm}$ to $9 \mathrm{~cm} \ldots \ldots \ldots \ldots \ldots$. . . . . . . . . . . .

A.2 Vane rheographs for a $64-\mathrm{wt} \%$ glass bead - 36-wt $\%$ kaolin matrix slurry over vane immersion depths of $1 \mathrm{~cm}$ to $9 \mathrm{~cm} \ldots \ldots \ldots \ldots$. . . . . . . . . . . . .

A.3 Vane rheographs for a $74-w t \%$ glass bead $-26-w t \%$ kaolin matrix slurry over vane immersion depths of $1 \mathrm{~cm}$ to $9 \mathrm{~cm} \ldots \ldots$ 


\section{Tables}

2.1 TRU sample composite identification numbers. . . . . . . . . . . . . . 2.2

2.2 TRU sample dilutions prepared. . . . . . . . . . . . . . . . . . 2.2

2.3 Density (in $\mathrm{g} / \mathrm{mL})$ of the supernatant for select tank dilutions. . . . . . . . . . . . . 2.4

2.4 Mass fraction of dissolved solids for select tank dilutions. . . . . . . . . . . . . . . 2.4

2.5 Density (in $\mathrm{g} / \mathrm{mL}$ ) of the dried dissolved solids for select tank dilutions. . . . . . . . 2.4

2.6 Mass fraction of total dried solids for select tank dilutions. . . . . . . . . . . . . . 2.5

2.7 Slurry density (in $\mathrm{g} / \mathrm{mL}$ ) of tank sludges at select dilutions. . . . . . . . . . . . 2.5

2.8 Density (in $\mathrm{g} / \mathrm{mL}$ ) of dried total solids for select tank dilutions. . . . . . . . . . . 2.5

2.9 Mass fraction of undissolved solids for tank samples and dilutions. . . . . . . . . . 2.6

2.10 Calculated density (in $\mathrm{g} / \mathrm{mL}$ ) of dried undissolved solids for select tank dilutions. 2.6

2.11 Volume fraction of undissolved solids for tank samples and dilutions. . . . . . . . . 2.6

2.12 Aspect ratio for undissolved solids for select tank dilutions. . . . . . . . . . . . . . 2.9

2.13 Mass fraction of solids at maximum packing for select tank dilutions. . . . . . . . . 2.11

2.14 Volume fraction of solids at maximum packing for select tank dilutions. . . . . . . 2.11

3.1 Measured Torque at Various Rotational Rates with $1.6 \times 1.6$ (diameter $\times$ height, $\mathrm{cm} \times$ $\mathrm{cm}$ ) Shear Vane at Constant Depth . . . . . . . . . . . . . . 3.6

3.2 Systems investigated as a function of vane depth. . . . . . . . . . . . . . . . . 3.7

3.3 Linear regression results for the systems described in Table 3.2 . . . . . . . . . . 3.13

5.1 Low rotational rate torque measured using an $8 \times 16(R \times H, m m \times m m)$ shear vane at an immersion depth of $20 \mathrm{~mm}$. These measurements verify that data in Fig. 5.4 correspond to the quasi-static flow region. . . . . . . . . . . 5.12 



\subsection{Introduction}

This project had two primary objectives. The first was to understand the physical properties and behavior of select Hanford tank sludges under conditions that might exist during retrieval, treatment, packaging, and transportation for disposal at the Waste Isolation Pilot Plant (WIPP). The second objective was to develop a fundamental understanding of these sludge suspensions by correlating the macroscopic properties with particle interactions occurring at the colloidal scale. The specific tank wastes considered herein are contained in thirteen Hanford tanks including three double-shell tanks (DSTs) (AW-103, AW-105, and SY-102) and ten single-shell tanks (SSTs) (B-201 through B-204, T-201 through T-204, T-110, and T-111). At the outset of the project, these tanks were designated as potentially containing transuranic (TRU) process wastes that would be treated and disposed of in a manner different from the majority of the tank wastes.

These objectives were accomplished by: 1) developing continuum models for coarse granular slurries that allow prediction of the rheology of the Hanford TRU tank sludges under conditions that might exist during treatment and packaging, 2) exploring the particle and aggregate dynamics at the microscopic level to facilitate better understanding of how shear influences the slurry / suspension structure during transport, and 3) developing relationships between colloidal parameters and bulk physical properties such as yield stress and shear modulus. The majority of work concerning TRU sludge physical properties, granular slurry rheology, and aggregate structure dynamics was performed by Pacific Northwest National Laboratory (PNNL) staff. Granular rheology efforts were assisted through a collaborative effort with Professor A. Eduardo Sáez from the University of Arizona. Work relating to the development of relationships between colloidal parameters and bulk physical properties was accomplished through a collaborative effort with Professor John C. Berg from the University of Washington [DOE grant DE-FG02-04ER63796] and will be reported in a later publication. The results of this research effort enhance the existing understanding of flow properties of coarse slurries and colloidal suspensions. In addition, the knowledge gained and capabilities developed during this effort will aid in the development and optimization of techniques to process the wastes at various DOE sites.

Hanford TRU tank sludges are complex mixtures of undissolved mineral and salt solids. The liquid wastes initially introduced to the tanks were acidic high level waste (HLW) from spent nuclear fuel reprocessing and did not contain a large quantity of solids. However, subsequent neutralization of the HLW to prevent tank corrosion and additional waste treatment processes resulted in the precipitation of significant solid material. The aqueous suspending phase remaining has high ionic strength due to the presence of dissolved salt solids. The size of the primary particles and particle aggregates is highly polydisperse, containing both colloidal and coarsegrained particles. In general, while the macroscopic behavior of such slurries depends on the suspension concentration, the physical properties of the materials comprising the dispersed and suspending phases often derive from interactions that occur on the particle level. For suspensions of fine particulate matter, surface forces are dominant. Understanding the macroscopic behavior here requires understanding of colloidal forces such as van der Waals attraction between primary particles, particle aggregation kinetics, and aggregate breakdown and reformation under shear. On the other hand, the dynamics of coarse suspensions and slurries depend more on particle mechanical interactions such as friction, dissipation of energy during particle collisions, cohesion of particles, and viscous dissipation through motion of the suspending medium around the particles and particle assemblies. Particle-particle mechanical interactions are particularly important for highly dense slurries. 


\subsection{General Behavior - Coarse Powders and Slurries}

Course granular powders and slurries exhibit highly non-Newtonian behavior because of frictional and collisional interactions between individual grains. Even for non-cohesive materials, the range of granular behavior is diverse. For powders and slurries at rest, incipient motion can only be achieved when sufficient force has been applied to overcome static frictional contacts within the material (Fayed and Otten 1997, Nedderman 1992). Frictional failure involves dilating the granular network of particles so that the individual grains may slip past their neighbors. After mobilization, three flow regimes are typically observed for cohesionless granular solids and slurries: quasi-static, intermediate, and kinetic. Slow or poorly fluidized granular flows are dominated by interparticle friction and are termed "quasi-static", owing to the fact that the local shear stress is proportional to pressure and virtually independent of shear rate. Rapid or highly fluidized granular flows are dominated by particle collisions and have been traditionally described by molecular kinetic theories (Campbell 1990, Goldhirsch 2003, Jenkins and Richman 1985). Intermediate flows fall between the quasi-static and kinetic regions; here fluidization and/or shear are strong enough to dissipate significant energy through particle collisions, but do not completely eliminate the continuous particle contact network typical of quasi-static flows. The intermediate region has been the recent subject of attention by Tardos et al. (2003), MiDi (2004), da Cruz et al. (2005), and Jop et al. (2006). Both Jop et al. and da Cruz et al. postulate constitutive laws for intermediate flows of granular materials. The proposal of da Cruz et al. has been adapted into a continuum representation by Sáez et al. (2006), whose work shall be detailed in Chapter 4.

\subsection{General Behavior - Ion Irradiation Colloidal Particles}

Recently published data indicate that colloid particles can deform under the influence of ion irradiation. In the immediate vicinity of the ion path, the colloid melts and solidifies on a time scale of nanoseconds. This melting and solidification process creates a cumulative stress on the colloid particle that deforms the particle over time. For TRU tank sludges, a high degree of $\alpha$ radiation may provide a similar mechanism for deformation. Using the published model with

${ }^{238} \mathrm{Pu} \alpha$ radiation rather than Xe ion irradiation, a fluence of approximately $1 \times 10^{15} \mathrm{ions} / \mathrm{cm}^{2}$ would be required before significant deformation is observed. Considering the relatively high degree of $\alpha$-emitting isotopes in the sludge and the creation date of these wastes (1940s-1950s), fluences of this magnitude are expected in the actual waste and provide a potential mechanism for the creation of oblate spheroids in the TRU sludge.

\subsection{General Behavior - Colloidal Suspensions}

A significant portion of Hanford tank waste exhibits pseudoplastic (shear thinning) rheological behavior that has important implications for the design of retrieval, transport and processing processes. The shear thinning behavior, in most cases, is due to the aggregation of colloidal particles. The agglomerate size distribution is controlled by the relative rates of aggregation and breakup of these agglomerates as a function of local shear and solution chemistry. Recent advances have been made in the area of predicting the aggregate size distribution (Marchisio and Fox 2005) and relating this to the rheological behavior of the suspension.

\subsection{Aim of this Report}

Mechanical and colloidal particle interactions have been extensively studied at the microscopic level. The challenge in applying the results of these studies to complex materials like TRU tank sludges is 1) having an appropriate means of characterizing the physical and mechanical proper- 
ties and 2) having expressions through which one can incorporate these measured properties into predictions of physical properties and mass / momentum transport. Extrapolation of bench-scale measurements to engineering-scale applications also presents significant difficulty for dense granular systems, whose properties (such as flowability) are determined by interactions of the grains with the container walls.

The work presented in this report forms a basis for understanding and characterizing the influence of colloidal and frictional interactions on bulk slurry properties. With respect to mass transport, continuum relationships that account for the effect of container geometry are developed. While applied to bench-scale operations, they are sufficiently general to allow application to the engineering-scale processes involved in TRU tank sludge retrieval, treatment, packaging, and transportation. While the target application of this work is waste retrieval and disposal, the studies herein examine model systems such as dry glass bead powders, kaolinite suspensions, or mixtures of coarse powders in a clay suspension. As such, the findings can be applied to orthogonal processes concerned with mass transport and physical properties of dilute suspensions or concentrated slurries.

The report is structured as follows: Chapter 2 discusses the various physical and rheological properties of Hanford tank sludges motivating the studies presented in later chapters. Chapters 3 to 5 discuss coarse granular rheology. Specifically, Chapter 3 discusses the characterization and modeling of incipient motion in powders and dense slurries. Chapter 4 presents a continuum model for the characterization and modeling of dynamic granular flows. The formulation is independent of geometry. Studies of granular flows around rotating cylinders and over rotating disks are used to validate the approach. Chapter 5 focuses mainly on the characterization of granular rheology using a shear vane tool, which is a simple rheometric technique typically used to measure slurry shear strength. Based on the continuum model developed in Chapter 4, the results presented in Chapter 5 are directly applicable to mixing and agitation of dense slurries.

Later chapters discuss the deformation and agglomeration of colloidal particles under the influence of shear. Chapter 6 discusses a brief study of anisotropic deformation of silica and bismuth oxide colloids. Chapter 7 describes research regarding the shear thinning behavior of colloidal suspensions, with a focus on the formation and growth of colloidal aggregates. Here, the Direct Quadrature Method of Moments (DQMOM) is introduced, and a model for shear-activated aggregation is provided. 



\subsection{Physical Properties of Hanford Tank Sludge}

This chapter reports on efforts to characterize Hanford tank wastes with regard to their macroscopic rheology and to the properties of the dispersed phase that influence rheology. The first task of the EMSP project reported herein was to gain basic physical property data on actual tank sludges. To maximize the usefulness and applicability of this data, the tank sludges selected for study are significant to the current Hanford cleanup process.

At the outset of this project, CH2M HILL Hanford Group, Inc. (CH2M HILL) was in the process of identifying and developing supplemental process technologies to accelerate the Hanford tank waste cleanup mission. A range of technologies were being evaluated to allow disposal of Hanford waste types, including those tentatively designated as TRU tank wastes. For TRU tank sludges, the planned tank retrieval process involved mobilization of tank sludge sediments with a water jet. The water-diluted waste would then be pumped to down-stream processes including drying and packing for transport to WIPP.

At the time, thirteen Hanford waste tanks were identified as containing constituents that could have allowed that tank waste to be designated as TRU waste. These tanks included three doubleshell tanks (DSTs) (AW-103, AW-105, and SY-102) and ten single-shell tanks (SSTs) (B-201 through B-204, T-201 through T-204, T-110, and T-111). Although these tank wastes have been subsequently designated as high-level waste, this report will refer to these wastes as TRU wastes to be consistent with previous project updates. The current retrieval process for high level wastes still involves mobilization with liquid jets. Mobilized waste is pumped down-stream to evaporation, filtration, and vitrification units. As such, an understanding of the rheological behavior of these sludges under dilution is still important.

Samples from four of the TRU tanks have been previously studied at PNNL in support of the CH2M HILL retrieval activities. These are tanks B-203, T-203, T-204, and T-110. PNNL developed correlations between rheological properties and dilution level for tank retrieval purposes. These relations have been reported to $\mathrm{CH} 2 \mathrm{M}$ HILL in previously published reports (Tingey et al. 2003, Tingey 2004).

While these correlations are suitable for engineering work, they are not suitable for research purposes. The goal of the research presented in this chapter was to augment these correlations with additional experimental data and to prepare updated correlations. To this end, supplemental experimental data including the density of dried dissolved solids and particle size and shape have been obtained.

In the following sections, select measurements of physical properties relevant to the rheological behavior of TRU tank sludge are presented. These include the size distribution of solid particulate matter comprising the tank waste, the density and shape of those particles, and the macroscopic rheological behavior of the TRU sludge. Each of the particle properties listed (density, size, and shape) can influence macroscopic rheology. Particle size determines if interactions between particles are primarily mechanical (e.g., frictional contact between large particles) or colloidal (e.g., van der Waals attraction between small particles). Particle size and density determine, in part, the stability with respect to settling of the undissolved solids in the sludge. As will be seen in later sections, density also determines the lithostatic pressure in continuously contacted sedimented sludge layers. As described in Sherwood (1997), the jamming limit (volume fraction) of a granular bed is determined by the particle shape. Both sedimentation and rheology are influenced by particle shape anisotropy; indeed, shear thinning behavior in suspensions of anisotropic particles can occur due to favorable alignment of the particle with the flow field. 
With regard to these properties and to the macroscopic rheology, the effect of tank waste dilution is examined to better understand process rheology during sludge mobilization.

\subsection{Tank Sample Source and Preparation}

Four waste samples from tanks B-203, T-203, T-204, and T-110 were supplied by the 222-S Laboratory. Details regarding the quantity of material received, pre-treatment, and dose rates of these samples may be found in Tingey et al. (2003). Table 2.1 lists sample identification and tracking information. In general, samples will be referenced to their particular tank number or jar number.

Diluted tank wastes were prepared by adding water to the sludge and homogenizing the sample. In general, four dilution levels were employed: DL1, DL2, DL3, and DL4. These correspond to the "initial", "30 wt\%" solids, "1:1", and "4:1" samples dilutions listed in Table 3.3 of Tingey et al. (2003). The "initial" dilution was only prepared for tank T-110 waste, which had dried during transport to and storage at PNNL. The dilution was prepared to make a sludge that had a concentration more representative of the actual concentration when the sample was initially taken and that would allow it to pass the paint filter test. The "30 wt \%" dilutions were prepared for all four tank samples. The " $1: 1$ " and " $4: 1$ " designations describe the ratio of water to sludge sample. Both dilution levels were prepared for all tank samples. DL0 refers to tank material used "as-received". Table 2.2 outlines the TRU sample dilutions prepared and their identification markers from Tingey et al. (2003).

The following sections present measurements of the physical and rheological properties of the sludge samples. Because of sample volume limitations, only select dilutions were tested. All physical properties measurements were conducted at room temperature. The ultimate goal of the sludge density and supernatant/solids fractions calculations outlined in the following sections was to determine the concentration of undissolved solids in the diluted waste. Undissolved solids are important to the rheology of dense slurries, as interactions between solid particulates (moderated by the interstitial liquid) determine the overall flow behavior. This will become apparent when the relationships for sludge macroscopic rheology are introduced toward the end of this chapter,

Table 2.1. TRU sample composite identification numbers.

\begin{tabular}{ccc}
\hline Lab Core Identifier & Tank Number & Jar Number \\
\hline S03T000053 & B-203 & 19031 \\
S03T000081 & T-203 & 19032 \\
S03T000200 & T-110 & 19025 \\
S03T000148 & T-204 & 19026 \\
\hline
\end{tabular}

Table 2.2. TRU sample dilutions prepared.

\begin{tabular}{ccc}
\hline Dilution Level & PNNL-14365 Dilution Category & Tank Samples Employed \\
\hline DL0 & "As-received" & T-110, T-204, B-203, T-203 \\
DL1 & Initial & T-110 \\
DL2 & $30 \mathrm{wt} \%$ & T-110, T-204, B-203, T-203 \\
DL3 & $1: 1$ & T-110, T-204, B-203, T-203 \\
DL4 & $4: 1$ & T-110, T-204, B-203, T-203 \\
\hline
\end{tabular}


as all are strong functions of the fraction of undissolved solids.

\subsection{Supernatant Density and Dissolved Solids}

The supernatant density and mass fraction of dissolved solids in the supernatant for the diluted tank waste samples were determined by aliquoting $1 \mathrm{~mL}$ of waste supernatant for each tank/dilution into $20 \mathrm{~mL}$ separate centrifuge cones. The quantity of diluted supernatant added to the cone was determined by comparing the cone mass before and after sample addition. The density of the supernatant, $\rho_{\text {super }, i}$, for tank/dilution sample $i$ was then calculated by

$$
\rho_{\text {super }, i}=\frac{m_{\text {super }}^{o}}{V_{\text {super }}^{o}}
$$

where $m_{\text {super }}^{o}$ and $V_{\text {super }}^{o}$ are the mass and volume of supernatant added to the centrifuge cone, respectively. Next, the mass of dissolved solids contained in the waste supernatant was determined by heating the samples with a heating lamp until the free liquid had evaporated. Samples were subsequently placed in a drying oven at $105^{\circ} \mathrm{C}$ until the remaining liquid was removed. Sample dryness was verified by confirming that the sample mass did not change appreciably (i.e., by $0.02 \mathrm{~g}$ ) upon continued heating over a 24 hour period. The dry sample mass was determined by re-weighing the centrifuge cone. Then the mass fraction of dissolved solids was calculated as the ratio of dry sample mass, $m_{\text {super }}^{f}$ to the initial mass:

$$
X_{D S, i}=\frac{m_{\text {super }}^{f}}{m_{\text {super }}^{o}}
$$

Here $X_{D S, i}$ represents the mass fraction of dissolved solids for the tank/dilution $i$. The density of the dried dissolved solids recovered from the drying operation was subsequently determined using an AccuPyc 1330 pycnometer from the Micromeritics Instrument Corporation (Norcross, GA 30093-1877).

Tables 2.3, 2.4, and 2.5 show the measured supernatant density, dried dissolved solids density, and dissolved solids mass fraction for the various dilution levels. Due to test volume limitations and time constraints, only select dilution levels were measured directly. In general, only the DL3 samples were examined. With regard to the fraction of dissolved solids, the DL3 samples for tanks T-110, T-203, and B-203 and the DL4 sample for tank T-204 were examined. Dissolved solids fractions for dilution levels not tested were calculated using

$$
X_{D S, i}^{*}=\frac{C_{D S, i}}{1-X_{D S, r e f}}
$$

where $X_{D S, r e f}$ is the reference dissolved solids fraction and $C_{D S, i}$ is the conversion factor for dissolved solids fraction for the particular tank sample/dilution level from Tingey et al. (2003). It should be noted that the $*$ superscript is used to differentiate dissolved solid fractions calculated from those directly measured. 
Table 2.3. Density (in $\mathrm{g} / \mathrm{mL}$ ) of the supernatant for select tank dilutions.

\begin{tabular}{ccccccc}
\hline Tank Number & Jar Number & DL0 & DL1 & DL2 & DL3 & DL4 \\
\hline T-110 & 19025 & DL0 & DL1 & DL2 & 1.10 & DL4 \\
T-204 & 19026 & & & & 1.07 & 1.05 \\
B-203 & 19031 & & & & 1.12 & \\
T-203 & 19032 & & & & 1.11 & \\
\hline
\end{tabular}

Table 2.4. Mass fraction of dissolved solids for select tank dilutions.

\begin{tabular}{ccccccc}
\hline Tank Number & Jar Number & DL0 & DL1 & DL2 & DL3 & DL4 \\
\hline T-110 & 19025 & $23.2 \%$ & $17.7 \%$ & $11.6 \%$ & $6.8 \%$ & $2.7 \%$ \\
T-204 & 19026 & $17.0 \%$ & & $16.1 \%$ & $8.5 \%$ & $3.4 \%$ \\
B-203 & 19031 & $13.2 \%$ & & $9.0 \%$ & $6.6 \%$ & $2.6 \%$ \\
T-203 & 19032 & $13.2 \%$ & & $11.1 \%$ & $6.6 \%$ & $2.7 \%$ \\
\hline
\end{tabular}

Table 2.5. Density (in $\mathrm{g} / \mathrm{mL}$ ) of the dried dissolved solids for select tank dilutions.

\begin{tabular}{|c|c|c|c|c|c|c|}
\hline Tank Number & Jar Number & DL0 & DL1 & DL2 & DL3 & DL4 \\
\hline $\mathrm{T}-110$ & 19025 & & & & 2.19 & \\
\hline T-204 & 19026 & & & & 2.12 & \\
\hline B-203 & 19031 & & & & 2.22 & \\
\hline $\mathrm{T}-203$ & 19032 & & & & 2.37 & \\
\hline
\end{tabular}

At DL3, the density of the supernatant is around $1.1 \mathrm{~g} / \mathrm{mL}$ for all tanks, which is about $5 \%$ lower than observed in Tingey et al. (2003). The dried dissolved solids represent the material (typically salt solids) dissolved in the supernatant; they have densities between $2.1 \mathrm{~g} / \mathrm{mL}$ and $2.4 \mathrm{~g} / \mathrm{mL}$. It should be noted that these measurements were taken using DL3 samples. Even though the samples are dried, the dilution level impacts the measured density because of dissolution of solids and carry-over to the interstitial liquid prior to drying.

\subsection{Slurry Density, Total Solids Fraction, and Undissolved Solids Fraction and Density}

The mass fraction of total solids for the four tank waste samples have been reported in Tingey et al. (2003) and are reproduced in Table 2.6. This information was expanded upon by determining the density of both the tank slurry and total (dried) solids contained therein. Here, an aliquot of homogenized tank material was placed into a graduated centrifuge cone. The total slurry density was determined by dividing the total mass of material in the cone by the volume occupied by the sludge. The results are reported in Table 2.7. Next, the sample was heated to dryness using the procedure for dried dissolved solids outlined in the previous section. The density of the solids remaining after drying was determined using the AccuPyc 1330 pycnometer. The results of this analysis, shown in Table 2.8, exhibit total dried solids densities between $2.4 \mathrm{~g} / \mathrm{mL}$ and $2.8 \mathrm{~g} / \mathrm{mL}$. 
Table 2.6. Mass fraction of total dried solids for select tank dilutions.

\begin{tabular}{ccccccc}
\hline Tank Number & Jar Number & DL0 & DL1 & DL2 & DL3 & DL4 \\
\hline T-110 & 19025 & $62.2 \%$ & $48.3 \%$ & $29.2 \%$ & $15.3 \%$ & $6.4 \%$ \\
T-204 & 19026 & $31.4 \%$ & & $29.4 \%$ & $14.6 \%$ & $6.1 \%$ \\
B-203 & 19031 & $40.4 \%$ & & $28.5 \%$ & $19.7 \%$ & $8.4 \%$ \\
T-203 & 19032 & $34.3 \%$ & & $30.6 \%$ & $16.3 \%$ & $6.3 \%$ \\
\hline
\end{tabular}

Table 2.7. Slurry density (in $\mathrm{g} / \mathrm{mL}$ ) of tank sludges at select dilutions.

\begin{tabular}{ccccccc}
\hline Tank Number & Jar Number & DL0 & DL1 & DL2 & DL3 & DL4 \\
\hline T-110 & 19025 & 1.31 & 1.50 & 1.25 & 1.13 & 1.04 \\
T-204 & 19026 & 1.17 & & 1.28 & 1.13 & 1.05 \\
B-203 & 19031 & 1.28 & & 1.25 & 1.17 & 1.05 \\
T-203 & 19032 & 1.21 & & 1.28 & 1.14 & 1.04 \\
\hline
\end{tabular}

Table 2.8. Density (in $\mathrm{g} / \mathrm{mL}$ ) of dried total solids for select tank dilutions.

\begin{tabular}{|c|c|c|c|c|c|}
\hline Tank Number & Jar Number & DL0 DL1 & DL2 & DL3 & DL4 \\
\hline $\mathrm{T}-110$ & 19025 & & & 2.46 & \\
\hline T-204 & 19026 & & & 2.76 & \\
\hline B-203 & 19031 & & & 2.63 & \\
\hline T-203 & 19032 & & & 2.78 & \\
\hline
\end{tabular}

\subsection{Undissolved Solids Fraction and Density}

Using the fraction of total solids (Table 2.6), the mass fraction of undissolved solids in each waste sample/dilution were determined though,

$$
X_{U D S, i}=1-\frac{1-X_{T S, i}}{1-X_{D S, i}}
$$

where $X_{U D S, i}$ and $X_{T S, i}$ represent the mass fraction of undissolved and total solids in sample $i$, respectively. The results of this calculation are reported in Table 2.9. It is then possible, based on the measured densities of the total solids and dissolved solids, to calculate the density of the undissolved solids using the following equation:

$$
\rho_{U D S, i}=\frac{\rho_{T S, i}-\left[\frac{\left(1-X_{U S D, i}\right) X_{D S, i}}{X_{U S D, i}+\left(1-X_{U S D, i}\right) X_{D S, i}}\right] \rho_{D S, i}}{1-\left[\frac{\left(1-X_{U S D, i}\right) X_{D S, i}}{X_{U S D, i}+\left(1-X_{U S D, i}\right) X_{D S, i}}\right]}
$$


Here, $\rho_{U D S, i}$ and $\rho_{T S, i}$ are the calculated dried undissolved solids density and measured dried total solids density for tank sample/dilution $i$, respectively. The results of this calculation are shown in Table 2.10. Relative to the dissolved solids, the undissolved solids show a higher density variation. The range of densities observed here bound $2.6 \mathrm{~g} / \mathrm{mL}$ to $3.6 \mathrm{~g} / \mathrm{mL}$.

Next, the volume fraction of undissolved solids may be determined by

$$
f_{U D S, i}=X_{U D S, i}\left(\frac{\rho_{\text {slurry }, i}}{\rho_{U D S, i}}\right)
$$

where $\rho_{\text {slurry,i }}$ is the measured density of the slurry (Table 2.7). Calculated volume fractions of undissolved solids are presented in Table 2.11. Because the density information for dried undissolved solids are limited to DL3, volume fractions at other dilution levels were calculated using the DL3 density alone. As such, they should be considered as estimates only, as the density of undissolved solids at DL3 may not be representative of the density of undissolved solids at different dilution levels. It would have been preferable to determine $\rho_{U D S}$ for all dilution levels; however, time and sample volume constraints limited the number of samples examined.

Table 2.9. Mass fraction of undissolved solids for tank samples and dilutions.

\begin{tabular}{ccccccc}
\hline Tank Number & Jar Number & DL0 & DL1 & DL2 & DL3 & DL4 \\
\hline T-110 & 19025 & $50.7 \%$ & $37.2 \%$ & $19.9 \%$ & $9.1 \%$ & $3.8 \%$ \\
T-204 & 19026 & $17.4 \%$ & & $15.9 \%$ & $6.6 \%$ & $2.8 \%$ \\
B-203 & 19031 & $31.3 \%$ & & $21.4 \%$ & $14.0 \%$ & $6.0 \%$ \\
T-203 & 19032 & $24.3 \%$ & & $22.0 \%$ & $10.4 \%$ & $3.8 \%$
\end{tabular}

Table 2.10. Calculated density (in $\mathrm{g} / \mathrm{mL}$ ) of dried undissolved solids for select tank dilutions.

\begin{tabular}{|c|c|c|c|c|c|c|}
\hline Tank Number & Jar Number & DLO & DL1 & DL2 & DL3 & DL4 \\
\hline $\mathrm{T}-110$ & 19025 & & & & 2.65 & \\
\hline $\mathrm{T}-204$ & 19026 & & & & 3.53 & \\
\hline B-203 & 19031 & & & & 2.80 & \\
\hline $\mathrm{T}-203$ & 19032 & & & & 3.02 & \\
\hline
\end{tabular}

Table 2.11. Volume fraction of undissolved solids for tank samples and dilutions.

\begin{tabular}{ccccccc}
\hline Tank Number & Jar Number & DL0 & DL1 & DL2 & DL3 & DL4 \\
\hline T-110 & 19025 & $25.2 \%$ & $21.0 \%$ & $9.4 \%$ & $3.9 \%$ & $1.5 \%$ \\
T-204 & 19026 & $5.8 \%$ & & $5.8 \%$ & $2.1 \%$ & $0.8 \%$ \\
B-203 & 19031 & $14.3 \%$ & & $9.6 \%$ & $5.9 \%$ & $2.2 \%$ \\
T-203 & 19032 & $9.8 \%$ & & $9.4 \%$ & $3.9 \%$ & $1.3 \%$ \\
\hline
\end{tabular}




\subsection{Particle Size Distribution}

The size distribution of solid particulate matter comprising the tank waste was measured using a Malvern Mastersizer 2000 (Southborough, MA 01772, USA) with a Hydro $\mu$ P sample dispersion unit operated at 3000 RPM to maintain sample suspension. Experiments were conducted at room temperature. During the particle size measurements, the diluted sludge samples were continuously sonicated to break apart weak flocs. For these experiments, only DL2 and DL4 for tanks T-110, T-204, B-203, and T-203 were tested. The results are shown in Figs. 2.1 to 2.4.

The particle size distribution of the TRU tank sludges is predominantly a bimodal distribution of particles with one peak at approximately $10 \mu \mathrm{m}$ and another peak at approximately $100 \mu \mathrm{m}$. The one exception appears to be tank B-203, which shows a relatively unimodal distribution of particles around the $10 \mu \mathrm{m}$ range. In general, as the level of water dilution increases the relative amount of $100 \mu \mathrm{m}$ mode particle decreases suggesting solids dissolution in this size range or a change in particle agglomeration due to the change in suspending phase ionic strength.

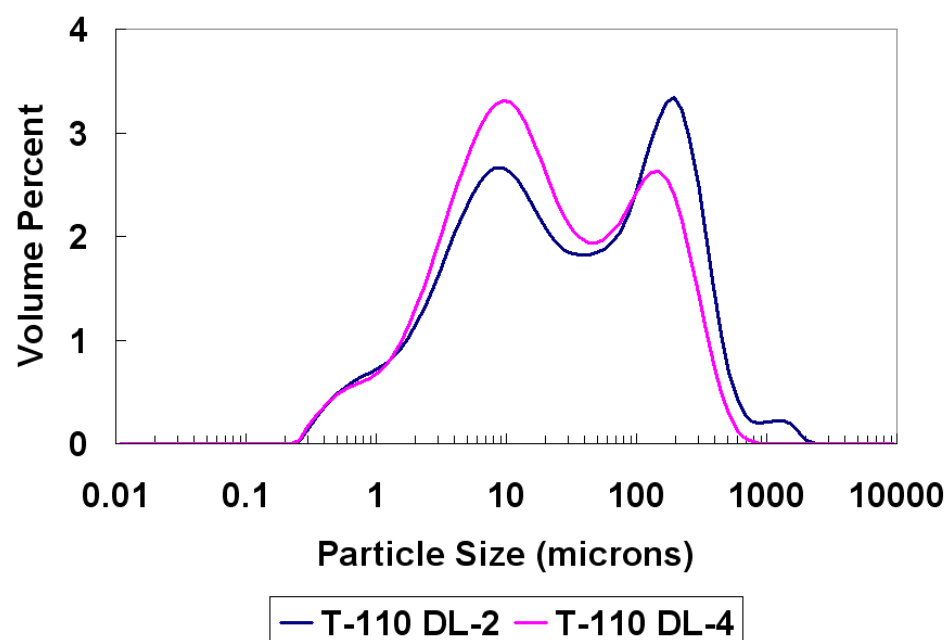

Figure 2.1. Size distribution of undissolved solids in sonicated tank T-110 dilutions.

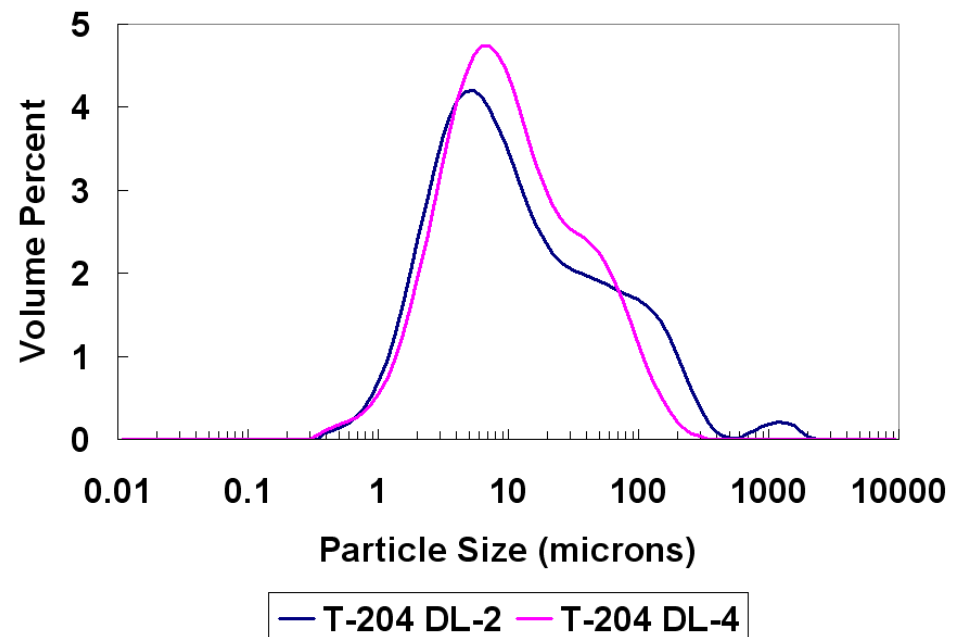

Figure 2.2. Size distribution of undissolved solids in sonicated tank T-204 dilutions. 


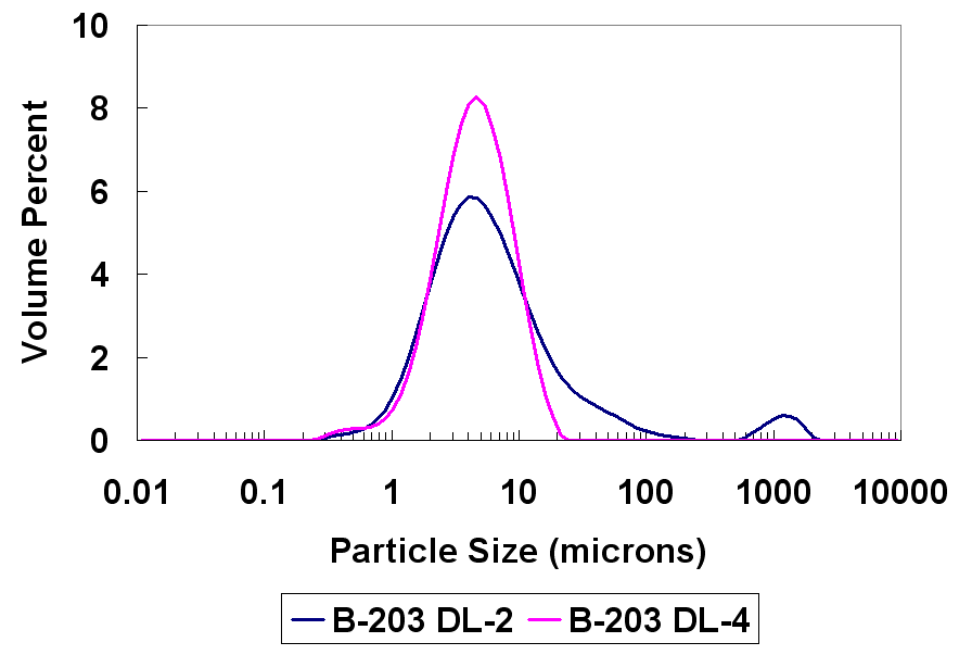

Figure 2.3. Size distribution of undissolved solids in sonicated tank B-203 dilutions.

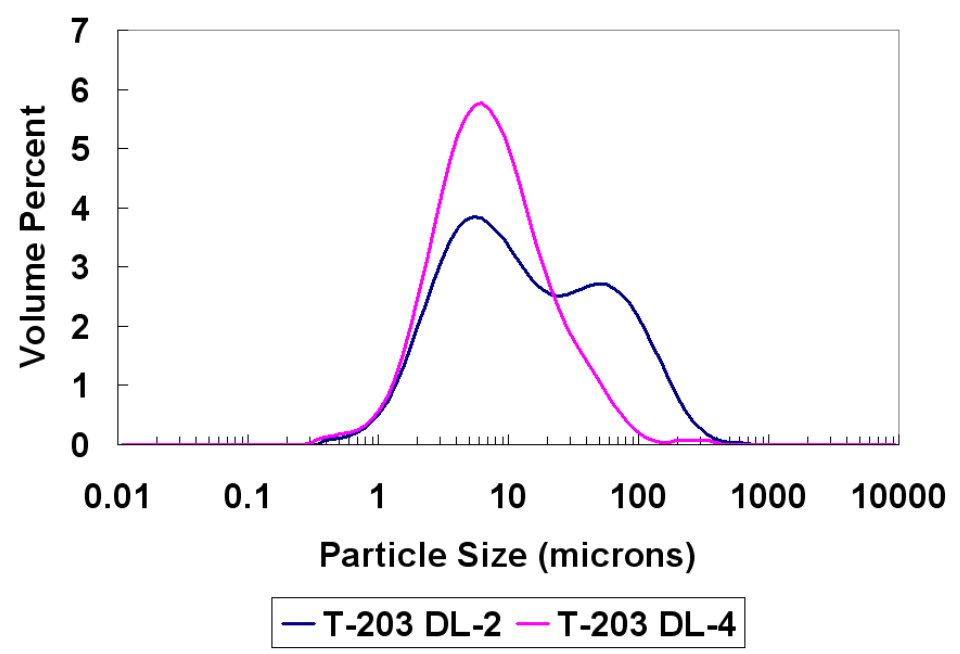

Figure 2.4. Size distribution of undissolved solids in sonicated tank T-203 dilutions.

\subsection{Particle Shape}

The aspect ratio of undissolved solids comprising the tank waste was determined using the results of particle size analysis from the Malvern Mastersizer 2000. Because they are based on the particle size measurements outlined above, aspect ratios were determined for only dilutions DL2 and DL4. Here the particles are treated as ellipsoids, and the aspect ratio represents the ratio of the length of the axis of symmetry to the diameter of the meridian circle. Prolate particles have aspect ratios greater than unity, while oblate particles have aspect ratios less than unity. The aspect ratio is calculated as the ratio of the volume fraction of solids in the sample analyzed to the volume fraction measured by the Mastersizer:

$$
A R=\frac{f_{U D S, \text { actual }}}{f_{U D S, \text { measured }}}
$$

Here, $A R$ is the aspect ratio and $f_{U D S, \text { actual }}$ and $f_{U D S \text {,measured }}$ are the actual and measured volume fraction of undissolved solids analyzed, respectively. 
Table 2.12. Aspect ratio for undissolved solids for select tank dilutions.

\begin{tabular}{|c|c|c|c|c|c|c|}
\hline Tank Number & Jar Number & DL0 & DL1 & DL2 & DL3 & DL4 \\
\hline $\mathrm{T}-110$ & 19025 & & & 6 & & 21 \\
\hline $\mathrm{T}-204$ & 19026 & & & 9 & & 29 \\
\hline B-203 & 19031 & & & 12 & & 2 \\
\hline $\mathrm{T}-203$ & 19032 & & & 6 & & 14 \\
\hline
\end{tabular}

Table 2.12 presents the results of the aspect ratio analysis. The particle shape data indicate that the sludges consist of particles with an aspect ratio significantly greater than unity. This suggests the presence of prolate spheroid particles. Spheroidal particles with either large (as in this case) or small aspect ratios form sedimented beds with relatively low volume fractions at maximum packing $(<30 \%)$. Measurements of the volume fraction of solids at maximum packing (see Table 2.14) show volume fractions less than $30 \%$, and appear to be consistent with the observation of anisotropic particle shape made using the Malvern Mastersizer. Since maximum packing of solids is a significant parameter in the rheological models, processes influencing the particle shape in tank wastes are of interest for further investigated. This is the driving force behind studies of anisotropic deformation presented in Chapter 6.

At DL2, the aspect ratios range from 6 to 12. Upon further dilution, the aspect ratio for most of the tanks tends to increase by at least a factor of two. Based on the decrease in the large particle peak seen in the particle size distributions, the increase in aspect ratio upon dilution would tend to suggest the smaller particles have higher aspect ratios than the larger particles. The one exception is the waste from B-203, which shows a reduction in aspect ratio upon dilution. It is speculated that this anomalous behavior relates to the difference in B-203's relatively unimodal particle size distribution relative to the other tanks.

\subsection{Macroscopic Rheology}

Sludge rheology was characterized as a function of the mass fraction of undissolved solids for various dilutions. Tests were carried out using a rotational rheometer equipped with a concentric cylinder sensor system and verified against National Institute of Standards and Technology (NIST) traceable standards. The analysis is similar to that described in Chapter 6 of Tingey et al. (2003). It is found that actual TRU sludge flow curve data follows the Bingham Plastic rheological model. Here, the shear stress $\tau$ is related to the shear rate $\dot{\gamma}$ by

$$
\tau=\tau_{0}+k_{B} \dot{\gamma}
$$

In this equation, $\tau_{0}$ is the yield stress and $k_{B}$ is the Bingham consistency coefficient. Both the consistency coefficient and yield stress were determined from sludge rheograms as a function of undissolved solids concentration. The results are shown in Figs. 2.5 and 2.6.

These yield stress and consistency data were fit with correlations previously developed in the rheological literature (Landel et al. 1965, Dabak and Yucel 1987, Slatter 1997). The Bingham plastic consistency and yield stress may be expressed as functions of the reduced mass fraction 


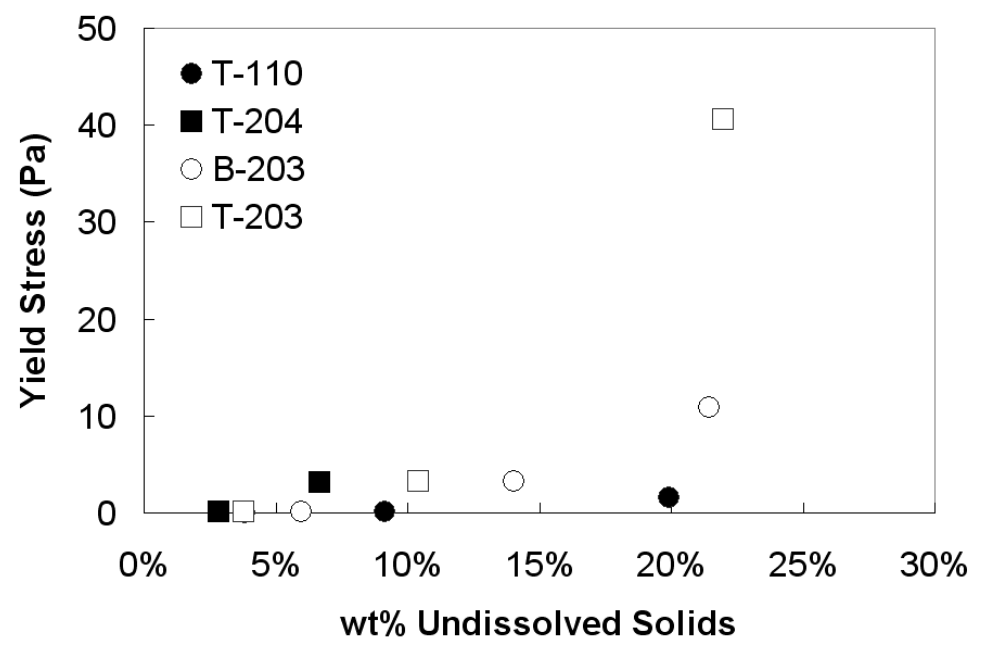

Figure 2.5. Yield stress for tank samples as a function of the mass fraction of undissolved solids.

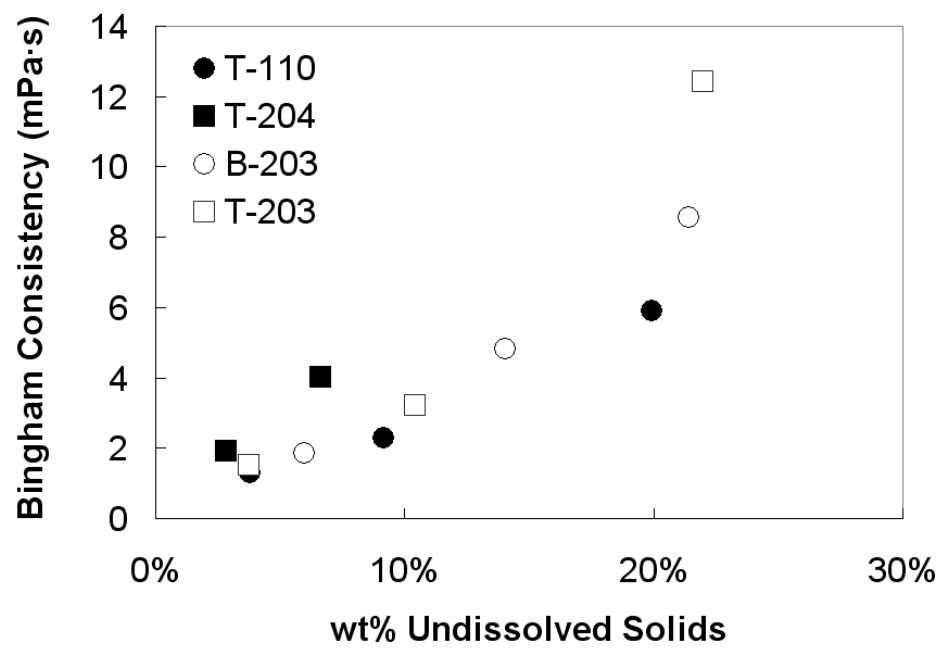

Figure 2.6. Bingham consistency coefficient for tank samples as a function of the mass fraction of undissolved solids. 
solids, $\phi_{m}$, which is defined as

$$
\phi_{m}=\frac{\text { mass fraction solids }}{\text { mass fraction solids at maximum packing }}
$$

Equation 2.9 requires knowledge of the mass fraction of solids at maximum packing. This is determined by centrifuging the slurry to form the most dense sediment layer possible. Table 2.13 lists the mass fraction solids at maximum packing for the four tank sludges studied herein. This can also be translated into a volume fraction solids at maximum packing, which is presented in Table 2.14 for the four tank sludges. It should be noted that all the volume fractions are less than $30 \mathrm{vol} \%$, which is indicative of anisotropic particle shape.

The yield stress was fit to the formula,

$$
\tau_{0}=A_{\tau} \frac{\phi_{m}^{3}}{1-\phi_{m}}
$$

while the Bingham consistency coefficient was fit to,

$$
k_{B}=A_{k}\left(1-\phi_{m}\right)^{-B_{k}}
$$

Here, $A_{\tau}, A_{k}$, and $B_{k}$ are fitting constants. The results of the fitting analysis are shown in Figs. 2.7 and 2.8. The reduced mass fraction correlations are able to adequately describe the variation of yield stress and Bingham consistency with solids loading using at most two adjustable parameters. A best-fit of the data yields $A_{\tau}=18 \pm 4 \mathrm{~Pa}$ and $A_{k}=1.5 \pm 0.2 \mathrm{mPa} . \mathrm{s}$, $B_{k}=1.7 \pm 0.1$.

Table 2.13. Mass fraction of solids at maximum packing for select tank dilutions.

\begin{tabular}{ccccccc}
\hline Tank Number & Jar Number & DL0 & DL1 & DL2 & DL3 & DL4 \\
\hline T-110 & 19025 & $50.7 \%$ & $44.2 \%$ & $41.5 \%$ & $39.1 \%$ & $33.5 \%$ \\
T-204 & 19026 & $18.9 \%$ & & $18.7 \%$ & $14.4 \%$ & $13.3 \%$ \\
B-203 & 19031 & $34.7 \%$ & & $34.9 \%$ & $31.0 \%$ & $30.0 \%$ \\
T-203 & 19032 & $26.1 \%$ & & $30.5 \%$ & $23.5 \%$ & $20.3 \%$ \\
\hline
\end{tabular}

Table 2.14. Volume fraction of solids at maximum packing for select tank dilutions.

\begin{tabular}{ccccccc}
\hline Tank Number & Jar Number & DL0 & DL1 & DL2 & DL3 & DL4 \\
\hline T-110 & 19025 & $25.2 \%$ & $25.0 \%$ & $19.7 \%$ & $16.6 \%$ & $13.2 \%$ \\
T-204 & 19026 & $6.3 \%$ & & $6.8 \%$ & $4.6 \%$ & $3.9 \%$ \\
B-203 & 19031 & $15.8 \%$ & & $15.6 \%$ & $12.9 \%$ & $11.3 \%$ \\
T-203 & 19032 & $10.5 \%$ & & $13.0 \%$ & $8.9 \%$ & $7.0 \%$ \\
\hline
\end{tabular}




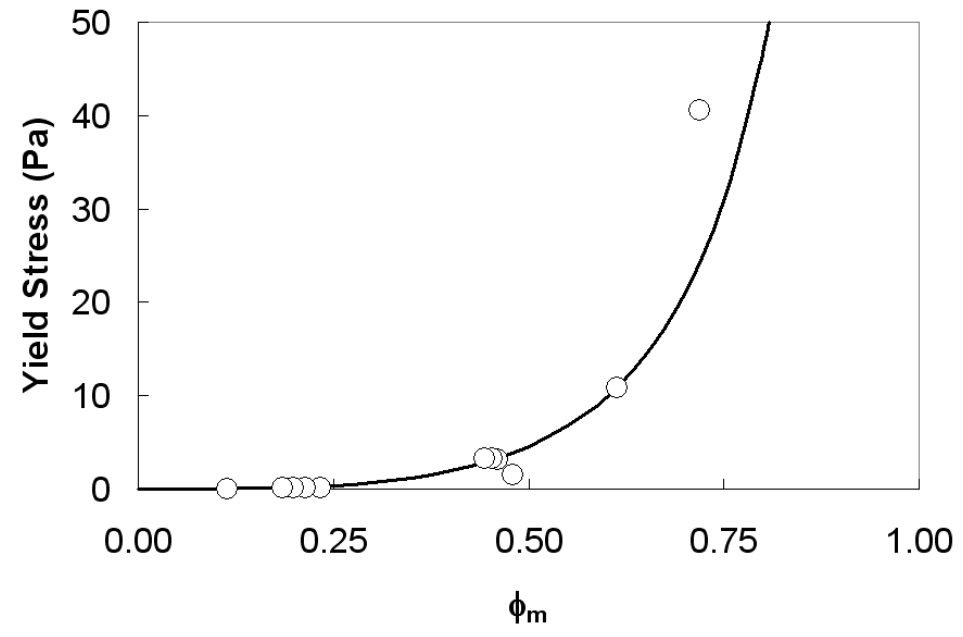

Figure 2.7. Yield stress (data) as a function of the reduced mass fraction solids. The solid line corresponds to a best-fit of Eq. (2.10).

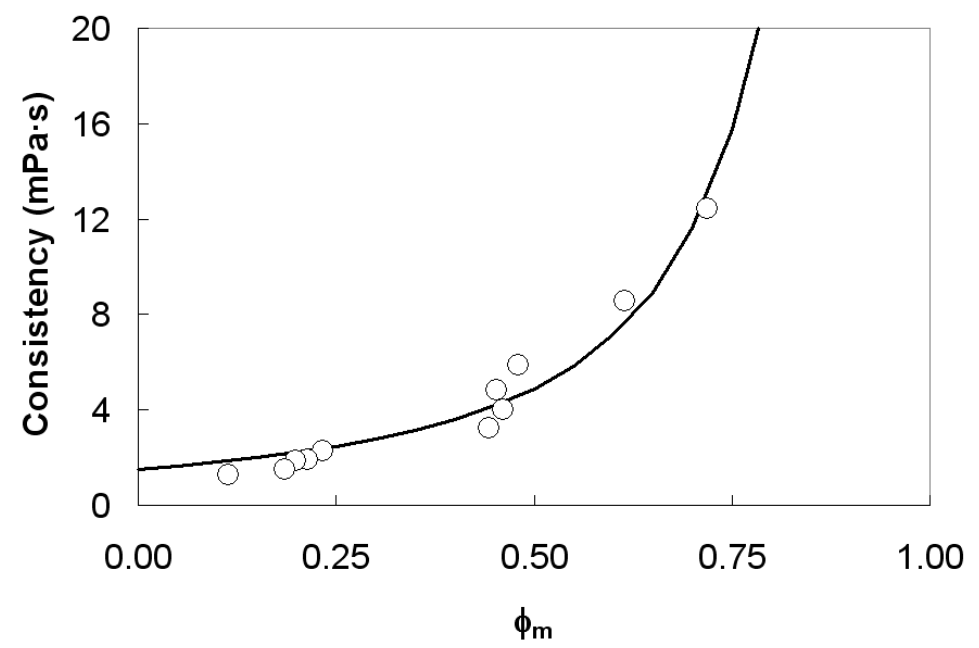

Figure 2.8. Bingham consistency (data) as a function of the reduced mass fraction solids. The solid line corresponds to a best-fit of Eq. (2.11). 
Mathematically, these correlations were developed for situations where the interstitial liquid composition remains constant. For tank retrieval, a water dilution process will be used that changes interstitial liquid composition. This limits the direct applicability of these correlations. Despite this fact, good agreement was observed between the Bingham consistency parameter and reduced solids loading correlations [Eq. (2.11)]. Since the model used to develop these correlations is similar to the Krieger-Dougherty equation which assumes non-interacting solid particles, the Bingham consistency data for Hanford TRU sludges appears to be primarily frictional based and is a weak function of dissolved species composition. Therefore, a master curve for Bingham consistency of TRU sludges has been identified as a function of moisture content. For the Bingham yield stress parameter, a relatively good fit is obtained with Eq. (2.10). Again, these empirical models are a function of reduced solids loading and do not consider inter-particle forces governed by interstitial liquid species composition. Later chapters in this report consider both friction and interparticle forces (such as sludge cohesion) with the overall purpose of improving these correlations.

\subsection{Shear Strength}

Shear strength of the TRU sludge is a significant property during tank retrieval and various process restart operations. It represents the stress required to mobilize a sedimented sludge layer. This contrasts with yield stress, which is more indicative of the yield stress observed for a sludge / slurry already in motion but approaching zero shear. The shear strength of the four tank samples were determined using a rotational rheometer equipped with a shear vane sensor system and verified against National Institute of Standards and Technology (NIST) traceable standards. The results are shown in Fig. 2.9 and indicate that the strength of the settled solids is substantially higher than indicated by the yield stress alone. Shear strength is generally observed to increase with increased solids loading. The magnitude ranges from $10 \mathrm{~Pa}$ to around $4000 \mathrm{~Pa}$. The latter indicates substantial sludge strength.

The empirical model for shear strength as a function of solids loading is similar to the yield stress

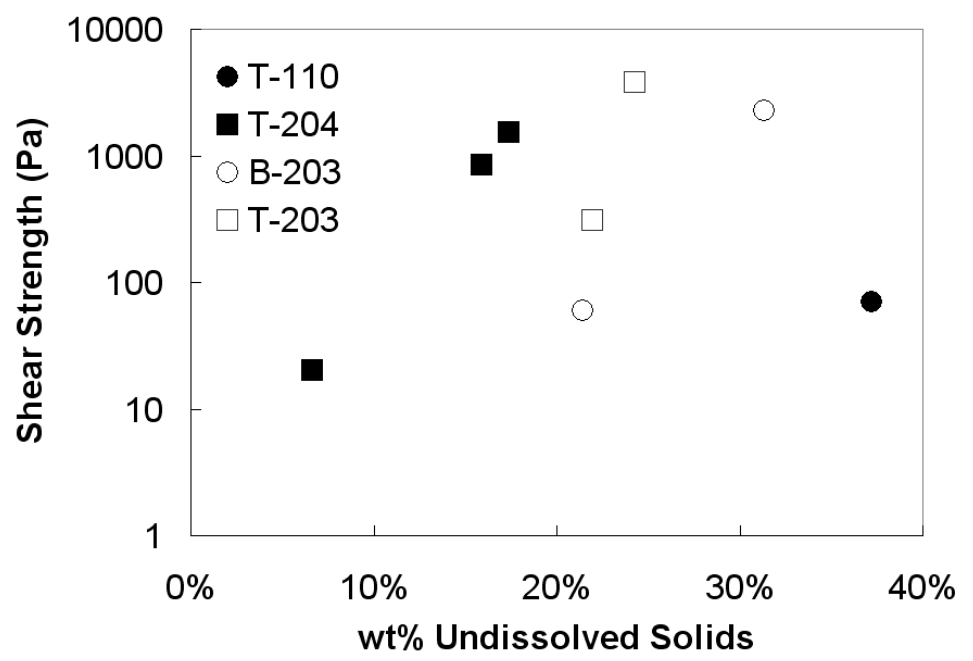

Figure 2.9. Shear strength for tank samples as a function of the mass fraction of undissolved solids. 
equation. Here, the shear strength of the material, $\tau_{v}^{\max }$ is given by,

$$
\tau_{v}^{\max }=A_{s} \frac{\phi_{m}^{3}}{1-\phi_{m}}
$$

where $A_{s}$ a constant. A best-fit of the data shown in Fig. 2.9 is presented in Fig. 2.10 It provides a relatively good fit for shear strength data of TRU sludges. These studies provide the motivation to better understand the nature of sludge shear strength. Chapter 3 presents an in-depth study regarding the development an improved model to predict shear strength given the cohesive and frictional properties of the granular solid / sludge.

\subsection{Conclusions}

This chapter presented a brief overview of sludge rheology and physical properties. The studies identify differences in the densities of materials contained in the tank waste supernatant and undissolved solids phases. The particle size distribution of the TRU tank sludges is predominantly a bimodal distribution of particles with one peak at approximately $10 \mu \mathrm{m}$ and another peak at approximately $100 \mu \mathrm{m}$. Dilution of the tank wastes with water generally results in partial dissolution of the coarse fraction. Analysis of the particle shape indicates the presence of prolate particles. Such geometries are known to cause shear-thinning effects due to particle alignment with the flow field. Chapter 6 of this report examines one potential cause for anisotropic particles in tank waste, namely deformation under ion-irradiation.

The measured rheology of the sludge is well-described by the Bingham Plastic model. Sludge yield stress and consistency coefficients, as well as the shear strength, are found to depend strongly on the reduced solids concentration. The exact dependence can be captured using correlations from the rheological literature, and the result appears to be master correlations for the Bingham consistency and yield stress of tank TRU sludges as a function of moisture content. Application of rheological models suggests that particle interactions for Hanford TRU sludges

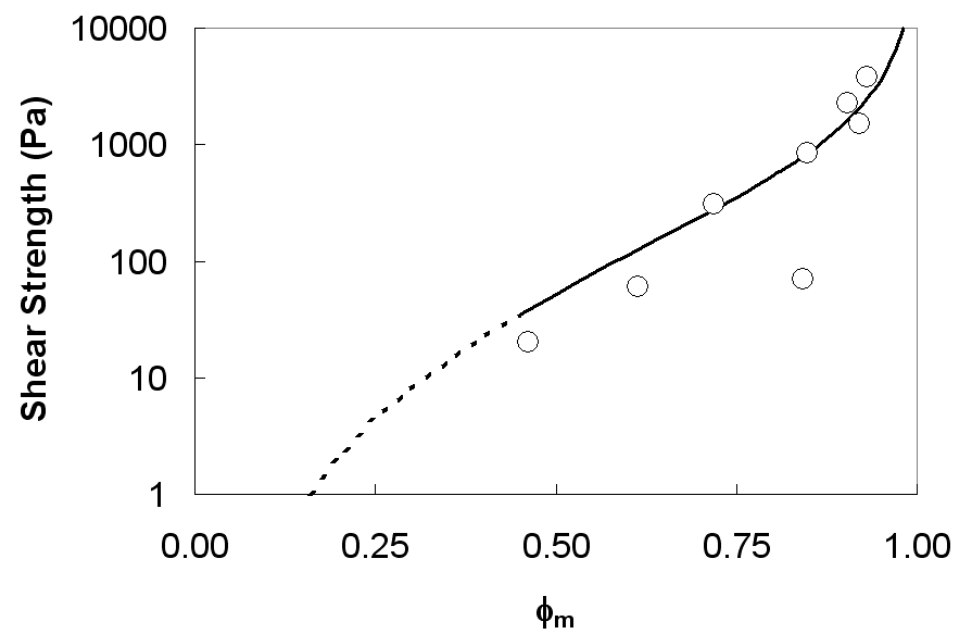

Figure 2.10. Shear strength (data) as a function of the reduced mass fraction solids. The curve corresponds to a best-fit of Eq. (2.12). The dashed portion is an extrapolation of Eq. (2.12) to concentrations lower than studied herein and represents a region of uncertainty with regard to the applicability of the fitting relationship. 
are primarily frictional and are a weak function of dissolved species composition. This observation forms the motivation for the studies of frictional and collisional rheological effects in coarse grained systems presented in Chapters 4 and 5 of this report. 



\subsection{Characterizing and Modeling Incipient Motion in Coarse Granular Slurries}

This chapter presents a method by which to characterize and model the influence of interparticle frictional and slurry / particle cohesion on the force required to initiate motion in coarse powders and slurries. The metric by which these effects is gauged is granular shear strength, which is the maximum stress that a powder or slurry can sustain during the failure of the granular matrix along a shear plane. Initial stresses in dense granular flows can be significant, as they serve to both dilate the particles and overcome static friction of contacted particles so that the bulk material may continuously deform.

The study focuses on both frictional and cohesive force contributions to a powder or slurry's shear strength. It is carried out using a rotational shear vane technique on both dry powders and powder slurries. In particular, the shear strength of 203- $\mu \mathrm{m}$ glass beads in air and slurried in water and kaolinite suspensions is determined as a function of vane immersion depth, vane geometry, and container size. The glass-bead powder / kaolinite slurry system was selected as it contains both coarse and fine fractions similar to TRU tank sludges.

Both vane immersion depth and container diameter are found to significantly impact the shear strength measured using the vane technique. An equation describing interparticle frictional and cohesive contributions to shear vane measurements is derived in an effort to correlate the experimental results to known particle and suspending phase properties along with the appropriate vertical distribution of stresses within the slurry. A Janssen stress distribution model for granular materials forms the basis for this equation and appears to explain the behavior of shear strength measurements at varying immersion depths. Rather than shear strength being a material property as is the case with flocculated colloid slurries and polymer solutions, shear strength becomes a process property where vane depth, container size, and container material can result in significant measurement variations.

While applied to only bench-scale study, the stress equations developed in this chapter incorporate the test lengths scales, and the results should allow prediction of shear strength in containers, vessels, or process lines of arbitrary size. The stress distribution model used, Janssen's equation, is well known and often used to describe the variation of pressure in engineering scale applications.

\subsection{Background}

For powders and granular slurries, a finite stress must be applied before the material will begin to flow. The stress required to transition the material from elastic deformation to viscous flow is referred to as the shear strength, and its origin can be attributed to static and kinetic friction between individual grains, strength of the matrix supporting the course fraction (i.e., the interstitial fluid), and powder/slurry cohesion arising from interparticle adhesive forces such as van der Waals and capillary forces. Incipient shear strength is an important design parameter in several fields. For soil mechanics, it can be used to gage the stability of retaining walls, hillsides and slopes (Terzaghi 1943). For powder handling in general, knowledge of shear strength can help determine whether solids will flow under the influence of gravity and allows effective design of hoppers and bins (Fayed and Otten 1997). In regards to nuclear waste remediation, several situations manifest from the Hanford Site cleanup effort in southeastern Washington State which require knowledge of a granular suspension's shear strength to properly address: 
- Nuclear waste slurries in tanks must be retrieved for treatment and disposal. These slurries are composed of precipitated metal oxide/hydroxide and sodium salt particles dispersed in a concentrated caustic solution. The slurries are contained in 177 tanks that are 23 meters in diameter with depths up to 10 meters. Particle size measurements of the contents of several of these tanks indicate a bimodal distribution of large particles on the order of $100 \mu \mathrm{m}$ dispersed in a matrix of colloids on the order of $1 \mu \mathrm{m}$. The retrieval processes often use water jets to mobilize the settled solids or sediment layer so that the diluted slurry can be removed from the tank. Jet penetration and sediment erosion rates will depend on the shear strength of the sediment (Stewart et al. 2005).

- Pulse jet mixers will be used by the Hanford Waste Treatment and Immobilization Plant (WTP). These systems consist of tubes immersed in a slurry. A periodic jet is formed through a nozzle at the end of each tube by pressurizing the tube with compressed air for discharge followed by applying a vacuum for refill. Again, if the pulse jets are shut down for a time sufficient for the solids to settle, jet penetration and the resulting mixing performance depend on the shear strength of the resulting sediment (Bamberger et al. 2005).

- Mechanical agitators will be used to mix slurries of ion exchange resin and glass former chemicals for the WTP. These materials are typically coarse grained particles on the order of several tens to hundreds of micrometers in diameter. During processing and upset conditions, the mixers may remain stationary, and a settled bed of coarse particles will need to be restarted with the agitator submerged in the sediment (Norato et al. 2000). The starting torque required to initiate movement of the mechanical mixer will depend on the shear strength of the sediment.

- Calculations of flammable gas retention and release for waste tank safety evaluation involve bubble migration models that assume the bubble rise speed is proportional to the sediment yield stress (Meyer and Wells 2000). However, in situ rheology data are available for only five tanks and rheology for the others must be derived from tests on ex situ tank waste samples. The effect of sediment depth on shear strength is not present in small waste samples, so the actual in-tank rheology is unknown in these tanks. Available in situ shear strength measurements generally indicate increasing shear strength as a function of depth (Stewart et al. 2005) consistent with this work.

Additional applications where knowledge of shear strength is important include mining and drilling (Barnes and Dzuy 2001) and food processing (Steffe 1992).

The shear strength of powders and slurries may be determined by a number of tests, including the Jenike Shear Cell (Fayed and Otten 1997), the "Slump Test" (Ancey and Jorrot 1999), and the shear vane (Liddell and Boger 1996, Ancey 2001). The latter is becoming an increasingly popular method for measuring shear strength because the vane geometry avoids slip at the surface of rotation and minimizes disruption of the sample material prior to testing. For the vane technique, the stress required to deform a fluid is determined by slowly rotating a vane immersed in a sample while continuously monitoring the resulting torque as a function of time. The shear strength is then calculated using the maximum measured torque from a torque versus time curve (see Figure 3.1) and assuming that the material is sheared only along the cylinder defined by the dimensions of the vane. In conventional vane rheometry, the stress is assumed to be distributed uniformly and isotropically over the cylindrical sheared surface (Dzuy and Boger 1985, Liddell and Boger 1996). With these assumptions, the shear strength of the material is calculated from 
Equation 3.1 (Dzuy and Boger 1985).

$$
\tau_{v}^{\max }=\frac{M_{v}^{\max }}{4 \pi R^{3}\left(\frac{H}{2 R}+\frac{1}{3}\right)}
$$

In this equation, $M_{v}^{\max }$ is the maximum measured torque on the shear vane, $\tau_{v}^{\max }$ is the shear strength of the material, $R$ is the vane radius, and $H$ is the vane height. For $\tau_{v}^{\max }$ and $M_{v}^{\max }$, the subscript $v$ refers that the shear strength is representative of the shear stress acting on the vane tool, while the superscript max refers to the fact that shear strength is determined from the maximum recorded stress. It should be noted that $\tau_{v}^{\max }$ is distinct from the yield stress value determined by interpolation to zero shear rate from a rheogram. This is because shear strength represents the stress required for the transition from a static state to viscous flow and includes frictional and gelation forces that may develop while the sample is quiescent. On the other hand, yield stress can be considered the minimum stress required to maintain viscous flow as the shear rate approaches zero. Figure 3.1 is a diagram summarizing several important dimensions and concepts of the shear vane technique.

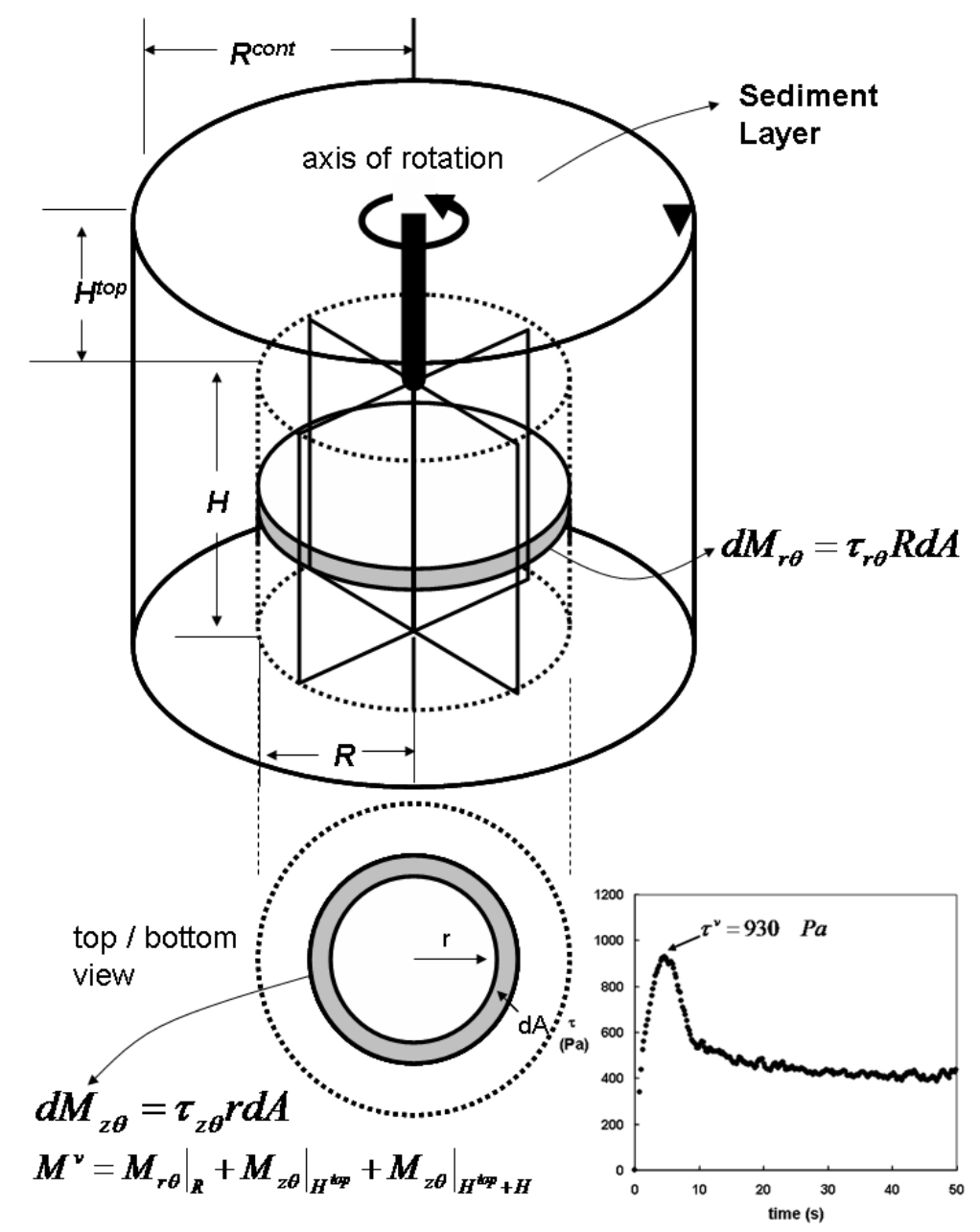

Figure 3.1. A schematic illustrating several significant dimensions and concepts for calculating shear strength using the shear vane method. The inset shows the typical output from a shear vane measurement; time is on the abscissa while shear stress calculated from Equation 3.1 is on the ordinate. 
The shear vane provides significant advantages over other tools. First, vane rotation sweeps out two vertical disk shear planes and a radial cylinder shear surface. Because both radial and vertical shear surfaces contribute to the total torque, the stress anisotropy of the granular medium can be measured indirectly. Another advantage of the shear vane tool is its similar form to mechanical agitators used to mix/re-suspend slurries, which should allow a more direct scale of up bench-scale vane experiments to engineering-scale mechanical mixing operations. One last advantage of the vane tool is its small sample footprint relative to other rheometer tools. The vane blades and impeller shaft are thin. Consequently, insertion of the vane tool does not significantly disrupt the cohesive or frictional bonds formed between the material between the blades and that surrounding the vane. In contrast, setup of more conventional tools such as the cup and rotor entail shearing the sample into a small 1-10 mm gap formed between the cup and rotor walls. Here, significant disruption of the internal structure of the samples occur prior to testing. Subsequent measurements of the "pre-sheared" material, especially those for incipient motion, may not be indicative of the true rheological behavior.

The main difficulty in measurement and prediction of granular shear strength by the vane tool is that the approach outlined above assumes an isotropic material. Specifically, the derivation of Equation 3.1 treats shear strength as constant throughout the material being tested. Although the conventional approach is constrained by suitable container size and vane immersion criteria, they serve only to minimize wall and end effects (Dzuy and Boger 1985). Unlike gelled polymer solutions and fine grained flocculated particle networks, coarse granular matter can form a continuous contact networks that transmit interparticle stress both laterally and vertically (Behringer et al. 1999). This, along with interparticle friction and lubrication, makes shear strength a process property, like hydrostatic pressure, rather than a gel-related intensive material property. As such, the shear strength of granular materials can vary with internal position and depends on the geometry of the container supporting the material. This means that results of laboratory scale shear strength measurements can vary dramatically depending on test setup / protocol and may not scale directly to process-sized equipment.

A shear vane model for characterizing / predicting granular shear strength that accounts for frictional and cohesive properties of granular matter as well as the geometric concerns would address this problem. Although no such model exists for shear vane rheometry, the literature for other rheological techniques and powder mechanics is extensive. As such, only a brief review can be given herein. Benarie (1961) discusses a method for determining powder cohesive properties of fine sands using a Couette viscometer equipped with baffled and roughened rotors. Although Benarie's approach accounts for both powder cohesion and friction, the strengths measured are done under flow conditions (i.e., low rotational rates) and incipient motion is not examined directly. Intergranular forces are treated using a linear (lithostatic) granular stress distribution independent of container geometry. Ancey and Coussot (1999) and Ancey (2001) study the effect of rotational rate and test depth for the shear vane technique on dry glass beads and glass beads lubricated by various fluids. At slow rotational rates, Ancey finds shear stress to be independent of rate and a quadratic function of vane immersion depth. Based on the test setup, where the vane tool is only partially immersed into the test slurry, this latter observations suggests a lithostatic pressure profile such that powder stress increases linearly with depth. Because Ancey and Coussot focus primarily on how the interstitial fluid lubricates and alters the shear stress as a function of flow rate, the effects of test geometry are not examined in detail with regards to vane tool shear strength determination. One article that specifically addresses the effects of system geometry on the shear strength of coarse granular material is Lee (1987). Here, Lee used a drawing-plate method to measure the shear strength of a bed of $250-297 \mu \mathrm{m}$ steel beads at various immersion depths and container sizes. He found that the shear strength of the bed increased with immersion depth to an asymptotic value that increased with the container 
diameter and that Janssen's formula (Janssen 1895) for stress distributions in granular material adequately interpreted these observations.

Few publications have examined how the shear vane impeller might be used to determine frictional and cohesive forces in dry powders and dense slurries, especially with regards to the effect of non-linear stress distributions like Janssen's formula and to incipient failure of the granular material. This chapter presents the results of recent research extending the analysis of Lee (1987) to the more conventional shear vane technique and from powder systems to slurries and sediments. In following sections, the shear vane technique is used to characterize a bed of glass beads. Both dry powder and sediments settled from slurries prepared with different interstitial liquids are examined, and the effects of vane size, vane immersion depth, container size, and interstitial fluid on sediment shear strength are investigated. A model allowing calculation of vane torque at the point of incipient motion in granular and accounting for the effects of system geometry is derived and used to interpret the experimental data presented herein.

\subsection{Methods and Materials}

Experiments were preformed to characterize the shear strength of one dry powder and two dense slurries. In all cases, Potters Industries Spheriglass ${ }^{\circledR} 1922$ Type-A glass beads were used as the coarse granular fraction. Spheriglass ${ }^{\circledR}$ Type-A beads are a nonporous soda-lime glass with a particle density of $2.5 \mathrm{~g} / \mathrm{cm}^{3}$, a dry bulk density of approximately $1.45 \mathrm{~g} / \mathrm{cm}^{3}$, and a mean particle size of $203 \mu \mathrm{m}$. For the dry powder tests, the glass beads were used "as-is". Glass/water slurries were prepared by oversaturating the glass beads with deionized water to eliminate the possibility of interparticle capillary forces. Glass/clay slurries were prepared by slowing loading the glass beads into a kaolinite matrix. The matrix was prepared by mixing deionized water with EPK Kaolin produced by Feldspar corporation to a loading of $35 w t \%$ and allowing clay-water mixture to hydrate for a period of one week. The kaolin slurry was then mixed with the glass beads in a proportion of $75 \mathrm{wt} \%$ glass bead, $25 \mathrm{wt} \%$ kaolin slurry. A second glass bead/kaolin mixture was prepared at $65 \mathrm{wt} \%$ glass bead, $35 \mathrm{wt} \%$ kaolin slurry.

The shear strength of these systems was measured using a Haake M5 viscometer fitted with fourbladed shear vanes of varying dimensions. Measurements were conducted according to the vane and container criteria outlined in Steffe (Steffe 1992). The M5 system was nominally configured to the monitor torque required for incipient motion of the granular system as the vane starts from rest and approaches a constant rotational rate. As with typical vane rheology techniques (Liddell and Boger 1996, Steffe 1992), the shear strength of granular system is associated with maximum measured torque. A data sampling rate of $5 \mathrm{~Hz}$ was used to obtain a sufficient interpolation of the torque extrema corresponding to $M_{v}^{\max }$. Experiments were performed at ambient room temperature.

A vane speed of 1/3 RPM was selected so that measurement dynamics fall in the quasi-static flow regime [i.e., where shear strength is independent of shear rate] (Liddell and Boger 1996). To confirm that the set rotational rate was in the appropriate operating region, dry glass bead shear strength was measured at rotational rates above and below 1/3 RPM. The results are presented in Table 3.1 and show the maximum torque is independent of rotational rate between 1/6 RPM and 4/3 RPM. At a rotational rate of SI7/3RPM, the measured torque significantly decreases. This may be because at higher rotational rates the data acquisition rate may not be fast enough to accurately measure the peak static torque values.

Shear strength measurements of dry glass beads were accomplished by filling a Pyrex ${ }^{\circledR}$ beaker with beads and placing the filled beaker on an adjustable laboratory stand. The stand was slowly 
Table 3.1. Measured Torque at Various Rotational Rates with $1.6 \times 1.6$ (diameter $\times$ height, $\mathrm{cm} \times \mathrm{cm}$ ) Shear Vane at Constant Depth

\begin{tabular}{cc}
\hline Rotational Rate $(R P M)$ & Measured Maximum Torque $(m N \cdot m)$ \\
\hline $1 / 6$ & $4.2 \pm 0.4$ \\
$1 / 3$ & $4.9 \pm 0.4$ \\
$2 / 3$ & $4.3 \pm 0.6$ \\
$11 / 3$ & $4.3 \pm 0.6$ \\
$21 / 3$ & $3.4 \pm 0.4$ \\
\hline
\end{tabular}

raised until the vane was immersed to the desired depth. The shear vane was rotated and the torque evolution recorded as a function of time. From this evolution, the maximum torque required to initiate powder flow is determined. After each measurement at a specific depth, the vane was removed. The glass beads were emptied into an alternate container and subsequently poured back into the original container. The purpose of this step was to verify uniform packing for each test. The above procedure was repeated by increasing vane depth incrementally until the vane could be immersed no further (because of vane shaft length). For the dry glass beads, beaker volumes of $1 \mathrm{~L}, 2 \mathrm{~L}$ and $4 \mathrm{~L}$ were used to determine dependence on container size. The radii of the beakers were measured at $5.8 \mathrm{~cm}, 7.0 \mathrm{~cm}$ and $8.6 \mathrm{~cm}$, respectively. Tests were conducted with vanes of varying dimensions $0.8 \times 1.6,1.6 \times 1.6$, and $2.2 \times 2.2$ (diameter $\times$ height [cm]) immersed at various depths.

Slurry systems were fully mixed prior to testing. The shear vane was inserted at a specific depth approximately 2 minutes after mixing to minimize settling and packing. Once the shear strength measurement was completed, the vane was removed and the test sediment re-suspended. It was found that re-suspension by pouring or rotational stirring led to structured packings in the resulting sediment that produced large measurement variations. To overcome this problem, a large spoon was used in a vertical bottom-to-top lifting motion. This "ladling" procedure effectively randomized the packing and produced consistent measurements. The $65 \mathrm{wt} \%$ glass bead, $35 \mathrm{wt} \%$ kaolin slurry was measured for one set of measurements with no remixing after the sample had remained quiescent for 20 hours to allow for settling. For these tests, a single container vane and container size, $1.6 \times 1.6 \mathrm{~cm}$ and $1 \mathrm{~L}$, respectively, are employed.

Table 3.2 summarizes the systems tested. Systems A, B, and C test the effect of container diameter; A, D, and F test the effect of vane geometry; A, F, G, and $\mathrm{H}$ test the effect of the interstitial fluid; and H, I, J test the effect of sedimentation.

\subsection{Shear Strength Model}

The surface (including both cylinder and disk portions) of rotation swept out by the tip of the rotating vane is assumed to be the slip/failure surface. Using the definition for differential torque, $d M$, at a moment arm, $r$, a force balance on a differential area of this slip surface, $d A$, can be performed:

$$
d M=\tau r d A
$$

The shear strength, $\tau$, derives from the inter-particle friction as well as adhesive and cohesive 
Table 3.2. Systems investigated as a function of vane depth.

\begin{tabular}{ccccccc}
\hline System & $\begin{array}{c}\text { Vane Radius } \\
(\mathrm{cm})\end{array}$ & $\begin{array}{c}\text { Vane Height } \\
(\mathrm{cm})\end{array}$ & $\begin{array}{c}\text { Container Radius } \\
(\mathrm{cm})\end{array}$ & $\begin{array}{c}\text { Interstitial } \\
\text { Fluid }\end{array}$ & $\begin{array}{c}\text { Volume Percent } \\
\text { Glass Beads }\end{array}$ & $\begin{array}{c}\text { Aging } \\
\text { Time }(\mathrm{min})\end{array}$ \\
\hline A & 0.8 & 1.6 & 5.8 & Air & 60 & 2 \\
B & 0.8 & 1.6 & 7.0 & Air & 60 & 2 \\
C & 0.8 & 1.6 & 8.6 & Air & 60 & 2 \\
D & 0.4 & 1.6 & 5.8 & Air & 60 & 2 \\
E & 1.1 & 1.6 & 5.8 & Air & 60 & 2 \\
F & 0.8 & 1.6 & 5.8 & Water & 60 & 2 \\
G & 0.8 & 1.6 & 5.8 & Kaolin & 0 & 2 \\
H & 0.8 & 1.6 & 5.8 & Kaolin & 60 & 2 \\
I & 0.8 & 1.6 & 5.8 & Kaolin & 50 & 1200 \\
J & 0.8 & 1.6 & 5.8 & Kaolin & 50 & 2 \\
\hline
\end{tabular}

forces. It is a complex function of the normal load and particle bed structure. Shear stress contributions from the cylinder of rotation $\left(\tau_{r \theta}\right)$ and the top/bottom disks of rotation $\left(\tau_{z \theta}\right)$ can be written by following the mathematical model for the shear strength of powders provided by Shinohara (1997):

$$
\begin{aligned}
\tau_{r \theta} & =C_{f} P_{r r}+C_{c} F \\
\tau_{z \theta} & =C_{f} P_{z z}+C_{c} F
\end{aligned}
$$

In these equations, the first and second terms represent the frictional and cohesive contributions to the shear strength, respectively. $P_{r r}$ and $P_{z z}$ are the normal stresses in the radial and vertical directions, respectively. The coefficients in these terms can be described mathematically by the microstructure and mechanics of a discrete particulate sample. Physically, Shinohara (1997) explain that $C_{f}$ corresponds to the coefficient of internal friction as the resistant effects of solid surface friction and geometrical interlocking of particles. $C_{c}$ indicates number of contact points per unit area, and $F$ represents the cohesive force between particles at a contact point. Cohesive forces can consist of a combination of Van der Waals forces, electrostatic forces, solid bridging, and liquid bridging.

When Equations 3.3 and 3.4 are substituted in Equation 3.2, the force balance on a shear vane of radius $R$ becomes:

$$
\begin{aligned}
& d M_{r \theta}=C_{f} P_{r r} R d A+C_{c} F R d A \\
& d M_{z \theta}=C_{f} P_{z z} r d A+C_{c} F r d A
\end{aligned}
$$

Of particular importance in Equations 3.5 and 3.6 is the normal loads, $P_{r r}$ and $P_{z z}$, as these anisotropic terms determine frictional forces acting between the particles. For the contained particle beds considered herein, $P_{r r}$ and $P_{z z}$ are controlled by the amount of mass of the bead bed above carried by each particle, making it a function of bed depth.

The normal load profile increases linearly with depth (similar to hydrostatic systems) under 
certain circumstances, such as for shallow or unconfined beds or beds held in wide containers. For deep beds or beds in thin containers (such as pipes or grain silos), the stress profile tends to show stress saturation. The latter phenomenon results from the contact force networks and nonuniform force networks called stress chains (Behringer et al. 1999). If stress chains propagate forces to the wall, then friction between the wall and particles allows the wall to carry part of the lithostatic load. The result is a decrease in the pressure exerted on the bottom of the container.

Because of this mechanism, the normal stress profile shows saturation with depth (Bertho et al. 2003) known as the Janssen effect (Janssen 1895, Pitman 1997, de Gennes 1999). In the current context, where a static bed of sedimented particles is being slowly sheared (and no fluidization of the bed occurs), it is reasonable to expect these intergranular forces and to include them in the stress terms in Equations 3.5 and 3.6. As will be observed in later chapters of this report, the Janssen effect is also observed under strong flow conditions. Fayed and Otten (1997) describe Janssen effects occurring during dynamic flow of coarse sands in bins and hoppers.

The intergranular pressure distribution of granular solids is often called "solid pressure" or "lithostatic pressure." The Janssen equation describes the observation that the vertical solid pressure increases linearly with depth near the free surface, but at larger depths, part of the stress is carried horizontally by the walls so that the vertical solid pressure becomes constant. Unfortunately, the Janssen equations result in inconsistencies in a local differential force balance. Much effort has been invested in resolving these inconsistencies, leading to many complex stochastic relationships and numerical simulations that are being further developed. Nevertheless, we use the Janssen stress distribution in a manner similar to Lee (1987) because of its simplicity and usefulness. In this sense, we accept the deficiencies of the Janssen relation, and we used it empirically rather than analytically.

Janssen's relation is based upon a characteristic length scale, $\lambda$.

$$
\lambda=\frac{R^{\text {cont }}}{2 \mu_{w} K}
$$

The vertical solid stress, $P_{z z}$, and horizontal solid stress, $P_{r r}$ are defined as follows:

$$
\begin{aligned}
& P_{z z}=P_{\infty}\left[1-e^{-\left(\frac{z}{\lambda}\right)}\right] \\
& P_{r r}=K P_{z z} \\
& P_{\infty}=\Delta \rho g \lambda
\end{aligned}
$$

In these equations, $z$ is the depth in the sample bed, $R^{\text {cont }}$ is the container radius, $\mu_{w}$ is the coefficient of static friction between the particles and the container wall, and $K$ equals the ratio of axial stress to radial stress (Janssen 1895, de Gennes 1999). To account for buoyant forces on the granular species, $\Delta \rho=(1-\varepsilon)\left(\rho^{p}-\rho^{s u s}\right)$. The particle density of the granular species is $\rho^{p}, \rho^{\text {sus }}$ is the density of the interstitial fluid ("suspending" phase), and $\varepsilon$ is the volume fraction of interstitial fluid. The total torque on the shear vane is calculated by integrating Equations 3.5 and 3.6 along the top, sides, and bottom surfaces of the cylinder of rotation using the Janssen pressure distribution where $P_{z z}$ is defined by Equation 3.8 and $P_{r r}$ by Equation 3.9. Summing these components produces Equation 3.11. 


$$
\begin{aligned}
M= & 2 \pi C_{f} P_{\infty} K\left[H+\lambda\left(e^{-\left(\frac{H+h}{\lambda}\right)}-e^{-\left(\frac{h}{\lambda}\right)}\right)\right] R^{2} \\
& +\frac{2 \pi C_{f} P_{\infty}}{3}\left[2-e^{-\left(\frac{H+h}{\lambda}\right)}-e^{-\left(\frac{h}{\lambda}\right)}\right] R^{3} \\
& +4 \pi C_{c} F R^{3}\left(\frac{H}{2 R}+\frac{1}{3}\right)
\end{aligned}
$$

In Equation 3.11, the vane dimensions are represented by $R$ and $H$. The immersion depth of the vane from the top of the sediment bed to the top of the vane is given by $h$. Equation 3.11 can be converted to dimensionless form by using the vane radius, $R$, as the characteristic length scale and the bulk density of the sample material, $\rho$, as the characteristic density as follows:

$$
\begin{aligned}
\hat{Y}= & 2 \pi C_{f} \hat{X}+\hat{B} \\
\hat{Y}= & \frac{M}{\rho g R^{4}} \\
\hat{X}= & K \Delta \hat{\rho} \hat{\lambda} \\
& \cdot\left[\hat{H}+\hat{\lambda}\left(e^{-\left(\frac{\hat{H}+\hat{h}}{\hat{\lambda}}\right)}-e^{\left.-\left(\frac{\hat{h}}{\lambda}\right)\right)}\right]\right. \\
& +\frac{\Delta \hat{\rho} \hat{\lambda}}{3}\left[2-e^{-\left(\frac{\hat{H}+\hat{h}}{\hat{\lambda}}\right)}-e^{\left.-\left(\frac{\hat{h}}{\hat{\lambda}}\right)\right]}\right. \\
\hat{B}= & 4 \pi \hat{F}\left(\frac{\hat{H}}{2}+\frac{1}{3}\right) \\
\Delta \hat{\rho}= & \frac{\Delta \rho}{\rho} \\
\hat{H}= & \frac{H}{R} \\
\hat{h}= & \frac{h}{R} \\
\hat{\lambda}= & \frac{\lambda}{R} \\
\hat{F}= & \frac{C_{c} F}{\rho g R}=\frac{\tau_{v}^{\max }}{\rho g R}
\end{aligned}
$$

If $\hat{X}$ is plotted against $\hat{Y}$, the data should form a line with the slope being $2 \pi C_{f}$ and the intercept, $\hat{B}$, corresponding to the interparticle forces contribution in the sample matrix, $\tau_{v}^{\max }$. Note that when the frictional contribution is negligible, the equation reduces to the usual shear vane technique equation shown as Equation 3.1 (in this case, $\tau_{v}^{\max }=C_{c} F$ ).

For the design of silo and powder systems, Fayed and Otten (1997) suggest that the values of $K$ and $\mu_{w}$ should be determined empirically by fitting experimental data subject to the following bounds: 


$$
\begin{aligned}
0.25 & \leq K \leq 0.6 \\
\left(\mu_{w, k}-0.05\right) & \leq \mu_{w} \leq\left(\mu_{w, s}+0.05\right)
\end{aligned}
$$

The coefficients of static and kinetic friction, $\mu_{w, k}$ and $\mu_{w, s}$, respectively, between glass beads were taken as 0.9 and 0.4 , respectively. These values correspond to published values of coefficients of friction between glass surfaces (Rabinowicz 1995).

\subsection{Results and Discussion}

Figure 3.2 shows torque measurements made with the vane immersed at various depths in the dry glass beads (Systems A to E), glass beads/water slurry (System F), and glass bead/kaolin slurry (Systems $\mathrm{G}$ and $\mathrm{H}$ ). In the dry glass bead systems, the measured torque increases with increasing depth, showing the role of solid pressure and fictional contacts. Additionally, the measured torque increases with vane surface area for Systems A, D, and E. The slight curvature in the torque measurements as a function of vane depth as well as the increasing torque with container size (Systems A, B, C) indicate the presence of a Janssen-form stress distribution. Torque/depth profile variations between Systems A to E indicate that experimental parameters such as vane size, container size, and measurement depth are significant for materials containing coarse particles. The effects of the interstitial fluid are investigated by the data for Systems F, G and $\mathrm{H}$. Little variation with vane immersion depth is seen for the pure kaolin slurry (System G). Janssen effects are present for slurries of beads in water and kaolin (System F and $\mathrm{H}$ ).

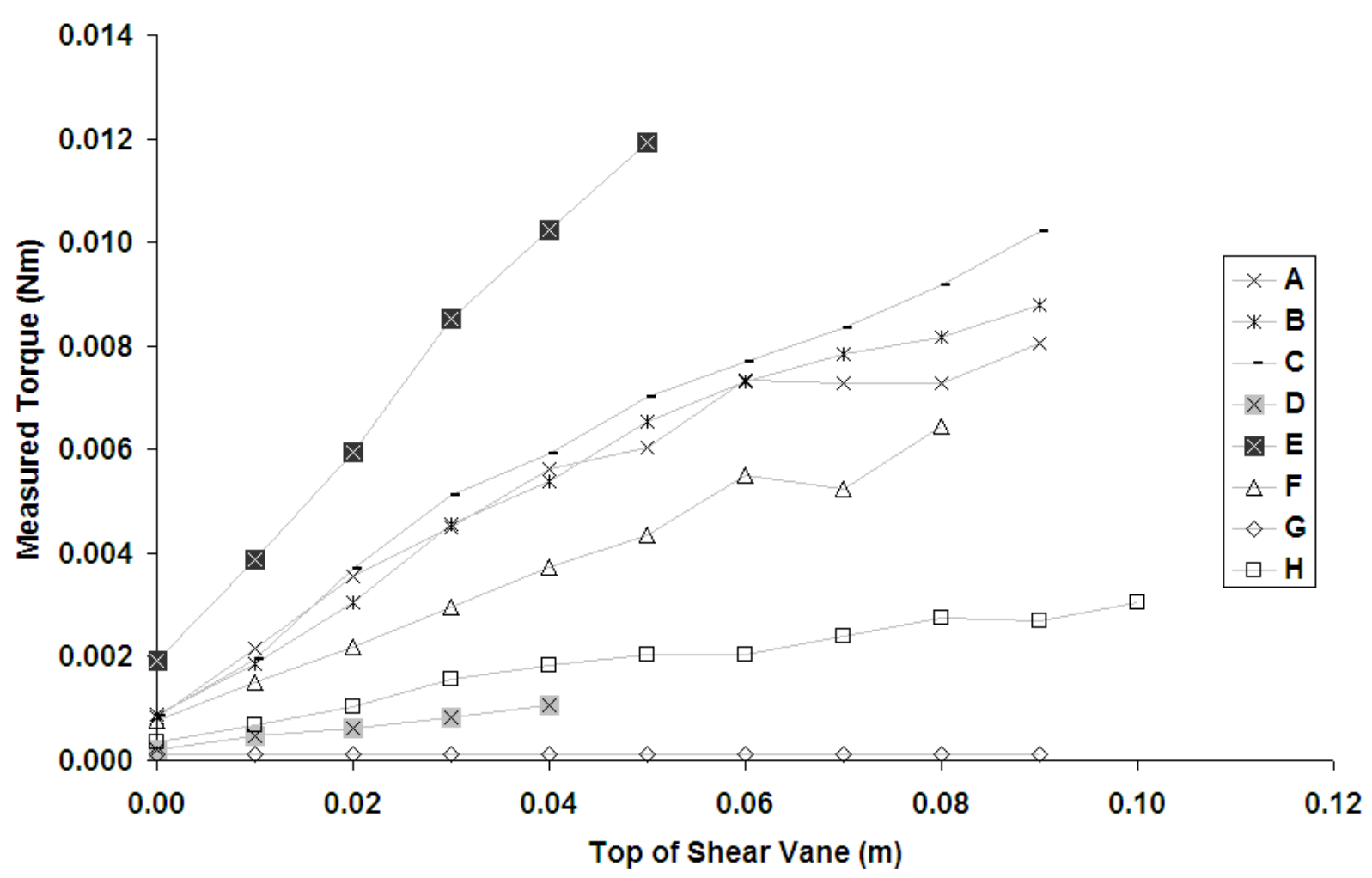

Figure 3.2. Torque measurements as a function of vane depth for the systems described in Table 3.2. 


\subsubsection{Dry Glass Bead Systems}

A constrained optimization using the bounds given by Equations 3.21 and 3.22 was performed to find the values of $K$ and $\mu_{w}$ that lead to the best linear fit for the non-dimensional shear strength data set (i.e., Equation 3.12). The optimal regression parameters are 0.39 and 0.95 for $K$ and $\mu_{w}$, respectively. The linear fit is represented as a solid line in Figure 3.3. The data collapse to a single line and indicate that effects of vane size, container size, and measurement depth can be captured by the use of the Janssen pressure distribution assumption. Table 3.3 shows the regression parameters and resulting frictional and cohesive force contributions. For Systems A-E, the frictional contribution as indicated by the parameter $C_{f}$ is relatively large whereas the cohesive contribution, indicated by $\tau_{v}^{\max }$, is small. This result is expected for granular cohesionless powders.

\subsubsection{Glass Bead Slurry Systems}

The wall friction value, $\mu_{w}$, is expected to change between powder and slurry systems because of the presence of water. Consequently, another optimization is performed for Systems F, G, and $\mathrm{H}$ constrained with the bounds given by Equations 3.21 and 3.22. The regression leads to optimal parameters of 0.43 and 0.35 for $K$ and $\mu_{w}$, respectively. As expected the coefficient of friction parameter, $\mu_{w}$, drops significantly between the wet and dry cases. Conversely, the stress distribution ratio, $K$ remains relatively constant between the two cases. The parameter $K$ reflects the anisotropy of the normal stresses in the packing and hence it is expected to be determined by the distribution and orientation of points of interparticle contact. Therefore, $K$ would be expected to

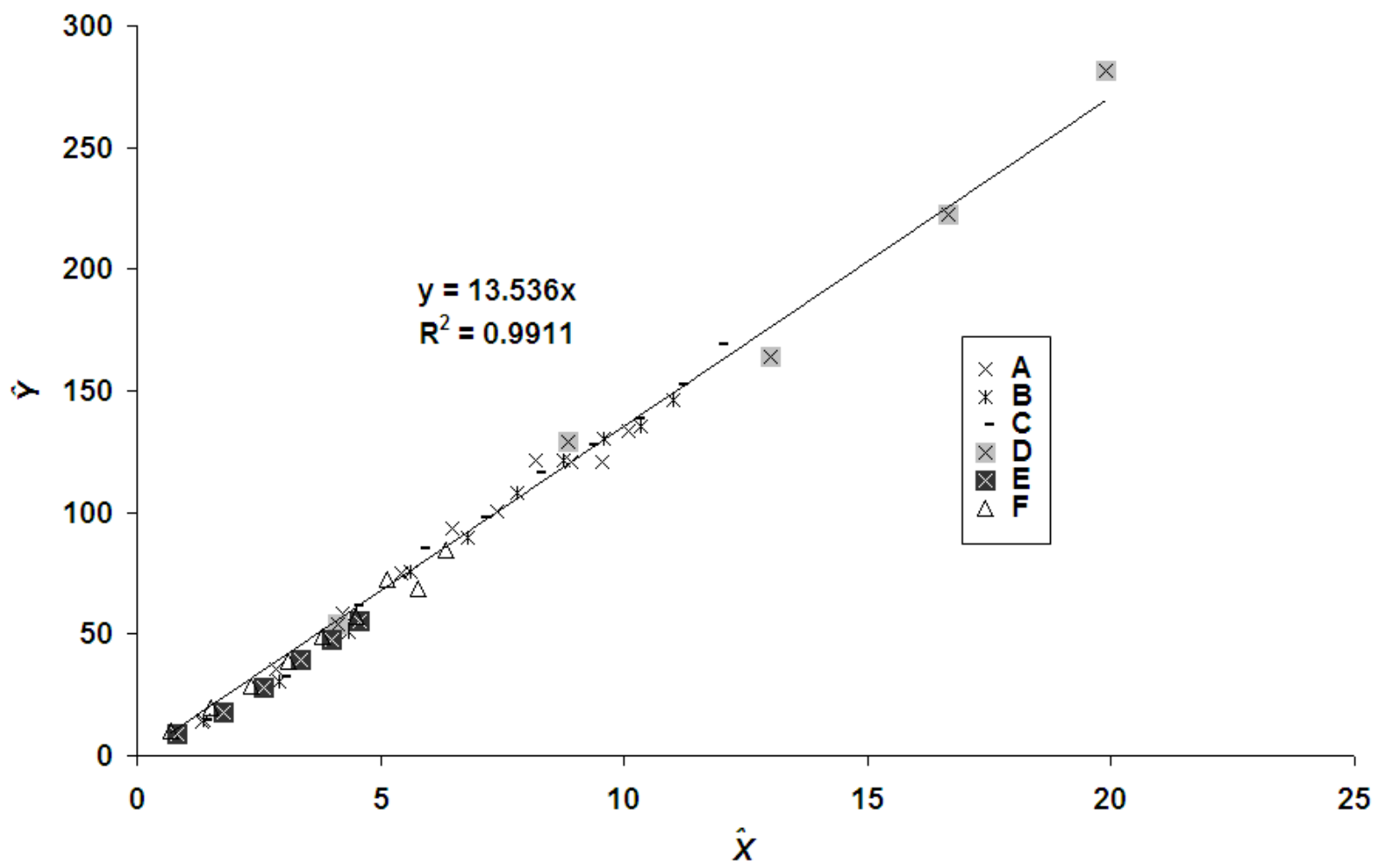

Figure 3.3. Torque measurements as a function of vane depth for Systems A-F described in Table 3.2. The data have been non-dimensionalized according to Equation 3.12 with $K=0.39$ and $\mu_{w}=0.95$ for Systems A-E. 
change when the packing arrangement changes, The presence of a liquid in the interstitial space might affect the final microstructure since it will have a role in determining how the particles settle into specific configurations. Our results show, however, that the change is not quantitatively very significant for the cases studied here. Figure 3.3 shows the non-dimensional results with water as the interstitial fluid as System F. When buoyancy effects are accounted for, these results agree well with the previous dry glass bead Systems A-E. Table 3.3 shows the regression parameters and resulting frictional and cohesive force contributions. Similar to the dry bead Systems A-E, the frictional contribution as indicated by the parameter $C_{f}$ is relatively large whereas the cohesive contribution, indicated by $\tau_{v}^{\max }$, is small.

System G consists of only the kaolin clay slurry without glass beads. As expected, this system shows a small role of frictional contacts and large cohesive force. This is reflected in the shallow slope and positive y-intercept in Figure 3.4. When the glass beads are present in the kaolin clay, both frictional contacts and cohesive forces have a role in the shear strength measurements. This is reflected by the presence of a relatively large slope and y-intercept for System H in Figure 3.4. Note that the slope or frictional contribution for the glass bead/kaolin system, H, is greater than the kaolin-only system, G, and less than the glass-bead water System, F. This may be explained by the presence of a gel structure of the interstitial kaolin in System H. In this case, the glass bead stress may distribute to the gel structure of the kaolin rather than directly through the glass bead network to the container walls. Additionally, the y-intercept for the glass bead/kaolin system, H, is in good agreement with the kaolin-only system, G. This indicates that the kaolin slurry dominates the cohesive force contribution to the shear strength of System $\mathrm{H}$.

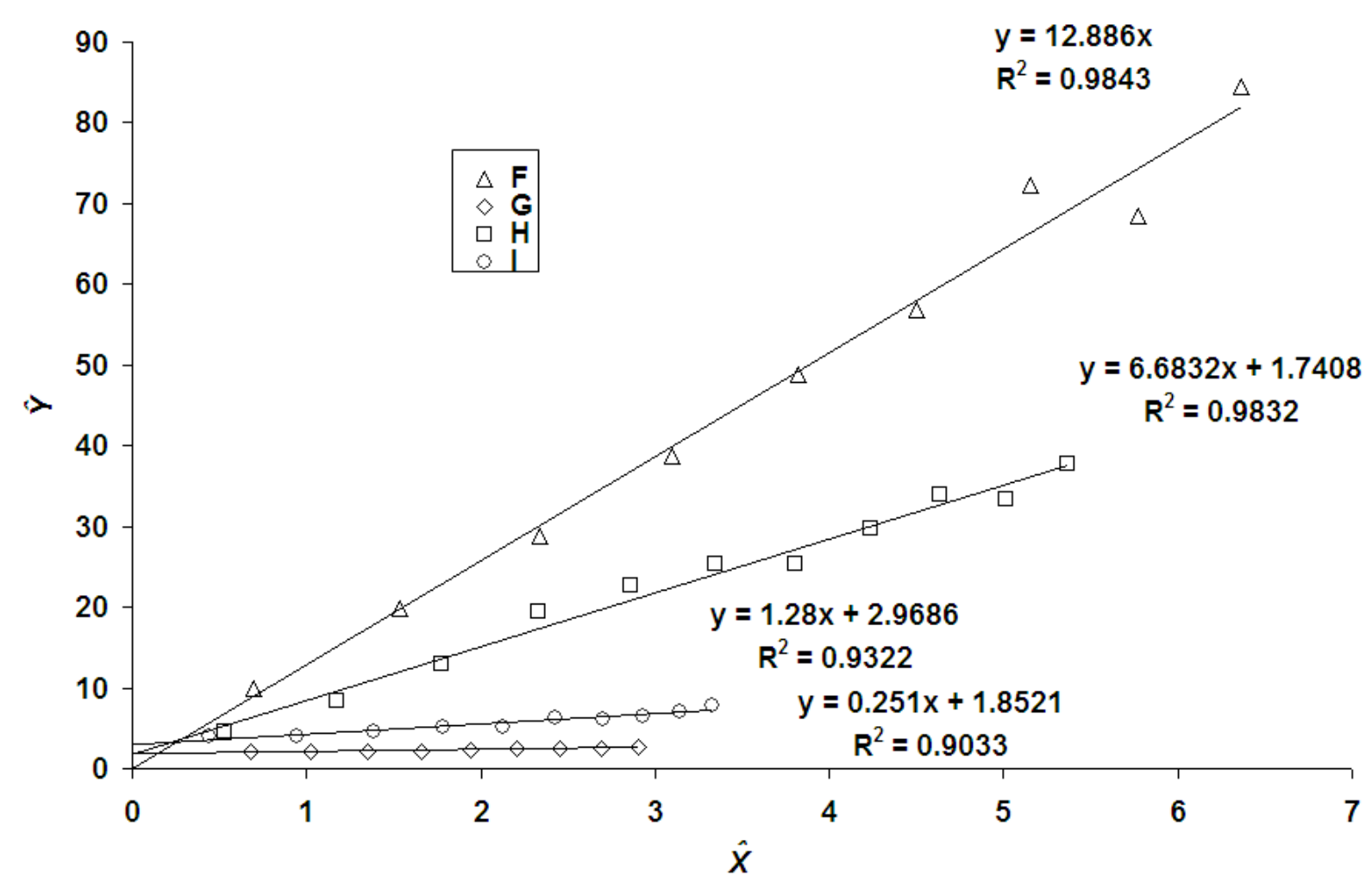

Figure 3.4. Torque measurements as a function of vane depth for Systems F-I described in Table 3.2. The data have been non-dimensionalized according to Equation 3.12 with $K=0.43$ and $\mu_{w}=0.35$. 
Interestingly, the yield stress of the kaolin clay is predicted to be sufficient to completely suspend the glass bead particles. A criterion for sedimentation of particles in a yield stress fluid is defined below.

$$
Y_{c}=\frac{\tau_{y}}{d \Delta \rho g}
$$

where $Y_{c}$ is the yield parameter, $\tau_{y}$ is the yield stress of the interstitial fluid, $d$ is the particle diameter, and $\Delta \rho$ is the density difference between the particle and the interstitial fluid. Using a force balance, Andres (1960) calculate that when $Y_{c} \geq 0.24$, the particle is stable. Beris et al. (1985) performed numerical simulations on the drag of a particle in a yield stress fluid and found the drag coefficient approaches infinity, and the particle is stable when $Y_{c} \geq 0.048$. Using the shear strength instead of the yield stress for kaolin System G shown in Table 3.3, the yield parameter results in $Y_{c}=4.6$. Consequently, the particles are expected to be completely suspended by the interstitial fluid. The yield stress was also determined using rheogram interpolation from a Haake RS600 controlled stress rheometer with a concentric cylinder geometry to be approximately 8.2 Pa. Even with this value, the yield parameter is $Y_{c}=3.4$, and particle stability is predicted.

The fact that the solid pressure phenomenon is observed in the glass bead/kaolin system indicates that sedimentation is occurring. This is consistent with visual observations of the system that show a sedimentation interface between the kaolin suspension and glass bead bed after several days of quiescence. Additionally, shear vane results from an aged suspension of glass beads in kaolin (System J; see Figure 3.5) show evidence of a settled glass bead layer. This is indicated by the nonlinearity at approximately $5 \mathrm{~cm}$ below the sample surface. Measurements taken below $5 \mathrm{~cm}$ immersion depth show evidence of solid pressures from a granular network not seen at shallower immersion depths. This nonlinearity is not seen in System I where measurements were performed immediately after the sample was mixed. System I also exhibits a profile typical of Janssen behavior, indicating the presence of particles that are not fully suspended in the kaolin slurry forming a granular stress network. Note that a larger degree of interparticle force, $\tau_{v}^{\max }$, is measured for the kaolin based System I relative to the kaolin Systems G and H shown in Table 3.3. This might be explained by a combination of experimental variation and batch-tobatch variability in Systems I, J and G,H.

Laxton and Berg (2005) discussed one explanation for this sedimentation discrepancy. In their work, Laxton and Berg (2005) observed sedimentation of colloids dispersed in a gel network that meets the yield parameter stability criterion proposed by Andres (1960). Laxton and Berg (2005) propose a second requirement for predicting the sedimentation stability of a system; the suspending medium must have $\tan \delta<1$. The phase angle of the kaolin was measured with the

Table 3.3. Linear regression results for the systems described in Table 3.2.

\begin{tabular}{ccccc}
\hline System & $K$ & $\mu_{w}$ & $C_{f}$ & $\tau_{v}^{\max }(\mathrm{Pa})$ \\
\hline A-E & 0.39 & 0.95 & 2.2 & 0 \\
F & 0.43 & 0.35 & 2.1 & 0 \\
G & 0.43 & 0.35 & 0.04 & 11 \\
H & 0.43 & 0.35 & 1.1 & 16 \\
I & 0.43 & 0.35 & 0.2 & 26 \\
\hline
\end{tabular}




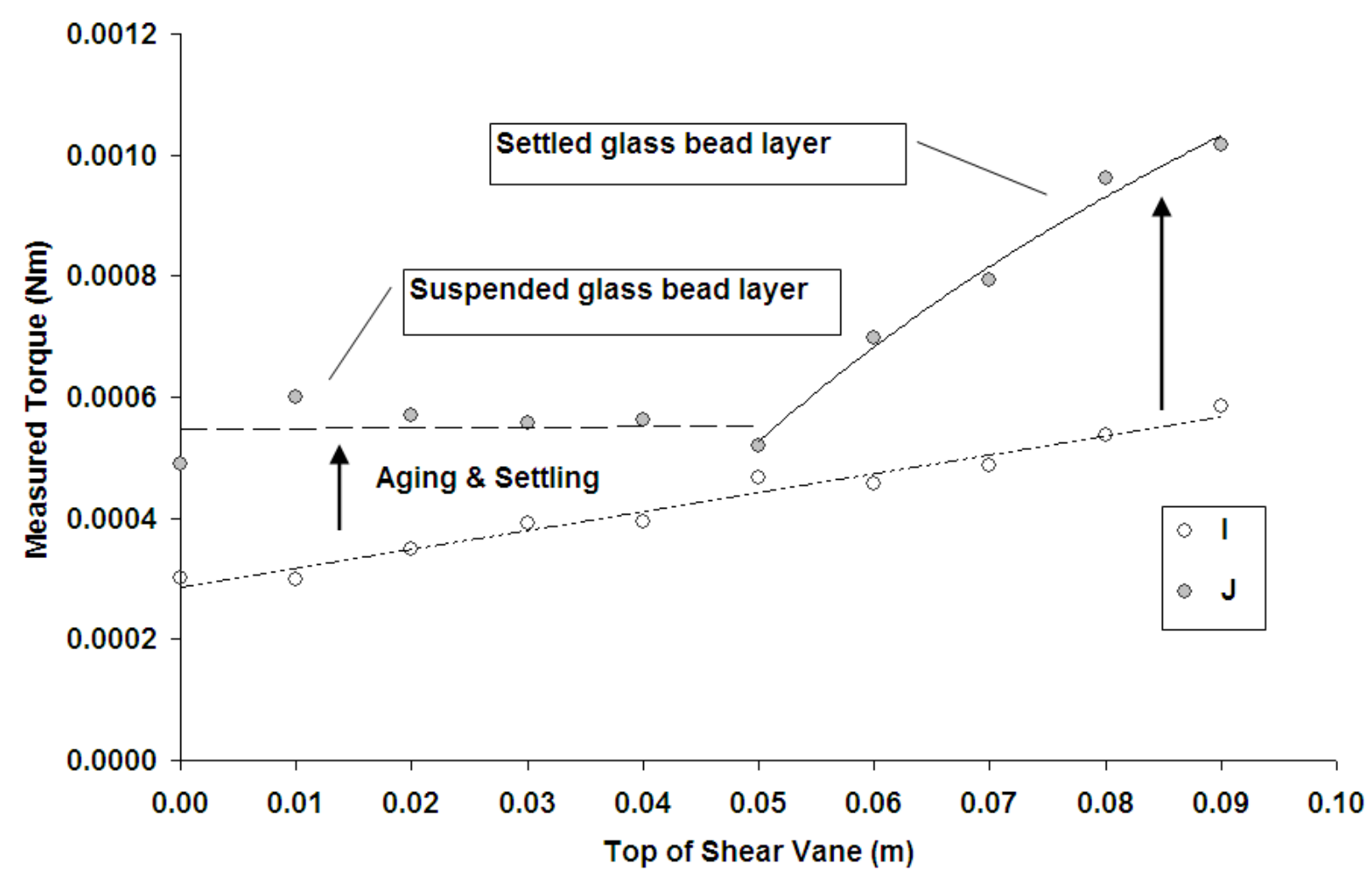

Figure 3.5. Torque measurements as a function of vane depth for System I described in Table 3.2. This system consists of a mixture of glass beads dispersed in $35 \mathrm{wt} \%$ kaolin clay slurry. The system was allowed to sediment for $1200 \mathrm{~min}$ before measuring shear strength. These results indicate a settled glass bead layer at an immersion depth of approximately $5 \mathrm{~cm}$.

Haake RS600 rheometer with a concentric cylinder geometry at an oscillation of $1 \mathrm{~Hz}$ and found to be $\tan \delta=1.5$. This indicates that viscous forces dominate elastic forces, and kaolin behaves more as a fluid than a gel. Hence, the stability criteria proposed by Laxton and Berg (2005) are not met, and the system is expected to sediment. While interesting, sedimentation of particles dispersed in a gel network is not the focus of this work. A further investigation of these stability criteria will be the focus of future work.

\subsection{Conclusions}

Results of the shear strength experiments show that immersion depth and container diameter significantly impact the measured shear strength. A model accounting for the effects of interparticle friction, slurry cohesion, vane size and immersion depth, and container geometry was derived using a Janssen stress distribution. This model was able to adequately explain experimental observations with regards to variations in container size and vane immersion depth. The functional form of the Janssen formula then provides a potential scaling relationship to apply small-scale shear vane results for various engineering applications dealing with coarse-grained slurry systems. With further refinement of the stress distribution equations in granular material to an analytical form, the vane technique may be used to quantify interparticle and frictional force contributions as well as granular packing information in the context of structural models.

The observations and theories described in this chapter bear out a number implications for the 
mobilization of TRU sludges. First, the failure strength of the material (as given by its shear strength) increases in deeper regions of the bed because of lithostatic loading. From a practical point of view, this means that the upper portions of the bed should be mobilized first to avoid strong lithostatic forces. The mobilization should be accompanied by fluidization of the particles in the upper layer. This will eliminate the continuous particle contact network and the lithostatic forces that result from it. This will lower the lithostatic load on the deeper layers and, in turn, make them easier to mobilize. The model developed in this chapter facilitates proper selection of mixing depths and evaluation of staged mixing events.

The second implication for the mobilization of TRU sludges has to do with the existence of stress saturation effects in slurry systems. While the Janssen effect tends to limit the lithostatic loading achieved in deep beds, it can also lead to increased difficulty in removing slurry plugs from pipes. Here, the fluid force acting on a slurry plug is transmitted to the pipe wall, reducing the ultimate force that can be applied to the plug. The model and measuring techniques developed herein could be used to characterize and infer stress profiles existing in TRU sludge pipeline plugs. Strategies for mechanically dislodging the plug (as opposed to chemical treatments) could be developed based on the inferred plug stress profile. 



\subsection{A Continuum Constitutive Model for Cohesionless Granular Flows}

The previous chapter examined stresses that exist during the incipient motion in granular solids. In this chapter, a constitutive model is developed to describe shear flows of cohesionless granular solids. The model is based on the postulate that the friction coefficient and the solids fraction in a moving granular material are exclusive functions of the inertial number, which represents the ratio of inertial to normal stress forces. The constitutive equation obtained has the same form as a multidimensional Bingham fluid model, albeit with apparent viscosity and yield stress that depend on the vertical normal stress. The model is applied to previously published experimental results dealing with three-dimensional shear flows of granular beds made up of cohesionless spherical particles. The first case analyzed corresponds to a granular bed moving on top of a rotating disk. The model captures the main trends of the velocity profiles with a single adjustable parameter. The second case is a conventional Couette flow, for which the model is capable of representing the velocity and solids fraction profiles measured experimentally.

\subsection{Background}

As stated in the introductory chapter of this report, course granular powders and slurries exhibit highly non-Newtonian behavior because of frictional and collisional interactions between individual grains. In the previous chapter, the role of interparticle friction and slurry cohesion was shown to have a profound impact on the force required to mobilize granular material. Once the powder or slurry is mobilized, the effect of particle collisions on the granular rheology must also be considered.

For a mobilized cohesionless granular material, three basic flow regimes have been observed: quasi-static, intermediate, and kinetic. Slow or poorly fluidized flows are quasi-static and share many of the characteristics of incipient motion stress dynamics. Specifically, frictional interactions dominate and shear stress is independent of shear rate. Fast or highly fluidized flows are dominated by particle collisions, and show similar dynamics to ideal gases. Molecular kinetics theories have been developed to describe kinetic flows dynamics and energy dissipation (Campbell 1990, Goldhirsch 2003, Jenkins and Richman 1985). Intermediate flows exist between these two extremes. Here, both interparticle friction and particle collisions influence the flow dynamics. Intermediate flows are still an extensive area of research. The recent works of da Cruz et al. (2005) and Jop et al. (2006) have developed relationships that correlate powder properties, such as apparent bulk friction and solids fraction, to the local rate of deformation and powder fluidization.

The intermediate region is of importance, as flow of granular materials can often span at least two of the flow regimes outlined above. For example, rheometric flows of granular materials are characterized by the formation of well-defined shear bands that resemble qualitatively the behavior of a fluid with a yield stress. These flows may fall in an intermediate region between the frictional and collisional flow regimes, while possessing boundaries of unyielded material in which the applied stress does not overcome bulk granular friction. The constitutive behavior of granular matter in this type of flow has been the subject of recent attention [e.g. MiDi (2004), Depken et al. (2006), Tardos et al. (2003), da Cruz et al. (2005), Jop et al. (2006),]. da Cruz et al. (2005) proposed a constitutive model based on the discrete simulation of two-dimensional simple shear of a dense ensemble of disks in the absence of gravity. Their results confirm a proposal put forth by previous works [Savage and Hutter (1989), Ancey et al. (1999)] that establishes that the flow regime and rheological parameters scale with a dimensionless number that represents the relative 
strength of inertia forces with respect to the confining pressure. For a three-dimensional granular medium made up of monodisperse spheres undergoing simple shear flow at a shear rate $\dot{\gamma}_{0}$, this dimensionless number, termed inertial number, is defined as

$$
I=d \sqrt{\frac{\rho_{p}}{P}} \dot{\gamma}_{0}
$$

where $d$ is the particle diameter, $\rho_{p}$ is the particle density, and $P$ is local pressure. The approach followed by da Cruz et al. is based on the postulate that there are two basic constitutive relations that a granular material obeys: 1) a friction law, by which the macroscopic coefficient of friction, defined as

$$
\mu^{*}=S / P
$$

where $S$ is the local shear stress, depends only on $I$; and 2) a dilatancy law, by which the volume fraction of solids in the medium, $v$, depends also exclusively on $I$.

In their 2-D simulations, da Cruz et al. find that the effective friction coefficient depends linearly on $I$ for $I<0.20$,

$$
\mu^{*}=\mu_{\min }^{*}+b I
$$

and that it tends to achieve a constant value at higher values of $I$. In the same range, they also found that the solids fraction depends linearly on $I$,

$$
\mathrm{v}=\mathrm{v}_{\max }-a I
$$

These constitutive laws bridge the gap between the frictional and collisional flow regimes, allowing for a description of granular rheology over a wide range of conditions.

More recently, Jop et al. (2006) proposed a constitutive model based on the three-dimensional constitutive equation used to represent viscoplastic behavior (such as Bingham fluids),

$$
\sigma_{i j}=-P \delta_{i j}+\mu_{B} \dot{\gamma}_{i j}
$$

where $\sigma_{i j}$ and $\dot{\gamma}_{i j}$ are components of the total stress and rate of strain tensors, respectively; and the apparent viscosity was taken to be a function of the inertial number and the pressure as fol- 
lows,

$$
\mu_{B}=\frac{\mu(I) P}{\dot{\gamma}}
$$

In this equation, $\dot{\gamma}=\sqrt{0.5 \dot{\gamma}_{i j} \dot{\gamma}_{i j}}$ is the second invariant of the rate of strain tensor. Jop et al. were successful in representing experimental data for flow over an inclined surface using an $a d$ hoc representation for the function $\mu(I)$.

In light of the developments in the recent literature, it is now possible to approach a variety of problems concerning flow of granular matter. Two problems of interest, and the focus of this chapter, are that of a rotating cylinder immersed in a granular material and that of rotating disk upon which a granular material rests. Despite the rich information available in the literature, current continuum formulations do not allow treatment of stress anisotropy [i.e., they assume uniform stress profiles as in Eq. (4.5)] and solids fraction changes with shear. To this end, a constitutive equation that is consistent with the frictional and dilatancy relations proposed by da Cruz et al. [equations (4.3) and (4.4)] is proposed. The resulting model captures the viscoplastic behavior represented by equation (4.5). This constitutive model is used to describe a rotational Couette-type flow and flow over a rotating disk. Results are compared against available literature data from flow studies of these geometries performed by Mueth et al. (2000) and Cheng et al. (2006).

\subsection{Constitutive Model}

The manipulation of equations (4.1) to (4.4) leads to the following equations relating the pressure and shear stress to the shear rate in one-dimensional, simple shear flows,

$$
\begin{gathered}
P=\frac{\rho_{p} a^{2} d^{2}}{\left(v_{\max }-v\right)^{2}} \dot{\gamma}_{0}^{2} \\
S=\mu_{\text {min }}^{*} P+b d \sqrt{\rho_{p} P} \dot{\gamma}_{0}
\end{gathered}
$$

It should be noted that, as $\dot{\gamma}_{0} \rightarrow 0$ (and, consequently, $I \rightarrow 0$ ), the Coulomb constitutive equation is recovered in equation (4.8) (i.e., shear stress directly proportional to pressure), while equation (4.7) yields $v \rightarrow v_{\max }$.

To generalize these equations to three dimensions, a constitutive equation that takes into account the anisotropy of static normal stresses is proposed:

$$
\sigma_{i j}=-P_{i j}+\tau_{i j}
$$

Here $P_{i j}$ is a diagonal tensor that, yields the three components of static normal stresses in the absence of motion. The vertical $(z)$ component of these static normal stresses is the confining 
pressure, $P_{z z}=P$. The deviatory stresses, $\tau_{i j}$, will be represented by a viscoplastic, Binghamtype equation that simplifies to equation (4.8) in the case of one-dimensional simple shear flow:

$$
\tau_{i j}=\mu_{B} \dot{\gamma}_{i j}
$$

where the non-Newtonian viscosity depends on the second invariant of the rate of strain tensor by

$$
\mu_{B}=\frac{m u_{m i n}^{*} P}{\dot{\gamma}}+b d \sqrt{\rho_{p} P}
$$

The first term of this Bingham viscosity represents the yield stress of the material, which gives a nonzero contribution to the shear components of the stress tensor under static conditions, representative of frictional forces between particles.

The constitutive model is completed by using equation (4.7) (replacing $\dot{\gamma}_{0}$ by $\dot{\gamma}$ ), which can be interpreted as the equation that determines the solids fraction (dilatancy law).

The present analysis will be restricted to flows in which 1) the granular material does not move in the vertical direction ( $z$ ), and 2) the material has a flat free surface at $z=0$, on which no stresses are applied. Assuming that the solids fraction does not experience significant variations in the $z$ direction, the pressure distribution in the granular material under static (or quasi-static) conditions follows a Janssen type equation [Poloski et al. (2006), Bertho et al. (2003)],

$$
P=v \rho_{p} g \lambda\left(1-e^{-z / \lambda}\right)
$$

where $g$ is acceleration of gravity and $\lambda$ is the Janssen length measured under dynamic conditions. If the analysis is confined to a depth $z=H_{b}$ such that $H_{b}<<\lambda$, equation (4.12) simplifies to a lithostatic profile,

$$
P=v \rho_{p} g z
$$

If variations of $v$ with vertical position are significant, the pressure would be determined by

$$
P=\rho_{p} g \int \mathrm{v} d z_{0}^{z}
$$

\subsection{Flow over a Rotating Disk}

Cheng et al. (2006) presented studies of a rotating disk system where a granular material contained in a cylinder of radius $R_{0}$ and height $H_{b}$ is sheared by rotating a portion of the bottom of 
the container (radius $R$ ) at a constant angular velocity, $\Omega$ (Figure 4.1). Materials employed were packed beds of approximately monodisperse particles that were cohesionless. Detailed measurements of the angular velocity profiles were provided. A mathematical description of this system will be formulated using the constitutive model proposed above.

It is assumed that the only non-zero component of the velocity field is $v_{\theta}=v_{\theta}(r, z)$. In addition, the pressure distribution is treated as lithostatic [equation (4.13)], and variations of $v$ within the medium are assumed negligible with respect to the undisturbed value for the packed beds. The linear momentum balance in the $\theta$ direction simplifies to

$$
\frac{1}{r^{2}} \frac{\partial\left(r^{2} \tau_{r \theta}\right)}{\partial r}+\frac{\partial \tau_{z \theta}}{\partial z}=0
$$

The constitutive model presented above will be generalized using the non-Newtonian viscosity defined by Eq. (4.11). Within this framework, the shear stresses can be expressed in terms of the appropriate component of the deformation rate tensor as follows,

$$
\tau_{r \theta}=r \mu_{B} \frac{\partial\left(v_{\theta} / r\right)}{\partial r}
$$

$$
\tau_{z \theta}=\mu_{B} \frac{\partial v_{\theta}}{\partial z}
$$

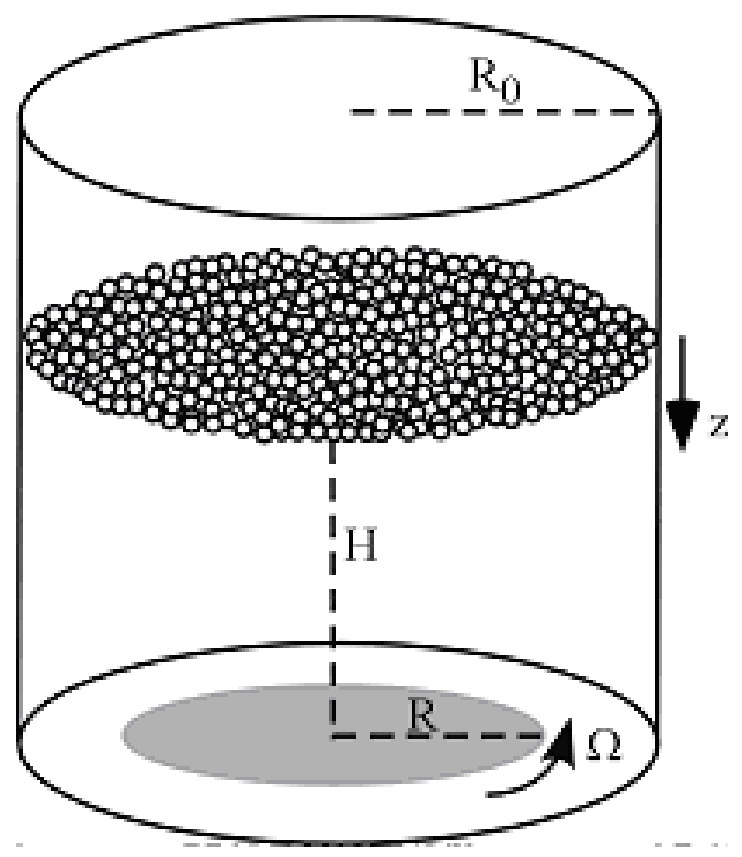

Figure 4.1. Geometry of the rotating disk system. 
Combination of equations (4.15) to (4.17) leads to the following governing equation for $v_{\theta}$,

$$
\frac{1}{r^{2}} \frac{\partial}{\partial r}\left[r^{3} \mu_{B} \frac{\partial\left(v_{\theta} / r\right)}{\partial r}\right]+\frac{\partial}{\partial z}\left(\mu_{B} \frac{\partial v_{\theta}}{\partial z}\right)=0
$$

This partial differential equation is subject to the following boundary conditions

$$
\begin{aligned}
& \frac{\partial v_{\theta}}{d z}=0, \text { at } z=0 \\
& v_{\theta}=\Omega r, \text { at } z=H_{b} \\
& v_{\theta}=0, \text { at } r=0 \\
& v_{\theta}=0, \text { at } r=R_{y}(z)
\end{aligned}
$$

where $R_{y}$ represents the radial position at which both components of the shear stress fall below the yield stress (yield surface). It is assumed that $R_{y}$ is always below $R_{0}$, so that there is always an external region of unyielded material.

The non-Newtonian viscosity [equation (4.11)] is related to the local shear rate, determined by

$$
\dot{\gamma}=\sqrt{\left[r \frac{\partial\left(v_{\theta} / r\right)}{\partial r}\right]^{2}+\left(\frac{\partial v_{\theta}}{\partial z}\right)^{2}}
$$

To limit the parameter space encompassed by the model, the equations were made dimensionless using the following definitions: $\hat{\omega}=v_{\theta} /(\Omega r), \zeta=r / R, \eta=z / R,{ }^{\prime}=\mu_{B} /\left(\rho_{p} b d \sqrt{v g R}\right)$. The problem is formulated in dimensionless form as follows,

$$
\begin{gathered}
\frac{1}{\zeta^{3}} \frac{\partial}{\partial \zeta}\left[\zeta^{3} \mu^{\prime} \frac{\partial \hat{\omega}}{\partial \zeta}\right]+\frac{\partial}{\partial \eta}\left(\mu^{\prime} \frac{\partial \hat{\omega}}{\partial \eta}\right)=0 \\
\frac{\partial \hat{\omega}}{\partial \eta}=0, \text { at } \eta=0
\end{gathered}
$$




$$
\begin{aligned}
& \hat{\omega}=1, \text { at } \eta=\alpha \\
& \frac{\partial \hat{\omega}}{\partial \zeta}=0, \text { at } \zeta=0 \\
& \hat{\omega}=0, \text { at } \zeta=\zeta_{y}
\end{aligned}
$$

where $\alpha=H_{b} / R$, and the dimensionless non-Newtonian viscosity is

$$
\mu^{\prime}=\frac{\beta \eta}{\zeta \Gamma}+\eta
$$

The dimensionless shear rate is given by

$$
\Gamma=\sqrt{\left(\frac{\partial \hat{\omega}}{\partial \zeta}\right)^{2}+\left(\frac{\partial \hat{\omega}}{\partial \eta}\right)^{2}}
$$

and

$$
\beta=\frac{\mu_{\min }^{*} \sqrt{v g R}}{b d \Omega}
$$

The dimensionless location of the yield surface, $\zeta_{y}(\eta)$ is part of the problem solution. Hence, the previous formulation contains only two parameters: $\alpha$ and $\beta$.

The model discussed above is compared against experimental data reported by Cheng et al. (2006). Their experimental setup consisted of two different cylindrical cells with $R_{0}=43.9 \mathrm{~mm}$ and $72.25 \mathrm{~mm}$ with rotating disks at the bottom with $R=32.5 \mathrm{~mm}$ and $55.5 \mathrm{~mm}$, respectively. The larger cell was used to measure surface velocities using high-speed video with a packing of mustard seeds $(d=1.9 \mathrm{~mm})$, with $\Omega=1.96 \mathrm{rad} / \mathrm{s}$. The smaller cell was filled with a mixture of poppy and rajagara seeds $(d=0.85 \mathrm{~mm}), \Omega=1.01 \mathrm{rad} / \mathrm{s}$, and angular velocity profiles at various axial positions were measured using MRI. No vertical motion of the material was reported. Experiments were conducted at various bed depths. Deeper beds showed an evolution of the profiles from a constant angular velocity at the surface of the rotating disk to an almost stationary bed at the free surface.

Angular velocity profiles corresponding to the results of Cheng et al. (2006) are calculated by numerically solving equations (4.24) to (4.28) using a finite difference algorithm with GaussSeidel iteration. The starting point for the iterative procedure was the solution of the problem 
for a Newtonian fluid with a zero velocity condition at $\zeta=\zeta_{y}=1$, which was obtained by letting $\mu^{\prime}=1$ in the formulation (an analytical solution was obtained by separation of variables to generate these profiles).

The best fits of the experimental angular velocity profiles obtained for a deep bed $(\alpha=0.88)$ by varying $\beta$ [equation (4.31)], which is the only adjustable parameter in the model, are shown in Figure 4.2. The model developed in this chapter is capable of adequately representing the behavior of the profiles at various depths using a single value for $\beta$. The yield surface (as determined from the calculated profiles) is very close to the edge of the rotating disk $\left(\zeta_{y} \rightarrow 1+\right)$ for $\eta>0.7$ (Figure 4.2), and moves inward as the free surface is approached. The discrete microscopic model presented by Cheng et al. (dashed lines in Figure 4.2) cannot reproduce the relatively high speeds measured in deep regions of the bed.

To give an idea about the sensitivity of the model to the fitting parameter $\beta$, Figure 4.3 shows the shape of the profiles obtained for various limiting conditions. The limit $\beta \rightarrow \infty$ represents a Coulomb solid. In this case, at low depths, the material tends to move as a solid block, with a very steep velocity profile in the vicinity of the yield surface. The limit $\beta=0$ corresponds to

$\omega$

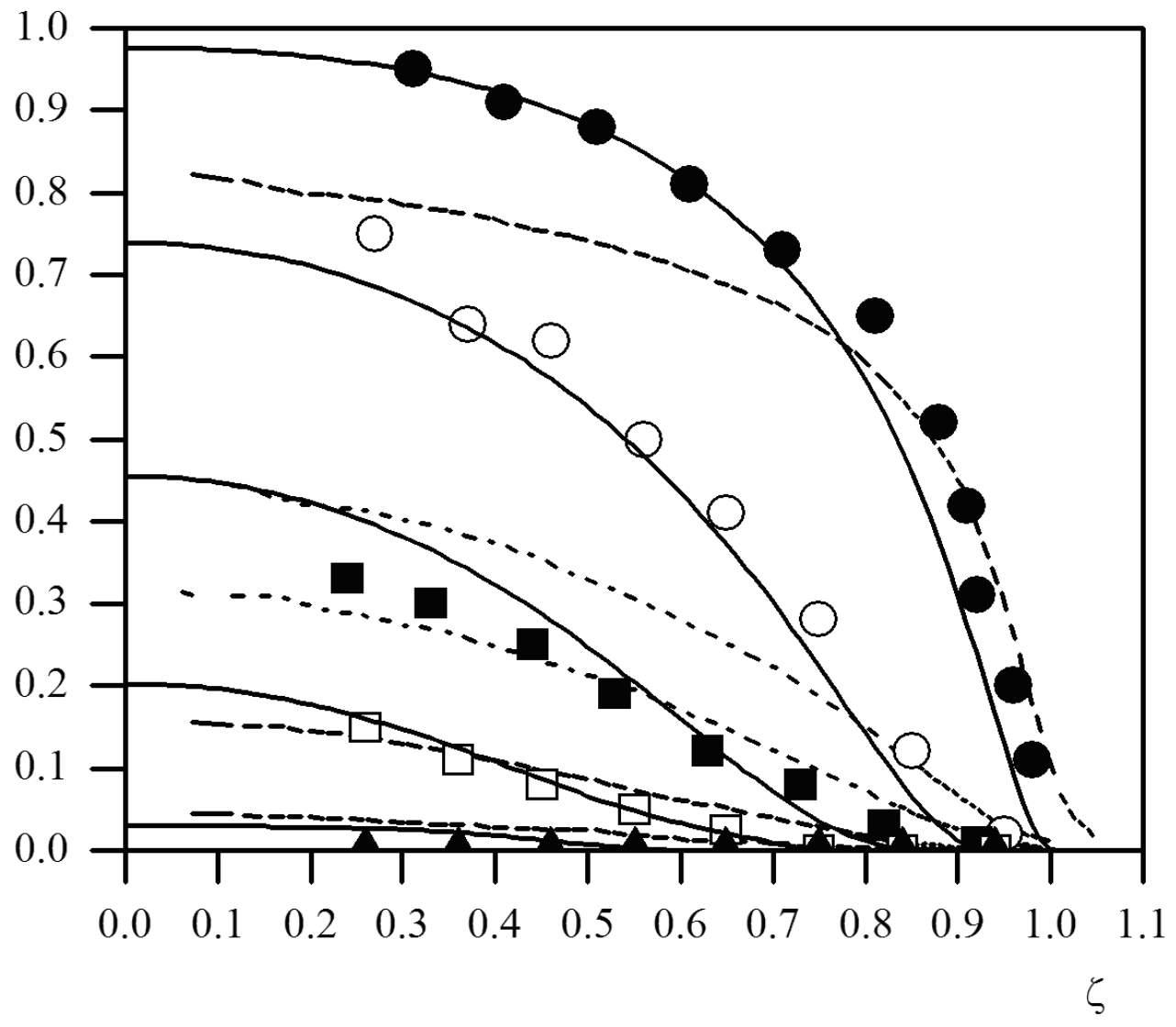

Figure 4.2. Experimental (symbols) [Cheng et al. (2006)] and fitted (solid lines) angular velocity profiles for rotating disk flow in a bed with $\alpha=0.88$. The symbols and accompanying lines correspond to: $\eta=0.792(\bullet), \eta=0.6952(\circ), \eta=0.6072(\mathbf{\square})$, $\eta=0.5104(\square), \eta=0.2376(\Delta)$. The solid lines represent the best fit to the whole set of data, obtained for $\beta=50$. The dashed lines are results of a discrete simulation (details in Cheng et al. (2006), and references therein). 
a fluid without yield stress but with a viscosity that varies with depth. This case and the Newtonian fluid are incapable of representing the observed axial and radial changes of the angular velocity.

Since the parameter beta is independent of the bed depth, the same value $(\beta=50)$ was used to predict the velocity profiles for $\alpha=0.4$. The results are shown in Figure 4.4. Clearly, the model captures the behavior of the shallow bed. For the axial positions reported in Figure 4.4, the calculated yield surface is located in the interval $1<\zeta_{y}<1.2$. In this case, a sizable portion of the bed rotates with the disk's angular velocity.

Cheng et al. (2006) also performed experiments in which the surface angular velocity was measured. As stated above, these experiments were done in a different cell and at different conditions from the velocity profile experiments. The values of $R, d$ and $\Omega$ are different for the two sets of experiments. Assuming that the parameter $b$ is invariant, the fitted value $\beta=50$ from Figure 4.2 can be used to predict $\beta=15$ for the surface flow experiments. Figure 4.5 shows a comparison between the model's predictions and the experimental data. At low $\alpha$ 's (shallow beds), the free surface angular velocity at the axis of rotation $\left(\hat{\omega}_{0}\right)$ is equal to the disk angular velocity. As the bed becomes deeper, $\hat{\omega}_{0}$ drops with a stronger dependence on the bed depth than for a Newtonian fluid. From Figure 4.5 it can be seen that the model predictions capture

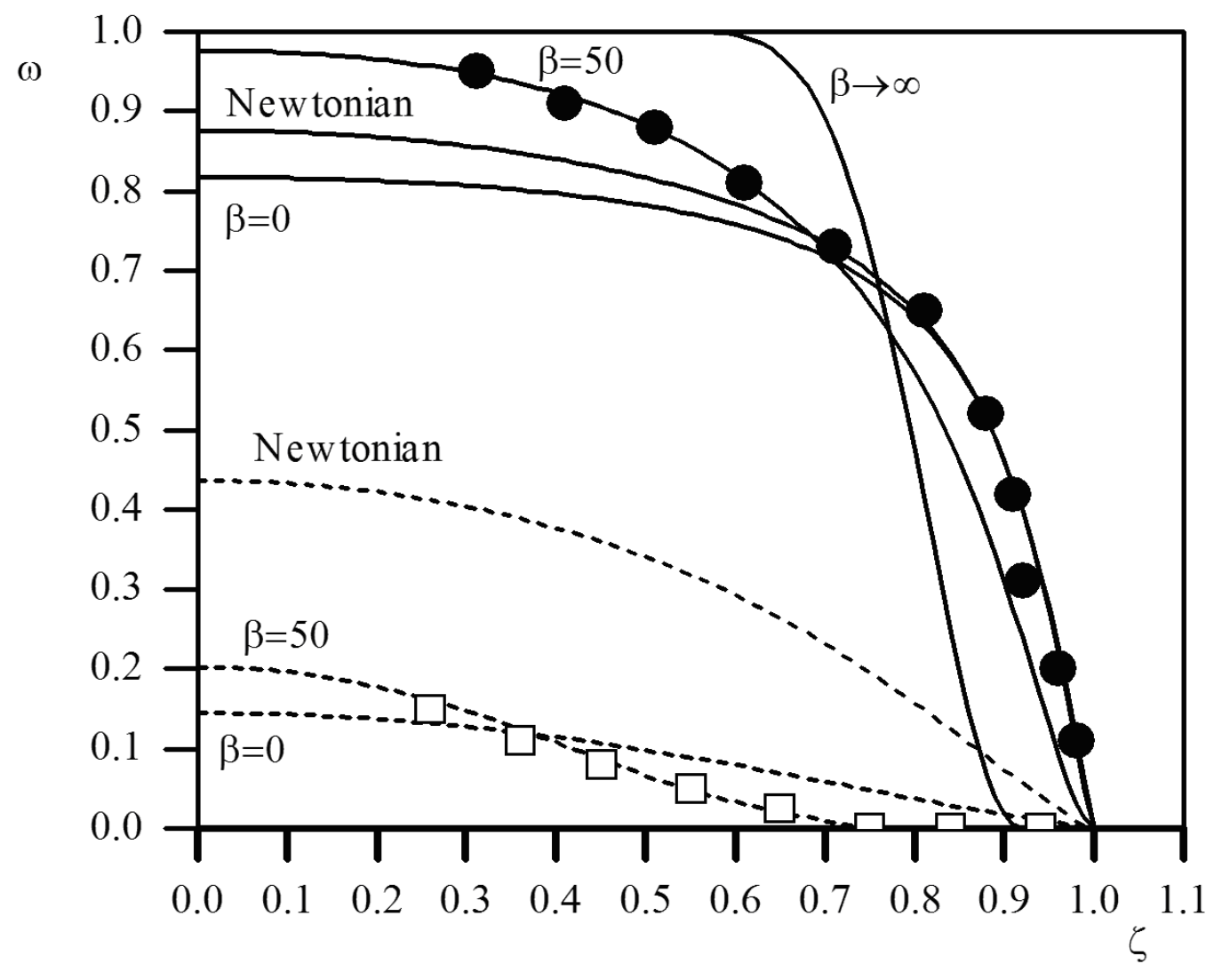

Figure 4.3. Comparison of various models to experimental data for rotating disk flow in a bed with $\alpha=0.88$. For symbols see Figure 4.2. The solid lines correspond to predictions for $\eta=0.792$; the dashed lines are for $\eta=0.510$. Predictions for $\beta \rightarrow \infty$ at $\eta=0.510$ coincide with the $\zeta$ axis. 


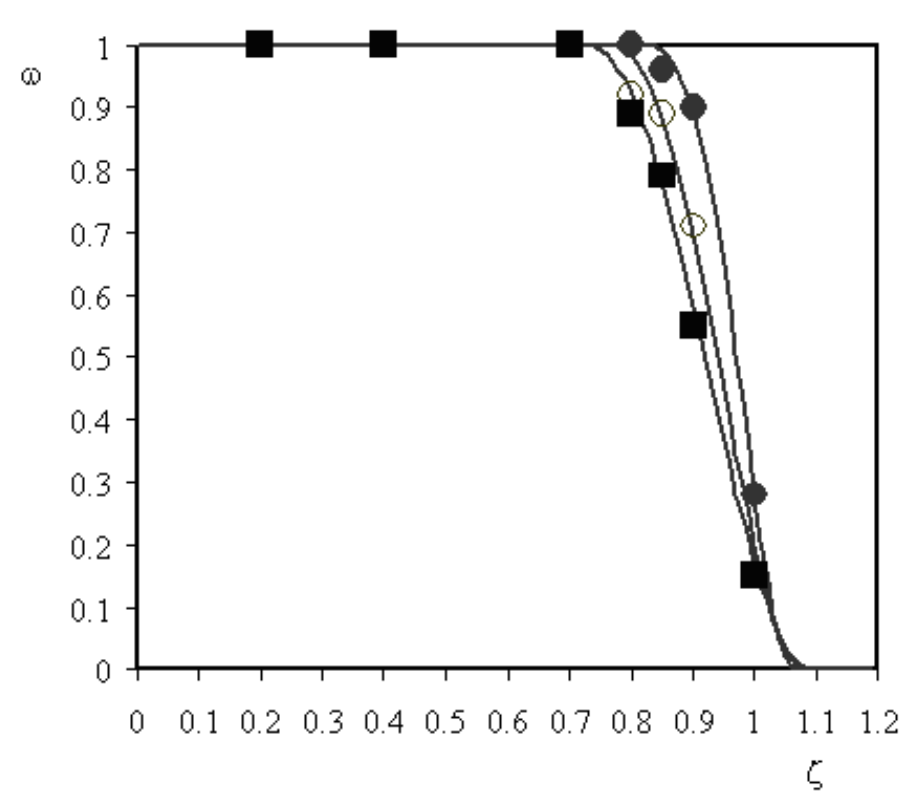

Figure 4.4. Experimental (symbols) [Cheng et al. (2006)] and predicted (solid lines, $\beta=50$ ) angular velocity profiles for rotating disk flow in a bed with $\alpha=0.4$. The symbols and accompanying lines correspond to: $\eta=0.316(\bullet), \eta=0.228(\circ), \eta=0.140$ (匹).

the point at which $\hat{\omega}_{0}$ starts to change with bed depth, as well as the rate at which the angular velocity drops. Even though the model tends to overpredict $\hat{\omega}_{0}$ for deep beds, the trend that this parameter follows is well represented.

The predictions presented in Figures 4.2-4.5 are based on calculations that treat the solids fraction, $v$, as uniform throughout the packing. In the formulation proposed herein, $v$ is a dependent variable coupled to both the pressure and the local shear rate by equation (4.7). For the current problem, assumption of uniform $v$ is not unreasonable, as a posteriori calculations of solids fraction variation with depth [by equation (4.7), using the value of a reported in reference 10] shows that $v$ was within $10 \%$ of $v_{\max }$.

Angular velocity predictions were also made using the Janssen pressure profile [equation (4.12)]. This adds an additional parameter to the model $(\lambda / R)$. However, inclusion of this additional parameter did not improve the prediction over results presented here within the feasible parameter space. This would indicate that the granular material in the experiments analyzed exhibited a nearly lithostatic pressure profile (not measured).

\subsection{Flow between Concentric Cylinders}

Mueth et al. (2000) presented detailed experimental measurements of velocity and solid fraction profiles in flow between concentric cylinders. These experiments consisted in the generation of a relatively narrow shear band by rotating the inner cylinder of a concentric cylinder (Couette) assembly filled with packings of monodisperse spheres that had a free surface open to the atmosphere. The shear band extended through a radial distance of about 10 particle diameters from the inner cylinder, leaving a sizable outer portion of the granular bed stationary.

For the application of the constitutive model, $v_{\theta}=v_{\theta}(r, z)$ is again considered the only nonzero component of the velocity. The linear momentum balance in the $\theta$ direction is equation (4.15). 


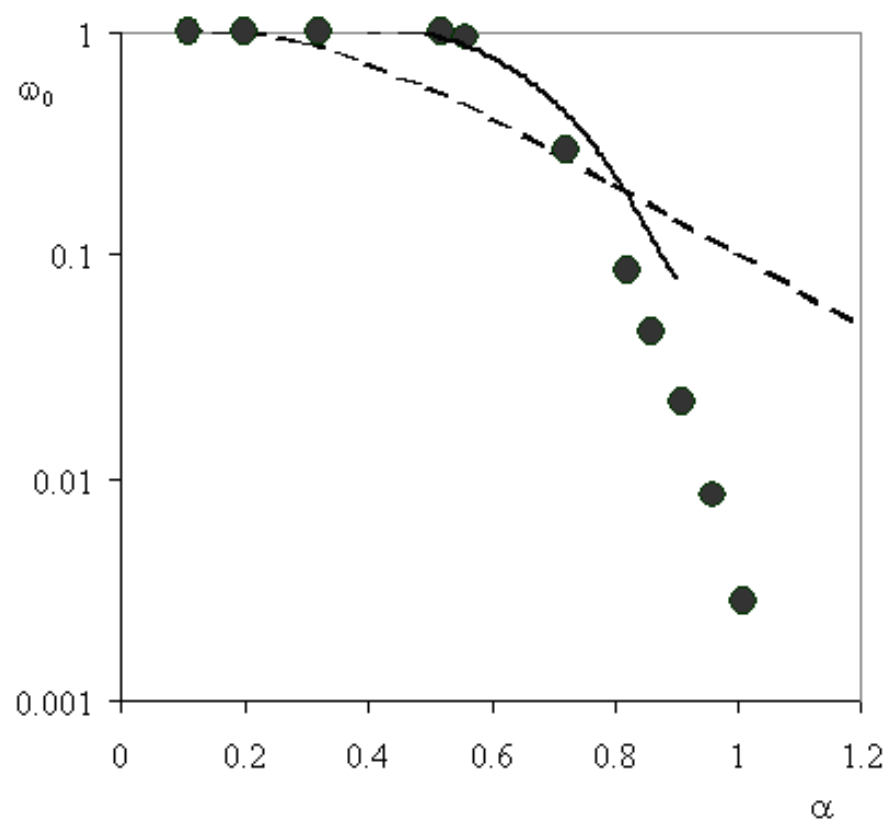

Figure 4.5. Angular velocity at the surface of the bed and cell axis for the experiments reported by Cheng et al. (2006): experimental data (•), model predictions (solid line) using $\beta=15$ (no adjustable parameters, see text). The dashed line represents the predictions for a Newtonian fluid.

In this case, however, since shear is applied uniformly in the $z$ direction by the rotating inner cylinder, it is reasonable to assume that radial variations of the velocity will be appreciably larger than vertical variations, which leads to consider that $\dot{\gamma}_{z \theta}<<\dot{\gamma}_{r \theta}$, an assumption that is verifiable once velocity profiles are calculated. Under these conditions, the second term of equation (4.15) can be neglected. Integration of the resulting equation, with the boundary condition

$$
\tau_{r \theta}=\tau_{v} \text {, at } r=R
$$

Here $R$ is the radius of the inner (rotating) cylinder and $\tau_{v}$ (unknown) is the shear stress applied at the rotating inner cylinder, yields

$$
\tau_{r \theta}=\tau_{v}\left(\frac{R}{r}\right)^{2}
$$

The shear stress will be represented by equations (4.10) and (4.11), with

$$
\dot{\gamma}=\left|\dot{\gamma}_{r \theta}\right|=\left|r \frac{\partial\left(v_{\theta} / r\right)}{\partial r}\right|
$$

Note that, in this case, since $\dot{\gamma}_{r \theta}<0, \dot{\gamma}=-\dot{\gamma}_{r \theta}$. 
The rest of the equations are expressed in dimensionless form, using the following definitions: $\hat{\omega}=v_{\theta} /(\Omega r), \zeta=r / R, \eta=z / R$, with $z=0$ being the free surface, and $T=\tau_{r \theta} /\left(\mu_{\text {min }}^{*} v_{\max } \rho_{p} g R\right)$, $T_{v}=\tau_{v} /\left(\mu_{\text {min }}^{*} v_{\max } \rho_{p} g R\right), \phi=v / \nu_{\max }$.

In the calculations presented here, variations of the solids fraction with position are considered. However, these changes are more significant in the radial direction than in the vertical direction, so that the pressure profile will be approximated by equation (4.13) instead of using the more rigorous integral form (equation 4.14).

Combining equations (4.10), (4.11), (4.13), and (4.33) yields, after manipulations

$$
\frac{\partial \hat{\omega}}{\partial \zeta}=\frac{\beta^{\prime}}{\zeta} \sqrt{\eta \phi}+\frac{T_{v} \beta^{\prime}}{\zeta^{3} \sqrt{\eta \phi}}
$$

where

$$
\beta^{\prime}=\frac{\mu_{\min }^{*} \sqrt{v_{\max } g R}}{b d \Omega}
$$

In addition, combining equations (4.7) and (4.13) yields, in dimensionless form,

$$
\frac{\partial \hat{\omega}}{\partial \zeta}=-\frac{\kappa \beta^{\prime}}{\zeta} \sqrt{\eta \phi}(1-\phi)
$$

where

$$
\kappa=\frac{b \mathrm{v}_{\text {max }}}{a \mu_{\text {min }}^{*}}
$$

Equations (4.35) and (4.37) can be combined to find $\phi$ and $\partial \hat{\omega} / \partial \zeta$ as a function of the dimensionless coordinates $\zeta$ and $\eta$, and the unknown parameter $T_{v}$. This parameter can be determined by recognizing that, since not all of the material is moving, there will be a yield surface at $\zeta=\zeta_{y}(\eta)$ where the shear rate and angular velocity will vanish. Using the condition

$$
\frac{\partial \hat{\omega}}{\partial \zeta}=0, \text { at } \zeta=\zeta_{y}
$$

in equation (4.35) leads to

$$
T_{v}=-\zeta_{y}^{2} \eta
$$


Note that $\mathrm{v}=1\left(\mathrm{v}=\mathrm{v}_{\max }\right)$ at $\zeta=\zeta_{y}$.

Substitution of equation (4.40) into equation (4.35), and elimination of $\phi$ with equation (4.37) yields a first order differential equation for $\hat{\omega}$ with the unknown $\zeta_{y}$. The velocity profile can be found by integrating the resulting equation with the boundary condition

$$
\hat{\omega}=1, \text { at } \zeta=1
$$

and the location of the yield surface, $y$, can be found by applying the condition

$$
\hat{\omega}=0, \text { at } \zeta=\zeta_{y}
$$

For the particular case in which the approximation $\phi \approx 1$ can be made in equation (4.35) [although not in equation (4.37), for obvious reasons], then equation (4.35) can be integrated directly [using equations (4.40)-(4.42)] to find $\hat{\omega}(\zeta, \eta)$, and then equation (4.37) yields explicitly $v(\eta, \zeta)$. This yields the approximate solution

$$
\hat{\omega}=1+\beta^{\prime} \sqrt{\eta}\left[\ln \zeta+\frac{1}{2}\left(\frac{\zeta_{y}}{\zeta}\right)-\frac{\zeta_{y}^{2}}{2}\right]
$$

with the yield surface obtained from the application of condition (4.42) to equation (4.43). Notice that, whenever this approximation holds, the dimensionless angular velocity profile can be fitted to experimental data by means of one adjustable parameter $\left(\beta^{\prime}\right)$; the solids fraction profile needs the additional parameter $\kappa$. The approximation (4.43) was not used in the solutions described herein. Instead, the system of equations was solved numerically.

Mueth et al. (2000) reported velocity and solid fraction profiles in shear bands formed over a cylinder of radius $R=51 \mathrm{~mm}$, rotating at an angular velocity around $\Omega=0.6 \mathrm{rad} / \mathrm{s}$. Packings of two different particles were used in the experiments: mustard seeds $(d=1.8 \mathrm{~mm})$, and poppy seeds $(d=0.8 \mathrm{~mm})$.

Figures 4.6 and 4.7 show fits of the model developed to experimental velocity and solids fraction profiles for two different particle beds made up of mustard and poppy seeds, respectively. The velocity profiles were fitted using $\beta^{\prime}$ as the only adjustable parameter, and the solids fraction profile fits used $\kappa$ as the only adjustable parameter. The values of $\beta^{\prime}$ and $\kappa$ were fitted independently for both experiments. However, since the only significant parametric differences between the two experiments are the particle size and the value of $\mathrm{v}_{\max }$, it would be expected that, provided that the dilatancy and friction equations (4.3) and (4.4) are universal correlations, both $\beta^{\prime}$ and $\kappa$ scale between the experiments according to equations (4.36) and (4.38). If the value of $\beta^{\prime}$ from Figure 4.6 (mustard seeds) is used to predict the corresponding value for the experiment in Figure 4.7 (poppy seeds), assuming that $b$ is the same, $\beta^{\prime}=96$ is obtained. This value is close to the fit provided $\left(\beta^{\prime}=85\right)$, which indicates that the friction law is similar for both packings. On the other hand, the value of $\kappa$ does not scale as expected: a prediction for the data in Figure 4.7 from the value obtained in Figure 4.6, assuming that the parameter $a$ remains constant, yields $\kappa=3.7$, 
which overestimates the fitted value of $\kappa=2.5$. A possible explanation for this finding would be a change in the dilatancy equation (4.4) for the poppy seeds. This packing is structurally different from the mustard seed packing because the poppy seeds used are not perfectly spherical. This is clearly seen in the much higher value of $v_{\text {max }}$ for this packing, which is indicative of a closer packing due to the irregular shape of the particles. A higher value of the parameter $a$ (i.e., a higher sensitivity of the dilatancy law to $I$ ) would explain the differences in $\kappa$ observed. However, there is not enough information at this point to confirm this claim.

\subsection{Conclusion}

The model described in this chapter is capable of capturing the flow fields of the rotating disk and concentric cylinders experiments using one adjustable parameter for the velocity field and an additional parameter to predict solids fraction profiles. For applications involving TRU sludge mobilization and transport, the continuum model developed in this chapter enables prediction of flow fields in a number of relevant configurations. Although validated against two specific geometries, the model should be applicable to any three dimensional flows meeting the requirements set forth herein. As such, velocity profiles existing around mechanical agitators can be predicted and used to judge the effectiveness of various mixing configurations. The model can also be applied to pipeline flow, including prediction of slurry flow profiles in pipe bends and joints. This indicates that the approach presented herein is a robust step towards establishing predictive methods for TRU waste sludge treatment, as well as a continuum constitutive representation of the rheology of cohesionless granular materials. However, the model is dependent on functional forms for scaling of frictional and dilatancy effects with inertial number obtained from two-dimensional granular media simulations. The overall adequacy of these relations has not been thoroughly tested, and would require a more extensive set of experimental data. In addition, it is important to point out that an analysis of the stress field in this type of flow is necessary to further explore the accuracy of the present postulate.
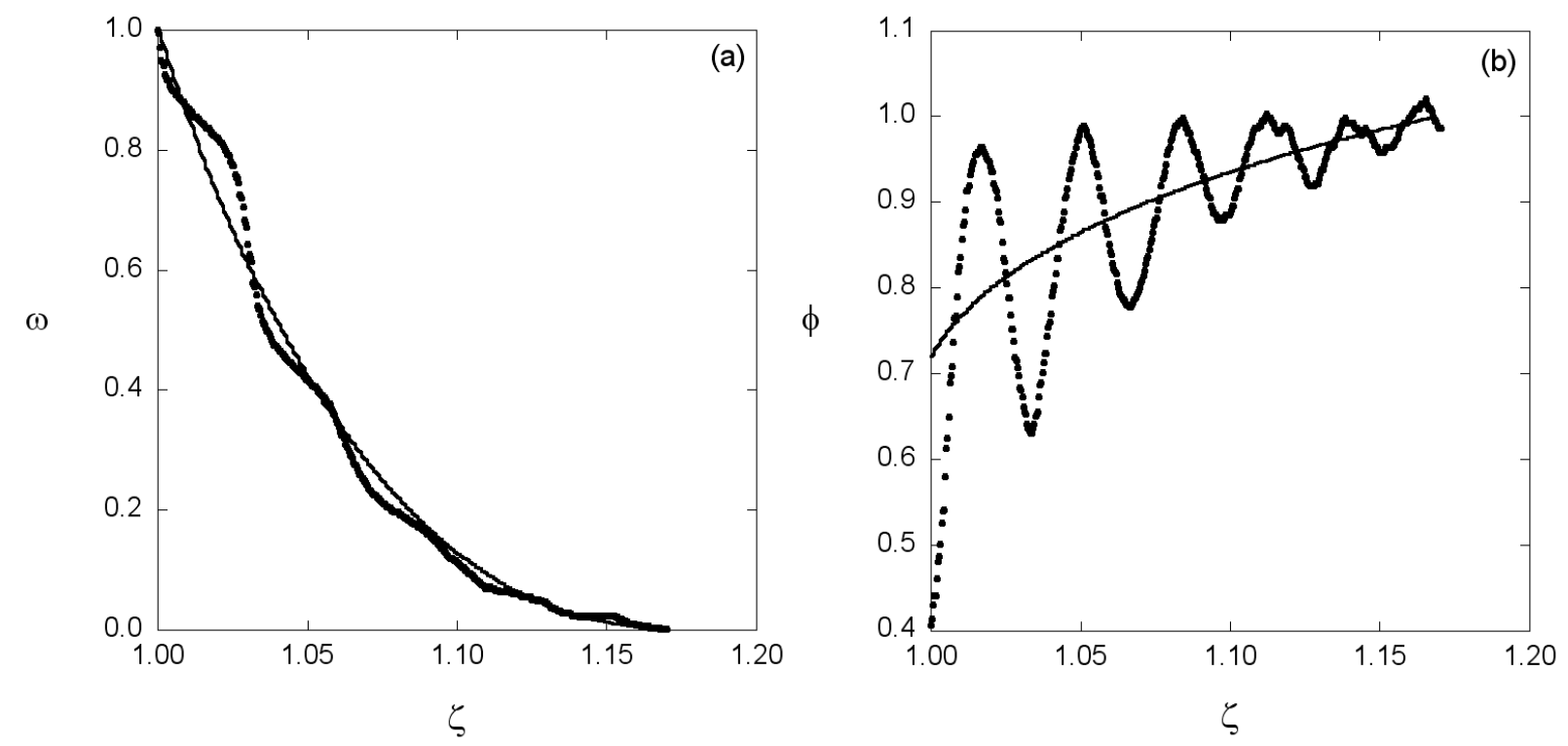

Figure 4.6. Experimental (symbols) [Mueth et al. (2000)] and fitted (solid lines) angular velocity (a) and solids fraction (b) profiles for flow between concentric cylinders of mustard seeds $\left(d=1.8 \mathrm{~mm}, v_{\max }=0.58\right)$ at $\eta=0.284$. The fits correspond to $\beta^{\prime}=40$ and $\kappa=3.2$. The oscillation in the experimental results is caused by the fact that measurements span just a few particles in the radial direction. 

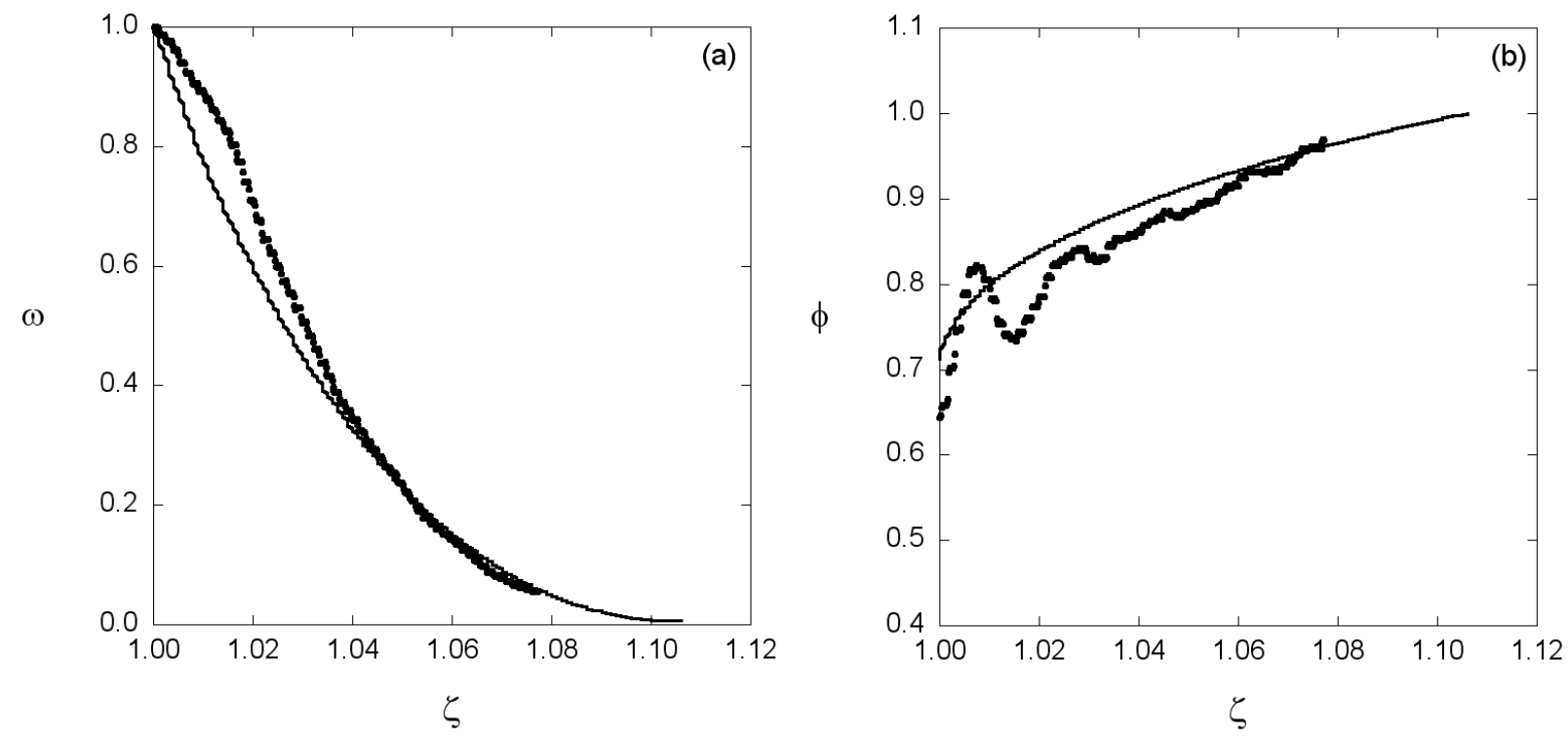

Figure 4.7. Experimental (symbols) [Mueth et al. (2000)] and fitted (solid lines) angular velocity (a) and solids fraction (b) profiles for flow between concentric cylinders of poppy seeds $\left(d=0.8 \mathrm{~mm}, v_{\max }=0.67\right)$ at $\eta=0.290$. The fits correspond to $\beta^{\prime}=85$ and $\kappa=2.5$.

The continuum model described in this chapter also provides a foundation by which the frictional and dilation properties of granular powders and slurries may be characterized. Specifically, the da Cruz parameters, $a$ and $b$, corresponding to the friction and dilation laws of the material under consideration, respectively, have been incorporated into a continuum model that can be applied to a large class of operating geometries. Determination of these parameters, especially the frictional parameter $b$, and application of the continuum model would facilitate scale-up of flow and stress dynamics from bench-scale to engineering-scale operations. Fluid properties are typically characterized using bench-scale rheometer operations such as Couette-flow devices and shear vane tools. However, as both this chapter and previous chapters show, characterization granular material using conventional rheometric techniques is not well developed. Chapter 3 described how to measure the failure strength for a granular solid using the vane technique. In the follow chapter, this technique is extended to characterizing the flow properties of fluids undergoing steady-shear. 



\subsection{Characterizing Cohessionless Powders with Vane Rheology}

In this chapter, the rheology and flow properties of a single coarse granular powder are characterized using a vane rheometer. The torque required to maintain constant rotation of a vane tool in a confined bed of glass beads (with a mean particle size of $203 \mu \mathrm{m}$ ) is measured as a function of vane immersion depth and rotational speed. The resulting torque profiles exhibit both quasi-static behavior at low rotational rates and fluid-like behavior at high rotational rates. Analyzing vane shaft and end effects allows the flow dynamics at the cylindrical and top and bottom disk surfaces of vane rotation to be determined. Disk surfaces show a uniform torque profile consistent with Coulombic friction over most of the rotational rates studied. In contrast, cylindrical surfaces show both frictional and collisional torque contributions, with significant dynamic torque increases at deep immersion depths and fast vane rotation. The continuum model presented in Chapter 4 of this report is used to model the system. Semi-quantitative prediction is achieved at rotational rates both below $0.5 \mathrm{rad} / \mathrm{s}$ and above $10 \mathrm{rad} / \mathrm{s}$. Separate approaches must be used at low and high rotational rates because the bed changes from quasi-static to partially fluidized conditions. Consistent with observations for static materials in Chapter 3, the bed appears to be governed by a Janssen type normal stress distribution such that pressure saturates for slow vane speeds at deep immersion depths. Indeed, the results indicate that normal stress profiles measured under static conditions provide a reasonable representation of the stress distributions for granular materials undergoing quasi-static flows. For fast vane rotation, the particles in the vicinity of the vane behave as if they were fully fluidized, and the normal stress distributions influencing granular rheology are primarily lithostatic. Prediction at rotational rates from $0.5 \mathrm{rad} / \mathrm{s}$ to $10 \mathrm{rad} / \mathrm{s}$ is complicated by changes in the granular microstructure and stress fields resulting from partial fluidization of the bed. Overall, it is possible to characterize the quasi-static and partially fluidized flow regimes with a vane rheometer. Knowledge of how the granular normal stress profile changes as the granular material is fluidized could enable prediction in the intermediate flow regime. It is hoped that this study provides a basis for characterizing more complicated systems, such as concentrated suspensions and slurries similar to and including Hanford TRU sludges.

\subsection{Background}

Characterizing the rheology of powders and granular slurries is complicated by the fact that the appropriate rheological description depends on the degree of fluidization and local shear of the material. Chapter 4 of this report addressed the continuum representation of three-dimensional granular flows in terms of mass and momentum conservation. This is still an area of extensive research (MiDi 2004, Depken et al. 2006, Sáez et al. 2006) as interest from its importance in engineering applications such as the design of hoppers and bins (Fayed and Otten 1997, Terzaghi 1943), mining and drilling (Barnes and Dzuy 2001), food processing (Steffe 1992), and waste remediation and disposal (Chang and Smith 1996). Chapter 3 discussed the important of the granular rheology in terms of Hanford TRU sludge mobilization, and many of the same concerns also extend to the mass transport of these sludges.

Again, for a mobilized cohesionless granular material, three basic flow regimes have been observed: quasi-static, intermediate, and kinetic. These regimes are described in Section 1.1 and further expanded throughout Chapter 4. The studies reference therein focus on measuring and predicting the spatial dependence of granular velocity fields and solids fraction profiles. Their main deficiency is that they lack general information on the stress-strain behavior of the granular material. With regard to design of sludge mixing and agitation systems, knowledge of the stress-strain behavior is crucial in determining power requirements for the mixing system. 
The goal of the work presented in this chapter is to characterize the stress-strain relationship of a single granular powder using a vane rheometer. This instrument was selected because of its simplicity of operation (see Section 3.1). For rotational vane rheometry, the shear surface is mainly composed of the granular material rotating with the vane blades. This provides a distinct advantage over comparable methods, such as Couette flow viscometry, which require an immobilized (i.e., "glued") layer of glass beads on the surface of the rheometer tool. The recent work by da Cruz et al. (da Cruz et al. 2005), Jop et al. (Jop et al. 2006), and Sáez et al. (Sáez et al. 2006) (described in the previous chapter) will be used to interpret and extend vane viscometry to granular solids.

The literature contains several studies (e.g., Benarie (Benarie 1961), Kuno and Kurihara (Kuno and Kurihara 1965), and Kuno and Senna (Kuno and Senna 1967)) concerning the characterization of granular matter using "vane" rotational viscometry. Benarie (Benarie 1961) examined the immersion depth and rotational rate dependence of the steady-state torque experienced by a rotating cylinder in contact with a cohesive granular solid. This study found that the torque required to maintain constant rotation showed a linear dependence on the amount of material above the rotor and did not depend on the rotational rate. The latter observation is consistent with the quasi-static dynamics. Intermediate flow conditions may not have been achieved because Benarie did not consider rotational rates above $1 \mathrm{rad} / \mathrm{s}$. Kuno and Kurihara (Kuno and Kurihara 1965) and Kuno and Senna (Kuno and Senna 1967) used a baffled cup and rotor system to characterize granular rheology, but, in contrast to Benarie's experiment, they rotated the cup instead of the rotor. They found an increase in the thickness of the sheared zone and an unexpected decrease in steady-state torque as the cup rotated more quickly. These unusual results may result from inertial forces generated by cup rotation. More recently, Poloski et al. (Poloski et al. 2006) employed the vane tool to characterize incipient motion of dry cohesionless glass beads. Similar to Benarie's findings for steady state flow, Poloski et al. determine that the torque required to initiate motion depends strongly on immersion depth and is relatively invariant with vane rotational rate.

There are a number of challenges to using the vane tool to measure granular rheology. Interpreting vane rheograms is difficult because of the dependence of stress-strain behavior on granular density and pressure as well as potential discontinuities in the velocity and solid-fraction profiles (Cheng and Farley 1965). Continuous contact networks formed between the particles transmit interparticle stress both laterally and vertically (Behringer et al. 1999). The intergranular pressure and the resulting frictional force between particles can vary significantly over the vertical depth of the bed. Frictional contact between the grains and the container supporting the material may also cause the local pressure to become dependent on the geometry of the container. Indeed, the effects of wall friction and immersion depth on the yield (failure) stress of granular materials has been demonstrated using the vane tool (as described in Chapter 3.1) as well as using other tools like the drawn-plate (Lee 1987).

The geometry of the region of granular material sheared by the vane is complex. A simplified view of the slip surface in Fig. 5.1 yields two basic flow problems: 1) the flow of granular material around the vane shaft and the cylinder of rotation formed by the vane blades on the $r=R$ surface and 2) the flow of granular material above and below the two disks of rotation formed on the $z=h$ and $z=h+H$ surfaces.

The problem of flow around a rotating cylinder has been studied experimentally by Mueth et al. (Mueth et al. 2000) and Mueth (Mueth 2003) using MRI. Under the conditions used in these works, the rotating cylinder only shears a small band of particles, typically 10 diameters wide. The adjacent material remains stationary. These studies only consider the microstructure and 
motion of the sheared region and do not measure either normal or shear stresses acting on the cylindrical surface, both of which are important from a rheological perspective.

Cheng et al. (Cheng et al. 2006) have studied flows of granular material in contact with a rotating disk using the same MRI technique employed in Refs. (Mueth et al. 2000, Mueth 2003). Cheng et al. found that the shear band shows no significant radial penetration beyond the radius of the inner rotating disk. Vertical shear assumes one of two configurations. If the bed is shallow, the sheared region penetrates the entire height of the material above the disk. If the bed is deep, the sheared region is contained in the interior of the granular solid such that the free surface is stationary. After Refs. (Mueth et al. 2000, Mueth 2003), Cheng et al. (Cheng et al. 2006) did not measure the stresses present in the system.

With regards to the vane technique, one important observation made in the MRI studies is that the radial shear band does not extend far beyond the cylinder of rotation. This suggests that the granular flow dynamics for the individual surfaces may not be coupled and, as such, could be considered separately. Despite the rich information provided by Mueth et al. and Cheng et al., a complete rheological treatment for vane rheometry is still missing where granular materials are concerned.

To the best of our knowledge, no studies have directly examined dynamic granular stress acting on a vane tool over a large range of rotational rates. This chapter describes how to interpret mathematically rheological flow curves for granular matter measured using a shear vane impeller tool. Torque profiles for cohesionless monodisperse glass beads were measured as a function of rotational rate, vane immersion depth, and blade height. Torque contributions for the top/bottom and radial shear surfaces were extrapolated from the data, and the results were analyzed in the

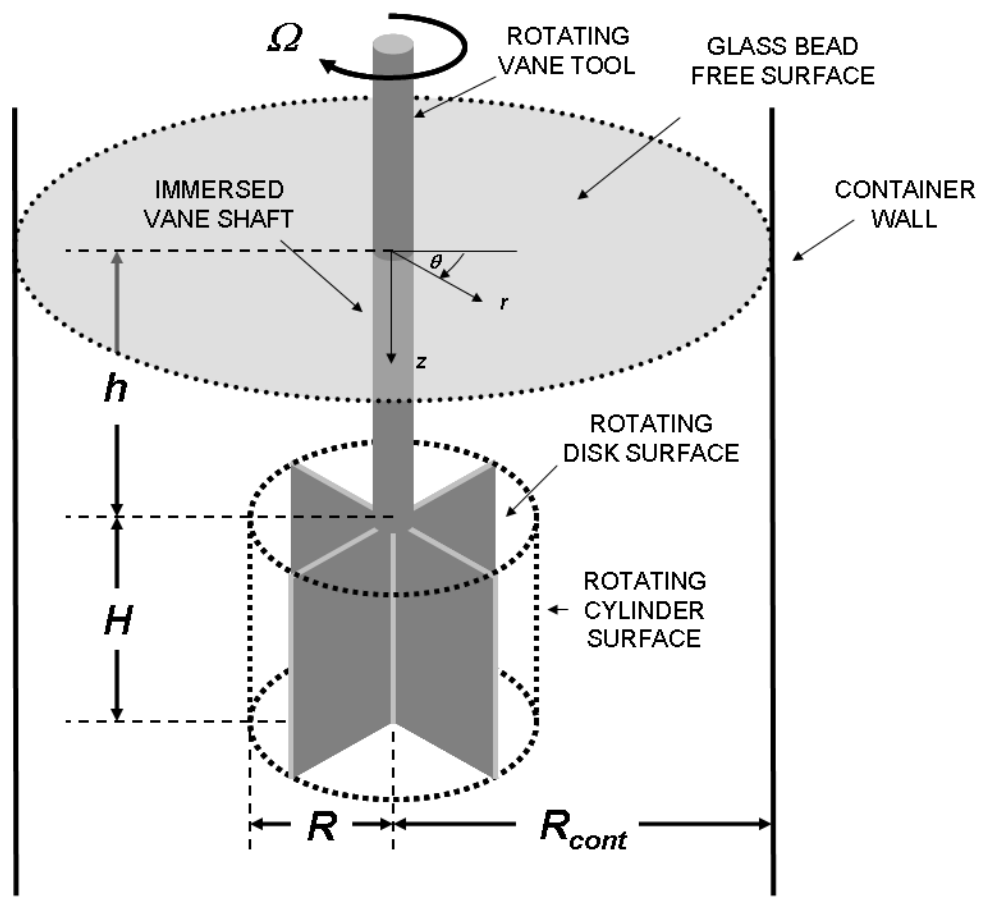

Figure 5.1. In a typical vane rheology test, a vane tool of radius $R$ and height $H$ is immersed to a depth $h$ in a sediment layer confined by a cylindrical wall with a radius of $R_{\text {cont }}$. The vane is rotated at a constant angular velocity $\Omega$, and the total torque $M_{\text {total }}$ required to maintain motion is recorded. Rotation of the vane sweeps out a cylinder, which is considered to be the shear surface. The total torque acting on the vane tool results from shear forces on the cylinder top, bottom, side, and shaft. 
context of a continuum model developed by Sáez et al. (Sáez et al. 2006) and described in Chapter 4.

\subsubsection{General Functionality of Vane Torque with Immersion Depth}

Vane rheograms for cohesionless powders are highly dependent on immersion depth. As seen in Chapter 3, a change in measured torque can be effected by changing the test depth or container geometry even in the absence of flow (Poloski et al. 2006). A set of relations with which to interpret and analyze this depth dependence can be derived by incorporating the influence of depth on $\mu^{*}$ into an apparent macroscopic friction coefficient. No explicit assumptions need to be made with regards to the rotational rate, and $\mu^{*}$ can still vary as a function of the local shear. Additionally, the effect of depth on the pressure is still included in the calculation of shear stress. A more general treatment, in which both the depth and shear dependence of $\mu^{*}$ are accounted for in rotating disk and cylinder geometries in granular media will be introduced in later sections of this chapter.

The vane tool is treated as an axisymmetric rigid body immersed into a dense granular bed (of known physical properties) contained in a cylinder of radius $R_{\text {cont }}$. The tool consists of a long shaft of radius $R_{S}$ connected to a blade impeller (Fig. 5.1). When rotated, the blades sweep out a cylinder of radius $R$ and height $H$. The immersed shaft length is $h$ and is measured relative to the top of the vane blades such that the total immersed length of the entire tool is $h+H$. The rotating vane is assumed to shear/mobilize only the material in the immediate vicinity of the vane such that the surrounding material remains stationary. A steady angular velocity of $\Omega$ is controlled by varying the applied torque $M_{\text {total }}$. The dynamic macroscopic friction coefficient is given by a representative value $\bar{\mu}^{*}$ over the entire length of immersion. Here, the superscript is used to differentiate $\bar{\mu}^{*}$ from $\mu^{*}$. Both friction coefficients allow for the same $\Omega$ functionality; however, $\bar{\mu}^{*}$ neglects the depth dependence of the $b I$ term in Eq. (4.3).

Using the equations developed in Chapter 4 to predict vane stresses requires knowledge of the static vertical and radial normal stress distributions, given by $P_{z z}$ and $P_{r r}$, respectively. If the stress network couples strongly with the confining walls (as is the case with deep beds under static or near static conditions), the vertical pressure $P_{z z}$ in a cylindrical container of radius $R_{\text {cont }}$ can be approximated by Janssen's equation, which again is,

$$
P_{z z}=v \rho_{p} g \lambda\left(1-e^{-z / \lambda}\right)
$$

As before in Chapters 3 and $4, \lambda$ is the Janssen screening length, given by

$$
\lambda=\frac{R_{\text {cont }}}{2 K \mu_{w}}
$$

Here, $\mu_{w}$ is the wall coefficient of friction, and $K$ is the Janssen coefficient. The latter defines the ratio of vertical-to-radial stresses such that radial normal stress, $P_{r r}$, is given by

$$
P_{r r}=K P_{z z}
$$


In regions where the stress network does not couple with the confining walls (i.e., for $z / \lambda<<1$ ), the lithostatic limit of pressure is valid,

$$
P_{z z}=v \rho_{p} g z
$$

The lithostatic limit will be used in the derivations below as its simplicity facilitates assessing the depth dependence of torque. Using either Eq. (5.1) or Eq. (5.4) assumes that the solids fraction does not experience significant variations in the $z$ direction. In addition, forces that can cause transient variation of normal stresses, such as particle jamming or crystallization forces, are neglected. Rotation of the vane is assumed to be sufficiently slow to allow radial inertia to be neglected as well. As such, the local pressure should be a function of depth alone.

The total torque acting on the vane will be split into torque contributions associated with the cylinder of rotation formed by the vane blades (i.e., side contributions), the disks of rotation formed by the upper and lower parts of the vane blades (i.e., top and bottom contributions), and the vane shaft (shaft contributions). In typical vane rheology measurements, the cylindrical surface described by the tip of the rotating vane is treated as the shearing surface. For the current analysis, the portion of the shaft connecting the vane to the rheometer in contact with the granular solid is also considered. Based on the apparent friction coefficient, $\bar{\mu}^{*}$, the shear stresses acting on vertical and radial elements would be [from Eq. (4.2)]

$$
\begin{gathered}
\tau_{z \theta}=\bar{\mu}^{*} v \rho_{p} g z \\
\tau_{r \theta}=\bar{\mu}^{*} K v \rho_{p} g z
\end{gathered}
$$

From these two equations, expressions for torque contributions from the top and bottom disks ( $M_{\text {top }}$ and $M_{\text {bot }}$, respectively) as well as contributions from the shaft and cylinder surface of rotation $\left(M_{\text {shaft }}\right.$ and $\left.M_{\text {side }}\right)$ may be determined. The contributions from the vane ends for a vane tool immersed to a depth $h$ are

$$
\begin{aligned}
& \left.M_{t o p}\right|_{h}=\frac{2 \pi}{3}\left(R^{3}-R_{s}^{3}\right) \bar{\mu}^{*} v \rho_{p} g h \\
& \left.M_{b o t}\right|_{h}=\frac{2 \pi}{3} R^{3} \bar{\mu}^{*} v \rho_{p} g(h+H)
\end{aligned}
$$

The appearance of $R_{s}$ in the expression for top torque accounts for the loss of disk area due to the vane shaft. In both cases, torque scales linearly with immersion depth. Contributions from the 


$$
\begin{gathered}
\left.M_{\text {shaft }}\right|_{h}=\pi R_{s}^{2} \bar{\mu}^{*} K v \rho_{p} g h^{2} \\
\left.M_{\text {side }}\right|_{h}=\pi R^{2} \bar{\mu}^{*} K v \rho_{p} g\left(H^{2}+2 H h\right)
\end{gathered}
$$

From Eq. (5.9), it can be seen that the shaft torque scales quadratically with immersion depth and has a zero intercept whereas side torque scales linearly with $h$ and is offset from the origin by a value proportional to the square of the vane height, $H$. The functional dependence of Eq. (5.10) on $H$ will be important for experimental determination of the vane end effects.

The treatment above employs an apparent friction coefficient, $\bar{\mu}^{*}$, that neglects the depth dependence of the inertial number [Eq. (4.1)]. While this is done to gain an overall picture of the depth dependence of dynamic torque, there are circumstances under which Eqs. (5.7 to 5.10) are valid. Specifically, the contribution of $b I$ to $\mu^{*}$ can be neglected without introducing uncertainty into $\mu^{*}$ at sufficiently low rotational rates (i.e., when $I$ approaches zero, and Coulombic granular flow occurs). Here, the bulk friction coefficient is nearly $\mu_{\min }^{*}$ at all depths as well as all vane rotational rates over the quasi-static flow regime. The substitution $\bar{\mu}^{*}=\mu_{\min }^{*}$ allows Eqs. (5.7 to 5.10) to be applied to quasi-static flows without ambiguity as to their validity.

\subsubsection{Determination of Vane Shaft and End Effects}

The typical approach to vane rheology is to combine all of the torque contribution equations into a single relationship (Steffe 1992), which is then compared against the total measured torque acting on the tool. Indeed, this is the approach taken in Chapter 3 of this report. To better understand how the vane interacts with the granular material at each surface, the approach herein will be to determine torque contributions from each of the surfaces of interest, namely the shaft, the disks, and the cylinder. It should be noted that the shaft contribution is the only total torque component that can be measured directly. This is accomplished by using a vane tool without blades. Vane end effects and the torque on the vane side must be determined by analyzing how vane geometry and immersion depth affects the total vane torque.

In terms of the individual contributions from the shaft, disk, and cylinder surfaces, the total vane torque at an immersion depth of $h$, is

$$
\left.M_{\text {total }}\right|_{h}=\left.M_{\text {side }}\right|_{h}+\left.M_{\text {top }}\right|_{h}+\left.M_{\text {bot }}\right|_{h}+\left.M_{\text {shaft }}\right|_{h}
$$

As stated before, $M_{\text {total }}(h)$ and $M_{\text {shaft }}(h)$ can be experimentally measured as a function of immersion depth. This leaves the top, bottom, and side torques as unknowns.

The dependence of the vane end contributions (i.e., top and bottom torques) on $h$ can be determined experimentally by considering how the total vane torque varies with vane height $H$. As seen in Eq. (5.10), the cylinder torque contribution vanishes as the vane height $H$ approaches zero, and the equations for top and bottom vane contributions converge in the limit of small $H$. 
In terms of Eq. (5.11), the torque acting on a vane immersed to a depth of $h$ whose height, $H$, approaches zero should be:

$$
\left.\left[\left.M_{\text {total }}\right|_{h}\right]_{H \rightarrow 0} \approx M_{\text {top }}\right|_{h}+\left[\left.M_{\text {bot }}\right|_{h}\right]_{H \rightarrow 0}+\left.M_{\text {shaft }}\right|_{h}
$$

$M_{\text {top }}(h)$ and $\left[M_{\text {bot }}(h)\right]_{H \rightarrow 0}$ would be equal in an isotropic fluid; however, because there is a vertical stress gradient throughout the granular material, these two contributions may differ based on differences in the vertical translation of shear in the $-z$ direction for the top disk and in the $+z$ for the bottom disk. This analysis assumes that both are similar enough such that a single averaged value, defined as $M_{\text {end }}(h)$, can represent disk surface contributions at an immersion depth, $h$. Then,

$$
\left.2 M_{\text {end }}\right|_{h}=\left.M_{\text {top }}\right|_{h}+\left[\left.M_{\text {bot }}\right|_{h}\right]_{H \rightarrow 0}
$$

So that

$$
\left.M_{\text {end }}\right|_{h}=\frac{\left[\left.M_{\text {total }}\right|_{h}\right]_{H \rightarrow 0}-\left.M_{\text {shaft }}\right|_{h}}{2}
$$

The functional form of the end effects may be determined by averaging Eqs. (5.7) and (5.8) for $H=0$ :

$$
\left.M_{\text {end }}\right|_{h}=\frac{\pi}{3}\left(2 R^{3}-R_{s}^{3}\right) \bar{\mu}^{*} v \rho_{p} g h
$$

Similar to the top surface contribution, the averaged vane end effects should be linear in $h$ and have a zero intercept.

The averaged top and bottom vane contributions allow the top and bottom disk contribution to be determined for a vane of height, $H$, immersed to a depth, $h$, through:

$$
\begin{gathered}
\left.M_{\text {top }}\right|_{h}=\left.M_{\text {end }}\right|_{h} \\
\left.M_{\text {bot }}\right|_{h}=\left.M_{e n d}\right|_{h+H}
\end{gathered}
$$

From these equations, the torque from the cylinder may be determined using

$$
\left.M_{\text {side }}\right|_{h}=\left.M_{\text {total }}\right|_{h}-\left.M_{\text {end }}\right|_{h}-\left.M_{\text {end }}\right|_{h+H}-\left.M_{\text {shaft }}\right|_{h}
$$


Due to the anisotropy of stresses in granular media, determining cylinder and end (disk) effects is more involved than for typical rotational viscometry (cf. Ref. (Steffe 1992)). Instead of a single value for the tool end effects, the change in granular pressure necessitates knowledge of depth dependence of the end effects. The result is that vane characterization of granular matter requires rheograms for a range of immersion depths and tool heights.

\subsection{Methods and Materials}

A vane rheometer was used to characterize the flow behavior of a cohesionless $203 \mu \mathrm{m}$ glass bead powder. The results and observations derived from this study are then used in the prediction of vane rheograms for a dense slurry of $203 \mu \mathrm{m}$ glass beads in deionized water. Experimental details for measuring the vane rheographs and determining the mechanical/frictional properties of the powder are given in the following paragraphs.

\subsubsection{Measurement of Dry Glass Bead Flow Curves}

The granular solid used for this study was a Spheriglass ${ }^{\circledR} 1922$ Type-A glass bead powder obtained from Potters Industries, Inc. (Valley Forge, PA 19482-0840). Spheriglass ${ }^{\circledR}$ Type-A beads are a nonporous soda-lime glass with a particle density of $2.5 \mathrm{~g} / \mathrm{cm}^{3}$, a dry bulk density of approximately $1.45 \mathrm{~g} / \mathrm{cm}^{3}$, and a mean particle size of $203 \mu \mathrm{m}$.

Flow curves for the dry powder were measured using a Haake M5 viscometer fitted with fourbladed shear vanes of varying height. Vane dimensions satisfy the vane criteria outlined in Steffe (Steffe 1992). The M5 system was configured to monitor the torque required to maintain a set rotational rate. Approximately $1.8 \mathrm{~L}$ of glass beads were poured into a $2 \mathrm{~L}$ Pyrex ${ }^{\circledR}$ beaker (radius of $70 \mathrm{~mm}$ ). The filled beaker was placed on an adjustable laboratory stand positioned below the viscometer, and the stand was slowly raised until the vane was immersed to a desired depth. An illustration of the experimental setup is given in Fig. 5.2.

Steady-state torque measurements were taken at rotational rates spanning $0.03 \mathrm{rad} / \mathrm{s}$ to $50 \mathrm{rad} / \mathrm{s}$. At least three replicate measurements were taken at each rotational rate. To verify that all experiments started with a similar granular packing structure, the glass beads were emptied into a separate container and then poured back into the original container before each test. Vane immersion depths typically fell between $10 \mathrm{~mm}$ and $90 \mathrm{~mm}$. Tests were carried out using stainless steel vane tools with impeller diameters of $16 \mathrm{~mm}$ and heights ranging from $2 \mathrm{~mm}$ to $32 \mathrm{~mm}$. All tools employed a shaft $3 \mathrm{~mm}$ in radius and $10 \mathrm{~mm}$ in length. The one exception was the $32 \mathrm{~mm}$ diameter tool, which had a shorter shaft $6 \mathrm{~mm}$ in length. Shaft contributions were determined by repeating the rheometric studies outlined above with a vane tool without blades, hereafter called the shaft tool.

Vane end effects are extrapolated from the measurements of total torque for different vane heights. $\left[M_{\text {total }}(h)\right]_{H \rightarrow 0}$ is determined by fitting $M_{\text {total }}$ versus $H^{2}$ over vane heights of $2 \mathrm{~mm}$ to $32 \mathrm{~mm}$ for $h<6 \mathrm{~mm}$ and $2 \mathrm{~mm}$ to $16 \mathrm{~mm}$ for $h \geq 6 \mathrm{~mm}$. Consistent with Eq. (5.10), all fits were constrained such that both the curvature and slope were positive for $H>0$.

Vane rheograms were also measured for a slurry of the Spheriglass ${ }^{\circledR}$ glass beads in water. As in the studies presented in Chapter 3, the glass/water slurries were prepared by oversaturating the powder with deionized water to eliminate the possibility of interparticle capillary forces. Slurry systems were fully mixed prior to testing. The shear vane was inserted at a specific depth approximately 2 minutes after mixing to minimize settling and packing. Vane rheograms were measured using the same methodology as the dry glass bead powder experiments. Once 


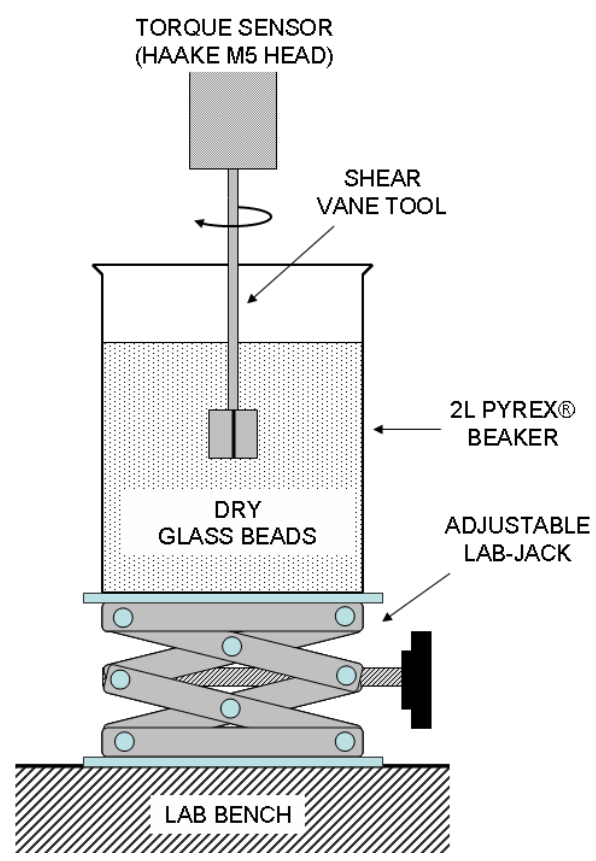

Figure 5.2. An illustration of the experimental setup used for the vane rheology tests. A $2 \mathrm{~L}$ Pyrex ${ }^{\circledR}$ beaker filled with a $203 \mu$ m powder is placed on an adjustable lab-jack directly beneath the measuring system. The beaker is raised until the vane is immersed to the desired test depth. Steady-state stepped-flow curves were operated over $0.03 \mathrm{rad} / \mathrm{s}$ to $50 \mathrm{rad} / \mathrm{s}$ to determine the glass bead torque-rotational rate profiles.

the shear strength measurement was completed, the vane was removed and the test sediment resuspended using a large spoon vertical bottom-to-top lifting motion (i.e., by ladling the slurry). Unlike with the dry powder, torque measurements for the slurry system was not broken down into contributions from the shaft and cylinder and disk surfaces of rotation. Instead, the total vane rheograms will be modeled directly based on the results of the dry glass bead system.

\subsubsection{Measurement of Static Granular Pressure Distribution}

The static granular pressure distribution is important for predicting granular stresses. The radial normal stress profile, $P_{r r}$ for the Spheriglass ${ }^{\circledR}$ powder was measured directly using a LCL-113G Full Bridge Thin Beam Load Cell from OMEGA Engineering, Inc. (Stamford, Connecticut 06907-0047) mounted on a stainless steel bar. The force sensor was wrapped in a LLDPE plastic sheath (with a thickness of 2 mil) to prevent intrusion by glass beads. Sensor force/pressure readings were calibrated against the hydrostatic pressure of water.

The experimental setup used for these measurements is similar to that for dynamic vane torque measurements shown in Fig. 5.2. Here the vane was replaced by the force sensor. Measurements were conducted by first positioning the force sensor at the center of an empty Pyrex ${ }^{\circledR}$ beaker of volume $2 \mathrm{~L}$. The sensor face was aligned with the beaker axis of symmetry so that radial forces/stresses were measured. Approximately $15 \mathrm{~mm}$ of clearance was left between the bottom of the force sensor and the floor of the beaker. The beaker was filled with glass beads until the sensor was immersed to a desired depth. The force acting on the thin beam load cell was recorded and converted to pressure using the hydrostatic calibration curve. The force sensor was removed, the glass beads were poured into another container, and the steps outlined above were repeated in subsequent tests. Granular forces were measured at immersion depths spanning $10 \mathrm{~mm}$ to $90 \mathrm{~mm}$, with at least five replicate measurements taken at each point. 


\subsection{Results and Discussion}

The results of the dry glass bead powder experiments outlined in the preceding sections can be separated into three categories: 1) pressure distribution and frictional properties of the $203-\mu \mathrm{m}$ powder, 2) total vane torque and torque contributions from the disk and cylinder vane surfaces, and 3 ) modeling of the total torque. These are discussed in detail in the following sections. The chapter is concluded by applying the results of the dry glass bead experiments in the prediction of vane rheograms for the glass/water slurry system.

\subsubsection{Static Stress and Frictional Properties for the Glass Bead Powder}

Figure 5.3a shows the radial normal stress profile measured by the thin-beam load cell immersed in a static bed of particles at various depths. Each measurement has an associated uncertainty (as determined by its standard deviation) ranging from $15 \%$ at deep immersions to $30 \%$ at the shallow immersions. Significant deviations are expected for granular solids because of the anisotropic nature of the stress field. The error at the largest immersion depth is similar to the 10 to $15 \%$ error observed in analogous experiments where vane torque is measured [cf. Poloski et. al. (Poloski et al. 2006)]. The high uncertainty at shallow depths likely derives from a combination of error from packing nonuniformity and from the limit of pressure sensor sensitivity, which is estimated to be around $\pm 10 \mathrm{~Pa}$.

The profile's downward curvature suggests stress saturation typical of granular materials contained by a cylindrical wall. Quantitative descriptors for the profile are obtained by determining values of $K$ and $\mu_{w}$ that yield a best fit of the data to Eq. (5.1). Regressed values of $K$ and $\mu_{w}$ are 0.6 and 0.7 , respectively. The radial normal stress profile calculated using these values (shown by the solid line in Fig. 5.3a) adequately reproduces the magnitude and curvature of the experimental data.

Comparison against the stress profile inferred from torque measurements in previous works by Poloski et al. (Poloski et al. 2006) for the same granular system is also given in Fig. 5.3a. The result of Poloski et al., given by the dashed line, is calculated using $K=0.39$ and $\mu_{w}=0.95$ and falls below the lower bounds of the error associated with the stress measurements. The difference is a result of the treatment of stress anisotropy. Poloski et al. regress values of $K$ and $\mu_{w}$ from the sum of torques acting on vertical and radial surfaces. The exact degree of vertical to radial force transmission (embodied by $K$ ) is made ambiguous by the need to also regress a macroscopic coefficient of static friction, $C_{f}$ (defined as the ratio of shear stress to pressure under static conditions). Indeed, nearly the same fit of the torque versus depth profile can be achieved using either the Janssen parameters determined directly or those inferred by Poloski et al. by simply adjusting the value of $C_{f}$, as shown in Fig. 5.3b.

Figure 5.3 shows the stress profile for the granular medium in a static state. For the analysis of vane rheology that follows, this profile will be treated as a best estimate of the radial (and inferred vertical) forces acting on the vane tool. As discussed in Chapter 9 of Fayed and Otten (Fayed and Otten 1997) and more recently by Behringer et al. (Bertho et al. 2003), the pressure distributions in cylinders containing flowing granular solids can still be described with a Janssen profile, although the associated $K$ and $\mu_{w}$ take values bound within static and kinetic friction limits. For glass-against-glass frictional interactions, these are $0.25<K<0.60$ and $0.35<\mu_{w}<0.95$. Experimental observations found that only the first $10 \mathrm{~mm}$ of material near the vane is sheared, with the adjacent material static. As such, it will be assumed that the normal stress profile effecting granular rheology in the vicinity of the vane can be reasonably approximated by the solid curve regressed in Fig. 5.3. Change in the state of stress is expected 

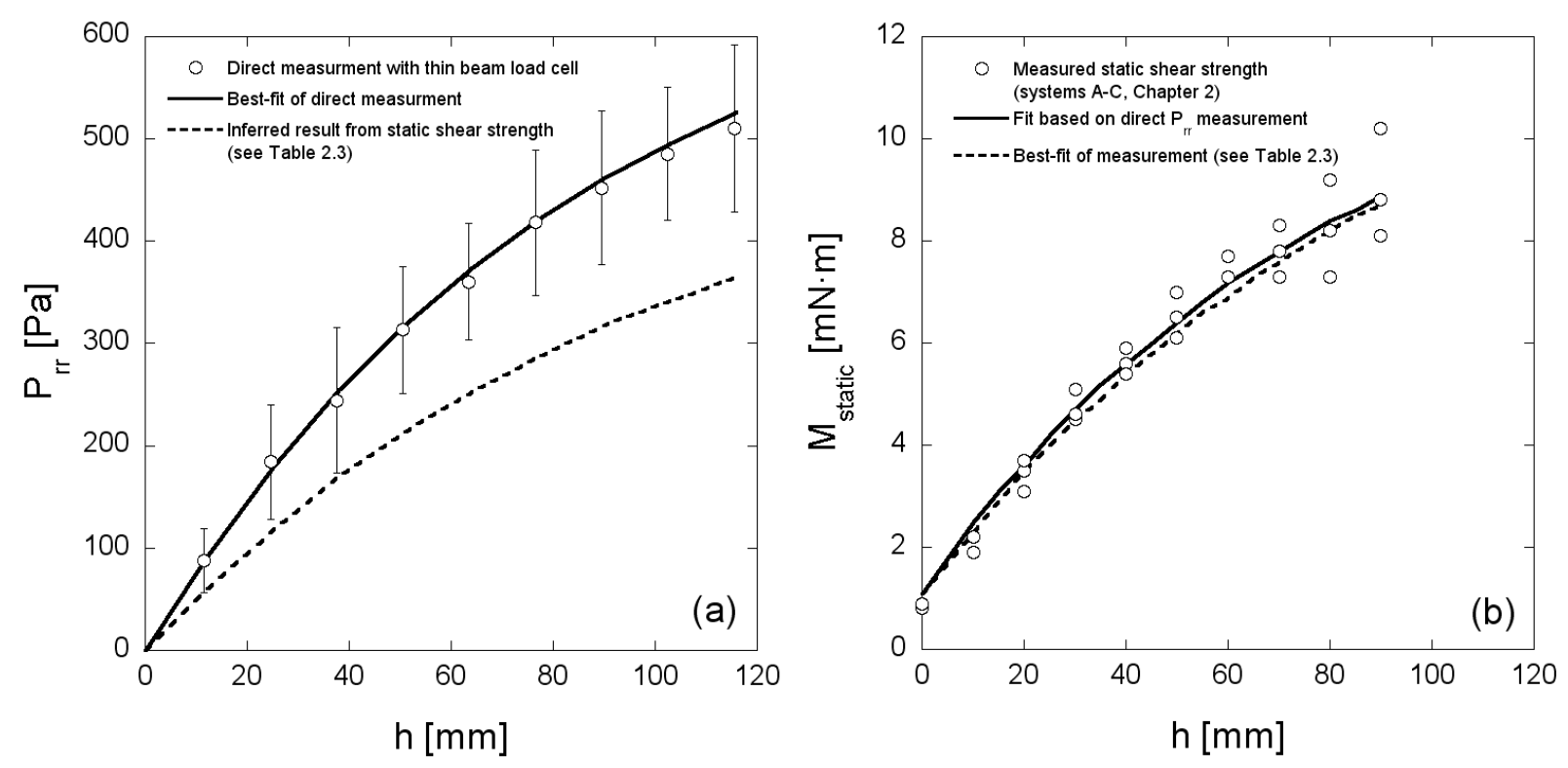

Figure 5.3. Measured (a) radial normal stress and (b) static shear strength (torque) as a function of immersion depth for a $203-\mu \mathrm{m}$ glass-bead powder. Curves in part (a) correspond to pressure profiles calculated using Janssen's law [see Eq. (5.1)] with $K$ and $\mu_{w}$ regressed from direct stress measurement (solid line: $K=0.6, \mu_{w}=0.7$ ) and those inferred from analysis of torque profiles for Systems A-C in Chapter 1 (dashed line: $K=0.39, \mu_{w}=0.95$ ). Curves in part (b) are torque profiles calculated from direct stress measurements (solid line: $C_{f}=1.8, K=0.6, \mu_{w}=0.7$ ) and from the results presented in Chapter 1 (dashed line: $C_{f}=2.2, K=0.39, \mu_{w}=0.95$ ).

because of material densification from vane vibration and shifts in the granular stress chains under dynamic conditions. It would be preferable to determine the dynamic normal stress at the vane surface; however, such measurements are not feasible with the present technique as the size of the pressure sensor (which is comparable to the vane) would alter the flow and stress fields.

The assumption of static $K$ and $\mu_{w}$ defines the pressure profile; however, the limit of kinetic friction at zero flow $\left(\mu_{\text {min }}^{*}\right)$ must also be known to calculate and model torque. In the limit of Coulombic flow (i.e., $I \rightarrow 0$ ), Eq. (4.2) reduces to $S=\mu_{\min }^{*} P$. This stress equation is equivalent to that developed in Poloski et al. (Poloski et al. 2006) for a system without particle cohesion, with $\mu_{\text {min }}^{*}$ being substituted for Poloski's $C_{f}$ parameter. Although their analysis is applied only to incipient motion of powders, it should be equally applicable to dynamic systems so long as its use is confined to regimes where the measured torque is independent of vane rotational rate. For the dynamic systems under consideration here, the total torque acting on a vane tool interacting with a material that obeys Janssen's equation would be given by (Poloski et al. 2006)

$$
M_{\text {total }}=\mu_{\min }^{*} X
$$

with 


$$
\begin{aligned}
\frac{X}{2 \pi \nu \rho_{p} \lambda}=\quad & K\left(H+\lambda e^{-\frac{h+H}{\lambda}}-\lambda e^{-\frac{h}{\lambda}}\right) R^{2}+ \\
& K\left(H+\lambda e^{-\frac{h}{\lambda}}-\lambda\right) R_{s}^{2}+ \\
& \frac{1}{3}\left(2-e^{-\frac{h+H}{\lambda}}-e^{-\frac{h}{\lambda}}\right) R^{3}- \\
& \frac{1}{3}\left(1-e^{-\frac{h}{\lambda}}\right) R_{s}^{3}
\end{aligned}
$$

Again, $M_{\text {total }}$ represents the total torque, including contributions from the disk and cylindrical surfaces and the vane shaft. The form of $X$ is selected so that plots of torque as a function of the calculated $X$ parameter yield a straight line with zero intercept. This allows trivial regression for the fitting of $\mu_{\text {min }}^{*}$ from torque measurements at low rotational rates.

Figure 5.4 shows application of Eq. (5.19) to equilibrium torques derived from experiments where the shear vane was rotated at $0.03 \mathrm{rad} / \mathrm{s}$. The independence of torque on rotational rate was confirmed by examining points above and below $0.03 \mathrm{rad} / \mathrm{s}$ (see Table 5.1). Vane immersion depths ranging from $10 \mathrm{~mm}$ to $90 \mathrm{~mm}$ were tested using vane tools with differing heights $(H=2-32 \mathrm{~mm})$. Regression of the entire data set yields a best-fit $\mu_{\min }^{*}$ of 0.8 . From the plot, it is evident that certain sets of data deviate from the best-fit. Typically, the shorter vane tools result in lower torques for the same $X$ value. The downward curvature observed in some of the data suggests a change in the stress distribution, which affects rheology under dynamic conditions. This would not be captured in the calculation of $X$ because of static $K$ and $\mu_{w}$. The data's lower and upper limits of variation are bound by $\mu_{\text {min }}^{*}$ of 0.5 and 1.0 , respectively. A $\mu_{\min }^{*}$ of 0.8 will be used in predictive calculations in this chapter.

\subsubsection{Vane Tool Torque Profiles}

Figure 5.5 shows the total measured torque acting on a vane tool with $R=8 \mathrm{~mm}$ and $H=16 \mathrm{~mm}$ over rotational rates spanning $0.03 \mathrm{rad} / \mathrm{s}$ to $50 \mathrm{rad} / \mathrm{s}$. The different profiles correspond to different vane immersion depths. The rotational rate is plotted using a logarithmic scale to better illustrate the changes in observed flow behavior. It is clear that the stresses acting on the vane are a function of both rotational rate and vane immersion depth. Torque always increases with increasing immersion depth. The rotational rate dependence of the torque profiles is complex, but can be classified into three regions:

Table 5.1. Low rotational rate torque measured using an $8 \times 16(R \times H, m m \times m m)$ shear vane at an immersion depth of $20 \mathrm{~mm}$. These measurements verify that data in Fig. 5.4 correspond to the quasi-static flow region.

\begin{tabular}{ll}
\hline$\Omega(\mathrm{rad} / \mathrm{s})$ & Equilibrium Torque $(\mathrm{mN} \cdot \mathrm{m})$ \\
\hline 0.017 & $1.9 \pm 0.1$ \\
0.035 & $2.1 \pm 0.1$ \\
0.070 & $2.0 \pm 0.1$ \\
0.140 & $2.1 \pm 0.1$ \\
0.279 & $2.0 \pm 0.1$ \\
\hline
\end{tabular}




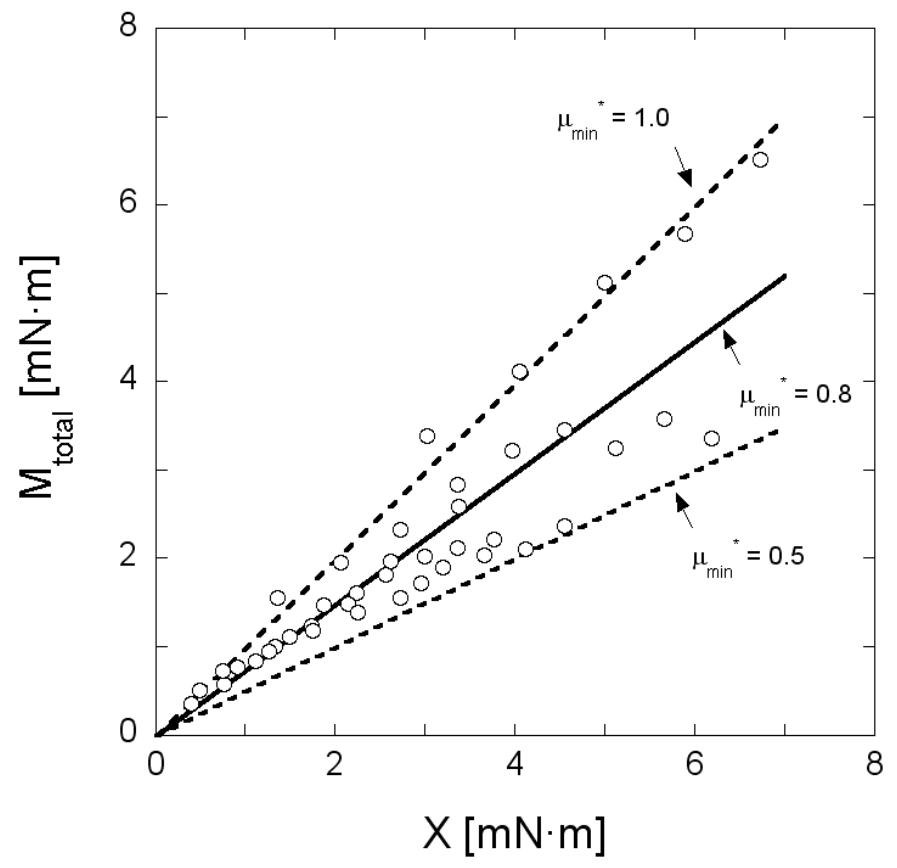

Figure 5.4. Quasi-static torque measurements presented in accordance with Eq. (5.19). Data calculated from measurements of equilibrium torque on a vane rotating at $0.03 \mathrm{rad} / \mathrm{s}$ in a $203-\mu \mathrm{m}$ glass-bead powder. Immersion depths from $10 \mathrm{~mm}$ to $90 \mathrm{~mm}$ were tested using tools of varying geometry $(R=8 \mathrm{~mm}, H=2-32 \mathrm{~mm})$. Linear regression on the entire data set yields $\mu_{\min }^{*}$ of 0.8 . The lower and upper extremes of the set are bound by $\mu_{\text {min }}^{*} 0.5$ and 1.0 , respectively.

- Region $I$ - below $\Omega$ of $0.5 \mathrm{rad} / \mathrm{s}$, the torque is independent of rotational rate for all depths. This type of flow is characteristic of the quasi-static regime where vane stresses derive from bulk friction.

- Region II - between $0.5 \mathrm{rad} / \mathrm{s}$ and $2 \mathrm{rad} / \mathrm{s}$, the torque profiles show a slight increase with rotational rate. While frictional effects still dominate, it is believed that collisional dissipation of energy yields increasing torque at higher flow rates. This increase in measured torque is more dramatic from $2 \mathrm{rad} / \mathrm{s}$ to $8 \mathrm{rad} / \mathrm{s}$, especially at deep immersion depths, where the magnitude of torque nearly triples in some cases. This region may correspond to a transitional state in the granular bed where the particles in the vicinity of the vane become partially fluidized.

- Region III - beyond $8 \mathrm{rad} / \mathrm{s}$, there is a strong moderation of the torque increase. While still significant at deep immersions, the rate of torque gain is much slower than seen in the Region II. This region may correspond to intermediate flow.

While it is speculated that the three regions outlined above correspond to granular flows spanning the quasi-static and intermediate regimes, exact comparison of the expected regimes with those observed herein is difficult, as the rate of increase in torque is highest in Region II. This contrasts with the behavior outlined in Tardos et al. (Tardos et al. 2003) where the flow exponent appears to increase monotonically over the flow regimes. 


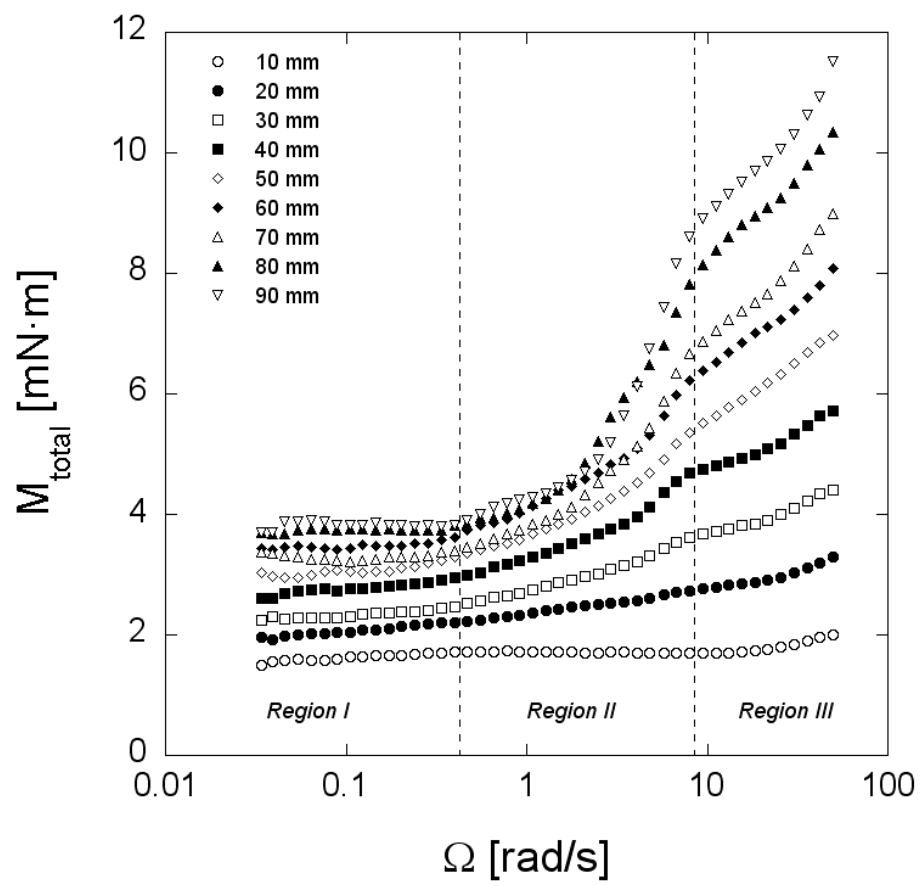

Figure 5.5. Total torque acting on an $8-\mathrm{mm} \times 16-\mathrm{mm}$ vane immersed in a $203-\mu \mathrm{m}$ glass-bead powder as a function of vane rotational rate at various immersion depths. The transition in flows that occurs from $0.03 \mathrm{rad} / \mathrm{s}$ to $50 \mathrm{rad} / \mathrm{s}$ is not smooth, but appears to occur in a number of steps. Below $0.5 \mathrm{rad} / \mathrm{s}$, the torque is independent of rotational rate. At $2 \mathrm{rad} / \mathrm{s}$, a strong increase in torque is observed. This increase becomes more moderate at $8 \mathrm{rad} / \mathrm{s}$.

Figure 5.6 shows the depth dependence of torque in the three flow regimes outlined above. Below $50 \mathrm{~mm}$, the variation of torque with immersion depth is linear in all flow regimes and appears to be consistent with the lithostatic formulation [i.e., Eqs. (5.7 to 5.10)]. At deeper immersions, negative deviations from linearity are observed. These are indicative of the stress saturation seen in Fig. 5.3. The low number of observations and the high degree of uncertainty for data below $50 \mathrm{~mm}$ in Fig. 5.3 make it difficult to evaluate whether a lithostatic portion is observed there similar to that seen in the torque profile in Fig. 5.6. The observed increase in the linear slope with $\Omega$ can be interpreted as increasing $\bar{\mu}^{*}$.

For a dynamic bulk friction coefficient of 0.8 and a container radius of $70 \mathrm{~mm}$, the depth at which stresses acting on a central vane fully couple with the container wall is $56 \mathrm{~mm}$. Figure 5.6 confirms this coupling depth, as the breakdown of linearity occurs between $50 \mathrm{~mm}$ and $60 \mathrm{~mm}$. It should be stressed that this coupling length is based on an object placed at the axis of radial symmetry, and as such, it will be smaller than the Janssen screening length, which is based on the overall diameter of the beaker. Based on the $56 \mathrm{~mm}$ coupling length, it is reasonable to assume that data collected beyond $50 \mathrm{~mm}$ will be subject to pressure saturation from wall friction and, potentially, radial confinement (normal) forces. Another observation is that the linearity of the torque profiles at immersions less than $50 \mathrm{~mm}$ and the subsequent breakdown beyond $50 \mathrm{~mm}$ are maintained over the various flow regimes. This is consistent with the observation made in Ref. (da Cruz et al. 2005) that a single dynamic bulk friction coefficient still describes the shear / normal stress relationship for sheared granular materials. 


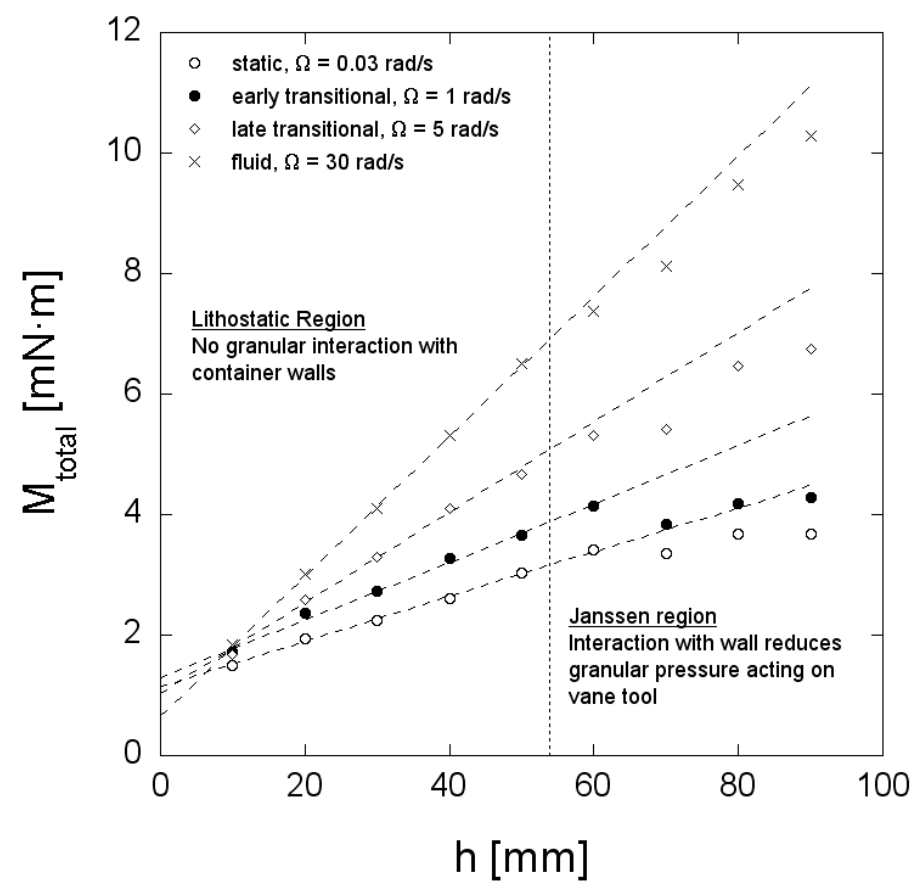

Figure 5.6. Total torque acting on a $8-\mathrm{mm} \times 16-\mathrm{mm}$ vane immersed in a $203-\mu \mathrm{m}$ glass-bead powder as a function of depth at various points in the flow regime. Profiles show lithostatic behavior up to $50 \mathrm{~mm}$. At deeper immersions, deviation from linearity results from interaction of the vane stresses with the container walls.

The origin of the three behaviors outlined in the preceding paragraphs appears to be changes in the flow dynamics at the disk and cylindrical surfaces of rotation. Figure 5.7 shows direct measurements of the shaft contributions both as a function of flow rate and as a function of depth. With regard to the rotational rate dependence, shaft torque exhibits many of the same characteristics as total torque. Low immersion depths show little increase in torque with $\Omega$. Larger depths do show some increase in torque, but only after $0.5 \mathrm{rad} / \mathrm{s}$. The data below $2 \mathrm{rad} / \mathrm{s}$ are particularly noisy for immersion depths greater than $50 \mathrm{~mm}$, suggesting that the shaft torque profile at these depths is strongly influenced by transient normal stresses and particle jamming. Comparison with Fig. 5.5 reveals that the shaft torque represents up to $25 \%$ of the total torque (at the deepest immersion depth) in the quasi-static regime. This has consequences for any subsequent analysis, as the measurement noise introduces uncertainty into the estimation of the cylinder and disk torque contributions at low rotational rates.

Figure 5.7b shows the depth dependence of shaft torque. Because the area of the shaft tool in contact with the granular material is significantly impacted by increases in immersion depth, the shaft torque scales quadratically for $h<60 \mathrm{~mm}$ as per Eq. (5.9). Significant deviation from the lithostatic pressure profile is observed at $h>50 \mathrm{~mm}$. This is consistent with the breakdown in lithostatic behavior seen in the total torque profiles; slip of granular material about the vane tool shaft, which does not have an immobilized layer of beads and is only partially roughened, may also contribute to deviation from the expected behavior. The behavior of torque with depth does not change in Region I. Increases only begin in Region II and continue into Region III.

Figure 5.8 shows the averaged disk torque contributions (from the upper and lower surfaces of vane rotation) as a function of rotation rate and depth. As described in Section 5.1.2, these contributions are determined by extrapolating the intercept of shaft-corrected torque $\left(M_{\text {total }}-\right.$ 

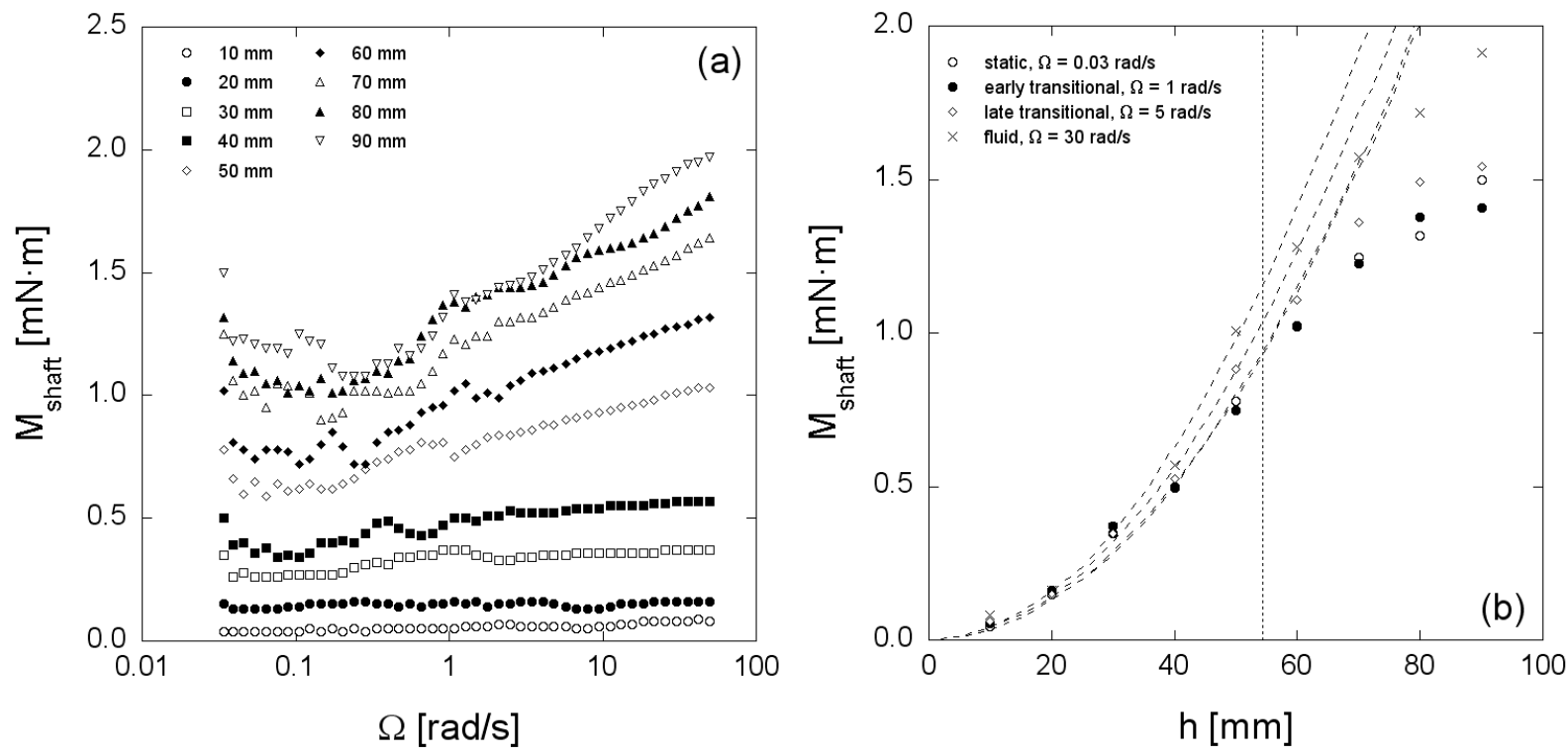

Figure 5.7. Torque contributions for a $3-\mathrm{mm}$ shaft immersed in a $203-\mu \mathrm{m}$ glass-bead powder as a function of rotational rate at various depths (a) and as a function of depth at specific points in the torque evolution (b). Similar to total torque, profiles show lithostatic behavior up to about $50 \mathrm{~mm}$ and deviate from Eq. (5.9) [quadratic dependence on $h$, represented by dashed lines] at deeper depths because of the interaction of stress chains with the container.

$M_{\text {shaft }}$ ) versus vane heights $(H)$ curves. With regard to the rotational-rate functionality, profiles for immersion depths less than $60 \mathrm{~mm}$ generally increase with increasing $\Omega$. There appears to be a slight maximum in the Region II, but this might be an artifact of the extrapolation method. The curves show the proper depth dependence, with deeper immersions resulting in higher torques.

In contrast, the profiles are not well behaved for immersions deeper than $50 \mathrm{~mm}$. A maximum occurs at low rotational rates, and significant scatter is observed in the depth dependence. The primary cause for loss of data quality at the higher flow rates is uncertainty in the vane height dependence of torque. Figure 5.9 compares vane height extrapolations at shallow and deep immersions ( $20 \mathrm{~mm}$ and $90 \mathrm{~mm}$ respectively). At low immersion depths, the torque follows the expected quadratic decrease as the vane height approaches zero. The additional data point for a vane height of $H=32 \mathrm{~mm}$ allows a reasonable estimate of the vane end effects in this case. Extrapolation at deeper immersions is complicated by the loss of the $32 \mathrm{~mm}$ measurement and point-to-point scatter. This leads to the uncertainty in the average top/bottom vane contributions, as reflected by the significant scatter at depths greater than $50 \mathrm{~mm}$ in part b of Fig. 5.8. For this reason, it is impossible to determine the nature of the deviation of stress from the lithostatic slope (i.e., the averaged change in torque with depth measured over $10 \mathrm{~mm}$ to $50 \mathrm{~mm}$ ) for the deep immersion shaft end effects.

The disk torque contributions at shallow immersion depths appear to follow a zero intercept lithostatic profile beyond Region I. An unexpected observation is that the averaged vane end effects in Region II and III all appear to follow the same linear (lithostatic) trend, regardless of rotational rate. Figure 5.10 shows the analysis of the lithostatic slope of the vane end torque for immersion depths below $60 \mathrm{~mm}$. At rotational rates greater than $1 \mathrm{rad} / \mathrm{s}$, the lithostatic slope approaches a constant value. This limiting value is consistent with torque calculations for a Coulombic solid with a lithostatic normal stress distribution and a uniform bulk friction 

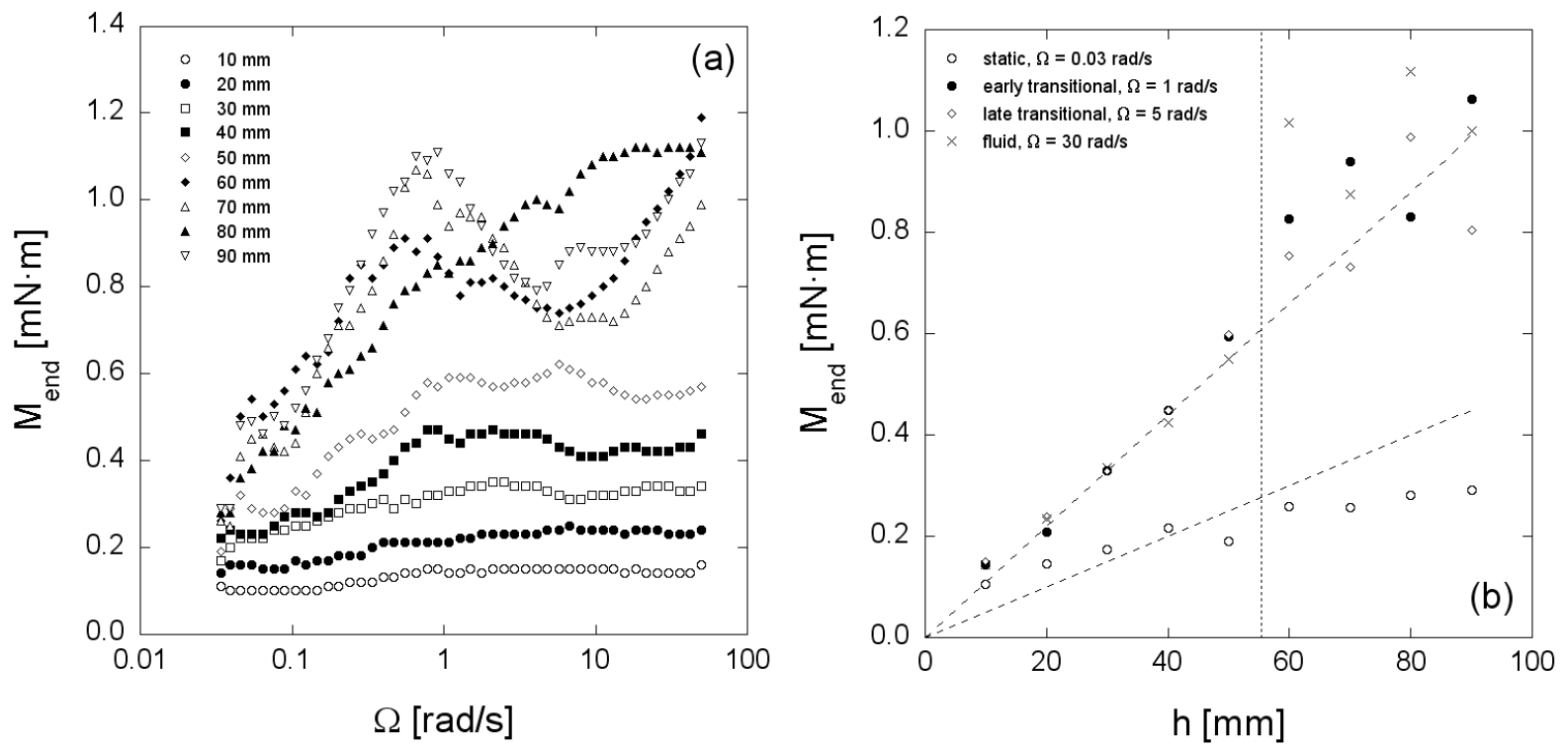

Figure 5.8. Averaged disk torque contributions for an $8-\mathrm{mm} \times 16-\mathrm{mm}$ vane immersed in a 203- $\mu \mathrm{m}$ glass-bead powder as a function of rotational rate at various depths (a) and as a function of depth at various flow regimes (b). Dashed lines illustrate the linear / zero intercept dependence of torque on $h$ [Eq. (5.15)].
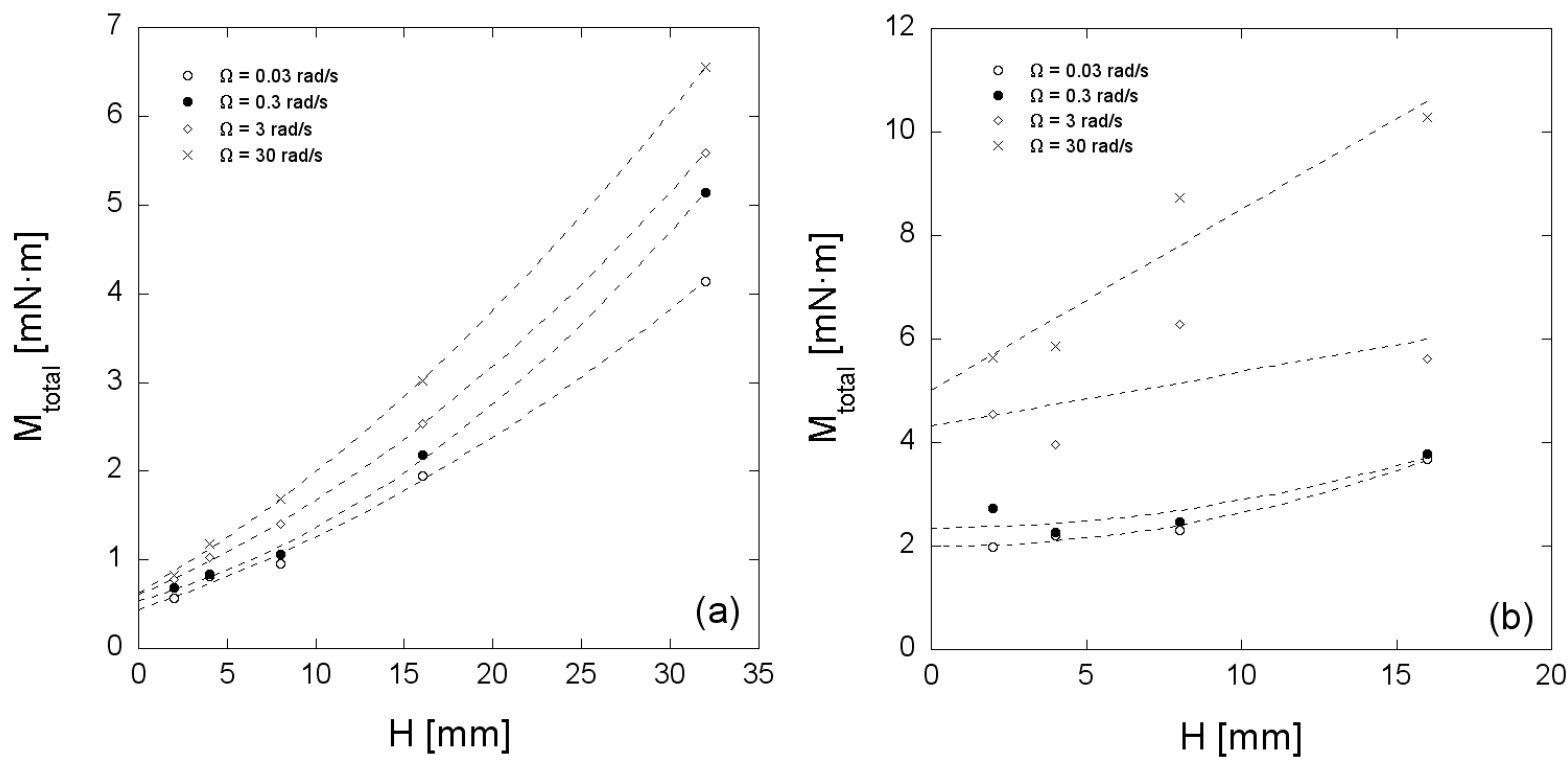

Figure 5.9. Total vane torque as a function of vane height at an immersion depth of $20 \mathrm{~mm}$ (a) and $90 \mathrm{~mm}$ (b). Dashed curves are the best-fit lines used to determine vane end effects. Extrapolations at low vane immersion depths follow the expected quadratic functionality [Eq. (5.10)] while extrapolations at deeper immersion depth are complicated by significant point-to-point scatter. 
coefficient equal to 0.8 (i.e., equal to $\mu_{\text {min }}^{*}$ ). This result indicates that granular interactions at the vane ends are purely Coulombic above $1 \mathrm{rad} / \mathrm{s}$ and that flow effects observed in total torque profiles for $\Omega>1 \mathrm{rad} / \mathrm{s}$ must derive primary from the vane radial surfaces.

On the other hand, lower-than-expected top/bottom torque contributions are seen at rotational rates below $1 \mathrm{rad} / \mathrm{s}$. This negative deviation may derive in part from the scatter in the shaft and total torque measurements in the quasi-static regime, or it may suggest that the granular stress network is altered in the presence of the vane tool, which can support part of the vertical weight through frictional interaction with the particles. Particle bridging and jamming effects may also contribute to the flatness of the torques in this region.

Figure 5.11 shows the cylindrical (side) contributions acting on the vane tool, both as a function of rotation rate at various depths and as a function of depth at select points in the flow curve. No significant increase in cylindrical torque is observed over Region I. Indeed, most of the change occurs across Regions II and III. This contrasts with the disk torque profiles where most of the change occurs in Region I. The depth dependence generally follows the expected trends, with deeper immersions yielding higher cylinder torque.

In Region I, the lithostatic slope is very close to zero. This observation is difficult to rationalize in terms of granular medium statics, as zero lithostatic slope only occurs in isotropic media like colloidal suspensions and polymer gels. One possible cause is the transfer of part of the lithostatic loading onto the vane tool itself. At higher rotational rates, the observed increase in lithostatic slope is indicative of flow effects. As before, deviation from linearity is observed at immersions deeper than $50 \mathrm{~mm}$. Here, significant deviations are observed at the beginning of the Region II and yield a minimum in the torque versus rotational rate curve for $70 \mathrm{~mm}$ to

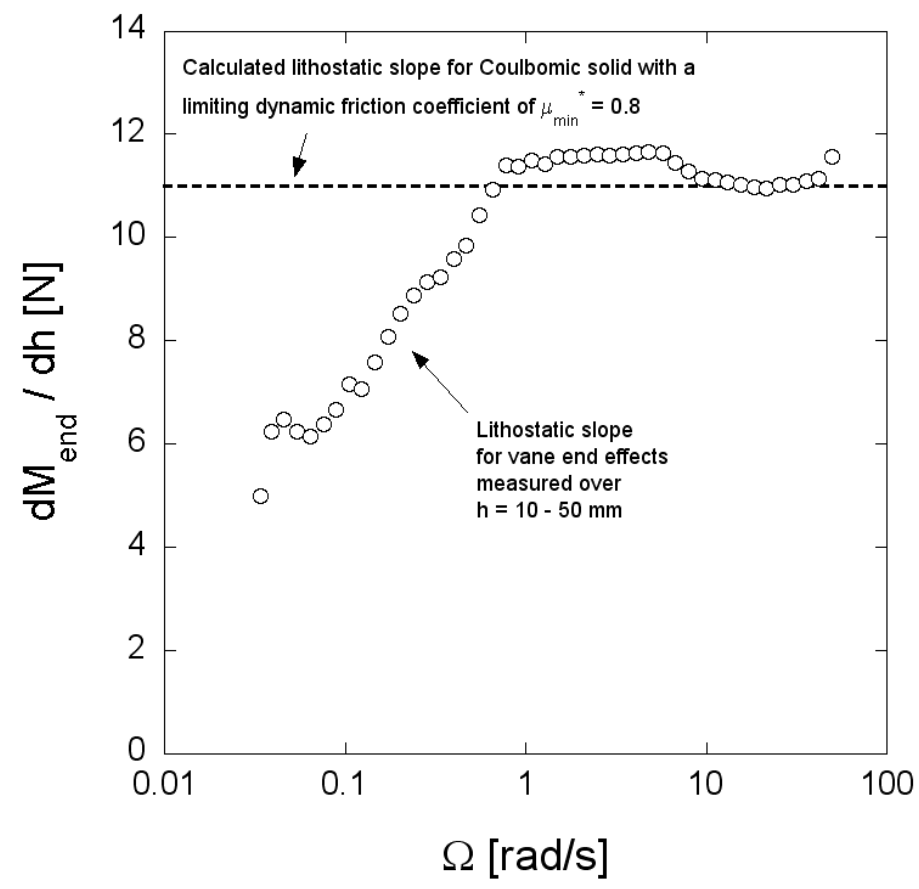

Figure 5.10. Lithostatic slope for vane end effects from $10 \mathrm{~mm}$ to $50 \mathrm{~mm}$ as a function of rotational rate. Beyond $1 \mathrm{rad} / \mathrm{s}$, the lithostatic slope appears to be independent of rotational rate and behaves as a Coulomb solid with a bulk friction coefficient equal to the limit of kinetic friction at zero flow (i.e., $\mu_{\min }^{*}=0.8$; dashed line). 
$90 \mathrm{~mm}$. It is speculated that the primary contributor to the minimum is the error in the disk torque extrapolations at deep immersion depths and that improvement could be achieved by more rigorous end-effect analysis; however, some granular rheology studies have also found evidence of torque decreases with increasing rotational rate (Kuno and Kurihara 1965, Kuno and Senna 1967).

\subsubsection{Prediction of Vane Rheograms for the Dry Powder}

The observations made in the preceding sections provide a semi-empirical basis by which vane rheograms for cohesionless granular materials can be interpreted. The observed behaviors are complex functions of flow rate and immersion depth. At low rotational rates, decoupling of the torque contributions yields unexpected results, such as the negative deviations from Coulombic behavior seen on the disk surfaces of the vane and the zero lithostatic slope observed on the rotating cylinder surfaces. These unexpected observations may result partly from interaction of the continuous particle contact network with the vane tool. While these complexities make the analysis of decoupled torque contributions ambiguous, they do not prevent analysis of the total torque profile in this region. Indeed, the behavior observed in the total torque at rotational rates below $0.5 \mathrm{rad} / \mathrm{s}$ appears quasi-static, and as such, the analysis presented in Section 5.3.1 provides a reasonable approach to characterizing vane rheograms for $\Omega<0.5 \mathrm{rad} / \mathrm{s}$ (see Fig. 5.4). Equations (5.19) and (5.20), which are based on the approach outlined in Ref (Poloski et al. 2006) and are similar in form to granular torque expressions in Ref (Benarie 1961), allow frictional characterization of the $203-\mu \mathrm{m}$ powder to $25 \%$ accuracy using a single quasi-static descriptor, $\mu_{\text {min }}^{*}$. Breakdown of the linearity in the total torque profiles beyond $50 \mathrm{~mm}$ (Fig. 5.6) confirms the appropriateness of the Janssen normal stress distribution used in the quasi-static analysis.

At the highest rotational rates, successful decoupling of the torque profiles provides a relatively unhindered view of the flow dynamics occurring at the disk, cylindrical surfaces. The disk torque is well described by a single lithostatic slope using a bulk friction coefficient equivalent to
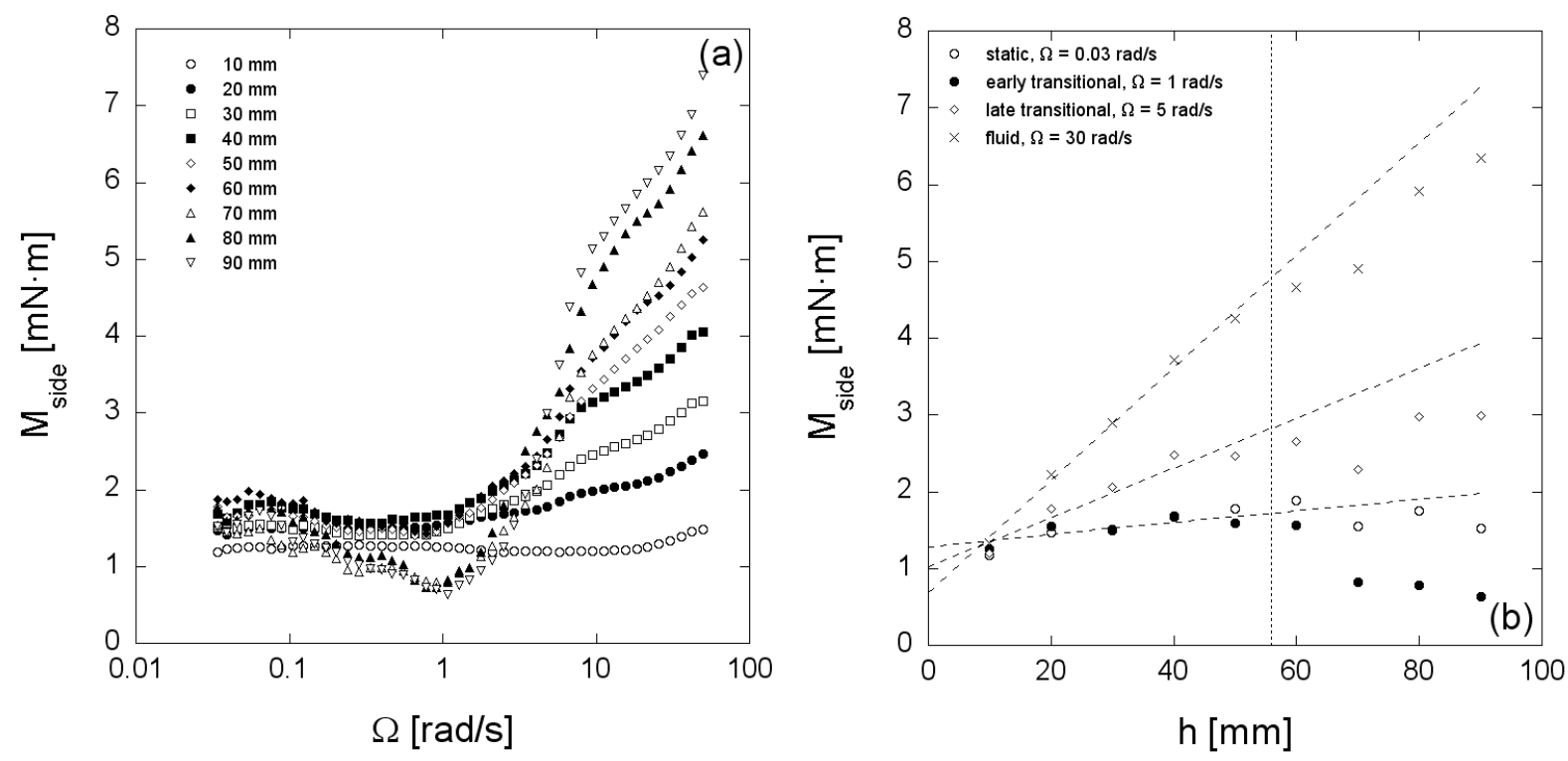

Figure 5.11. Cylindrical (side) torque contributions for an $8-\mathrm{mm} \times 16-\mathrm{mm}$ vane immersed in a 203- $\mu \mathrm{m}$ glass bead powder as a function of rotational rate at various depths (a) and as a function of depth at various flow regimes (b). Dashed lines illustrate the linear dependence of torque on $h$ [Eq. (5.10)]. 
$\mu_{\text {min }}^{*}(0.8)$ at rotational rates above $1 \mathrm{rad} / \mathrm{s}$. Although the data beyond $50 \mathrm{~mm}$ are scattered, the same lithostatic slope as used for sub-50-mm measurements provides a reasonable approximation of the system. The assumption of Coulombic interactions allows the vane torque to be predicted from the granular matter properties determined above. The exact expression for bottom and top disk torque contributions is given by the sum of Eqs. (5.7) and (5.8), with the apparent bulk friction coefficient, $\bar{\mu}^{*}$, set to $\mu_{m i n}^{*}$,

$$
M_{\text {end }, \text { pred }}=\frac{2 \pi}{3} \mu_{\text {min }}^{*} \mathrm{v}_{p} g\left[R^{3}(H+2 h)-R_{s}^{3} h\right]
$$

Unlike the disk surfaces, the radial surfaces of the vane (including both the cylinder and the shaft) show dynamic flow effects and cannot be properly modeled using only Coulombic equations. Instead, the granular flow model adapted from da Cruz et al. (see Ref. (Sáez et al. 2006)) will be used to predict the flow behavior on both the shaft and cylinder of rotation. Because no direct measurements of solids fraction were made, it shall be considered uniform, and the dilation law will be ignored. Under these circumstances, the radial shear stress acting on the tool at a particular depth $z, \tau_{v}$, can be determined from granular properties and rotational rate by combining Eqs. (4.2) and (4.3) and taking $P=P_{r r}$. Together with Eq. (5.3), this yields

$$
\tau_{r \theta}=\mu_{m i n}^{*} K P_{z z}+b d \sqrt{\rho_{p} K P_{z z}} \dot{\gamma}_{r \theta}
$$

To be consistent with the sign conventions established above, $\dot{\gamma}=-r(\partial \omega / \partial r)$, where $\omega=v_{\theta} / r$. For the radial surfaces of rotation, the $\theta$ momentum balance in the formulation of Sáez et al. reduces to

$$
\tau_{r \theta}=\left(\frac{R}{r}\right)^{2} \tau_{v}
$$

Substitution of this expression into Eq. (5.22) and subsequent integration over the zone of sheared material, which can be expressed as the region between the vane radius $R$ and the radial extent of shear $R_{o}=R \sqrt{\tau_{v} /\left(\mu_{\text {min }}^{*} K P_{z z}\right)}$, yields, after some algebraic manipulation, an implicit relation for $\tau_{v}$. It is,

$$
\tau_{v}=\mu_{m i n}^{*} K P_{z z} \ln \left(\frac{\tau_{v}}{\mu_{m i n}^{*} K P_{z z}}\right)+\mu_{m i n}^{*} K P_{z z}+2 b d \sqrt{\rho_{p} K P_{z z}} \Omega
$$

Because $\mu_{\text {min }}^{*}, K$, and $\mu_{w}$ have been determined independently, Eq. (5.24) has a single adjustable parameter: the da Cruz constant $b$, which determines the rate of stress increase with increasing $\Omega$. To determine the cylinder and shaft torque contribution, $\tau_{v}$ must be determined and subse- 
quently integrated over the vane length:

$$
\begin{gathered}
M_{\text {side,pred }}=2 \pi R^{2} \int_{h}^{h+H} \tau_{v}(z) d z \\
M_{\text {shaft,pred }}=2 \pi R_{s}^{2} \int_{0}^{h} \tau_{v}(z) d z
\end{gathered}
$$

As before, the total vane torque is determined by summing the predicted contributions of the shaft, disk, and cylinder contributions.

The approach outlined above allows for the calculation of total vane torque for what appear to be quasi-static and intermediate flows. Figure 5.12 shows fits of the model to Regions I and III. Below $0.5 \mathrm{rad} / \mathrm{s}$, torque profiles, shown by solid lines, are calculated using Coulombic equations with a Janssen pressure distribution. The theory tends to over-predict the increase in torque with vane immersion depth observed experimentally; it is speculated that this is an artifact of using $K$ and $\mu_{w}$ determined from static radial stress distributions. Including stress distributions measured under dynamic conditions may alleviate this problem. Above $10 \mathrm{rad} / \mathrm{s}$, the torque profiles are calculated from Eqs. (5.21 to 5.26) using a lithostatic pressure profile ( $b=5$ for all depths). Here, quantitative fits of the data are obtained, with the difference between experimental and predicted values typically less than $10 \%$ at $\Omega>10 \mathrm{rad} / \mathrm{s}$.

Torque calculations at high rotational rates could have been based on Janssen's equation; however, to predict the correct magnitude of torque increase in Region III, $b=15$ must be employed.

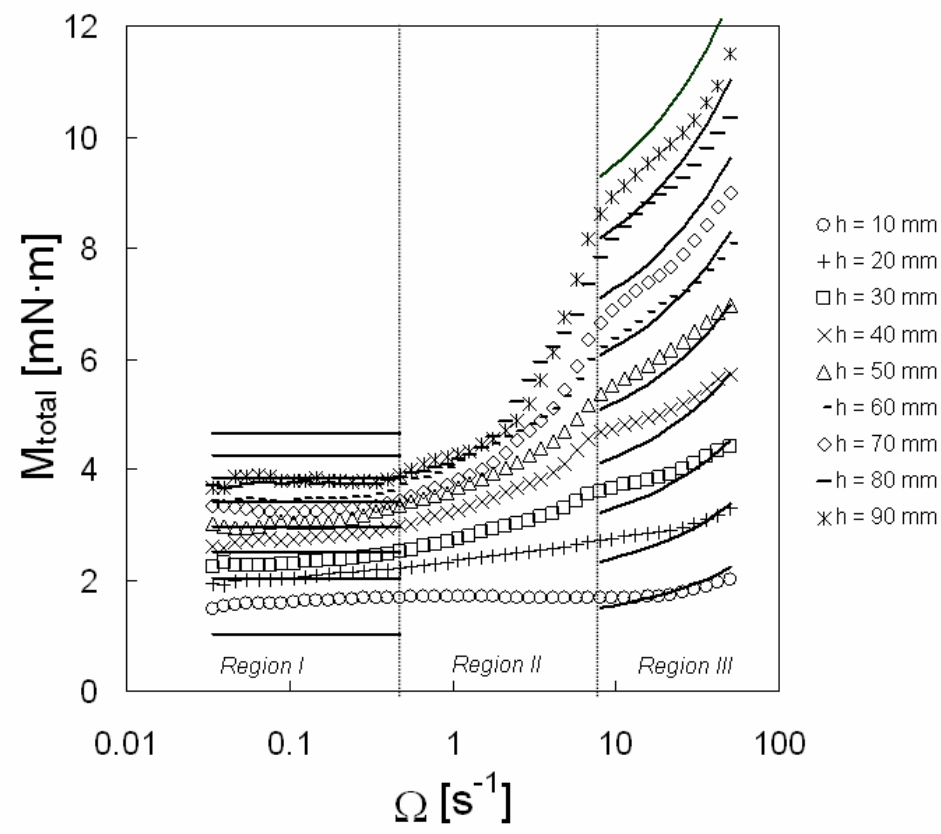

Figure 5.12. Comparison total vane torque for the $203-\mu \mathrm{m}$ glass bead powder at various immersion depths with predictions (solid lines) based on a Janssen stress profile [Eq. (5.1)] for $\Omega<0.5 \mathrm{rad} / \mathrm{s}$ and on a lithostatic stress profile [Eq. (5.4)] for $\Omega>8 \mathrm{rad} / \mathrm{s}\left(K=0.6, \mu_{w}=0.7\right)$. 
The resulting profiles do not capture either the quantitative or qualitative behavior. Specifically, the slope of the torque profiles is too severe, as shown in Fig 5.13. This could be corrected by lowering $b$; however, the saturation of the pressure profile would cause the curves to under-predict torque at deep immersions. As such, no parametric variation of the Janssen-based calculations describes the torque behavior at high flow rates.

It should be stressed that predictions based on Eq. (5.24) are constrained by inertial requirements. Specifically, the linear limit of the friction law postulated by da Cruz is $I \approx 0.20$, beyond which bulk friction becomes insensitive to changes in the inertial number. The radial surface contribution predictions are all based on a friction coefficient that varies linearly with $I$. While the $I=0.20$ limit only applies (strictly) to two-dimensional simulations, it would still be useful to confirm that the predictions made herein meet this requirement. Calculating $I$ reveals that all profiles satisfy $I<0.20$ except for the last few data points at $10 \mathrm{~mm}$.

The results in Fig. 5.12 show that semi-quantitative characterization of granular flows is possible in Regions I and III. It can be speculated that the distribution of granular stresses influencing vane rheology is sufficiently stable to allow Janssen and lithostatic profiles to be used for low and high rotational rates, respectively. Slow rotation of the vane still allows for long particle contact and does not alter the microstructure. In this case, the static Janssen parameters provide a reasonable estimate of the system. A fast vane rotation increases particle separation, eliminating both frictional contacts and the continuous stress network. This, in turn, alters the stress distribution from one described by a Janssen profile to one that approaches a lithostatic profile. In the limit of complete bed fluidization, a lithostatic pressure profile would be expected for reasons given in Iverson and Vallance (Iverson and Vallance 2001).

The transition from Janssen-based predictions that slightly over-predict torque gains at low rota-

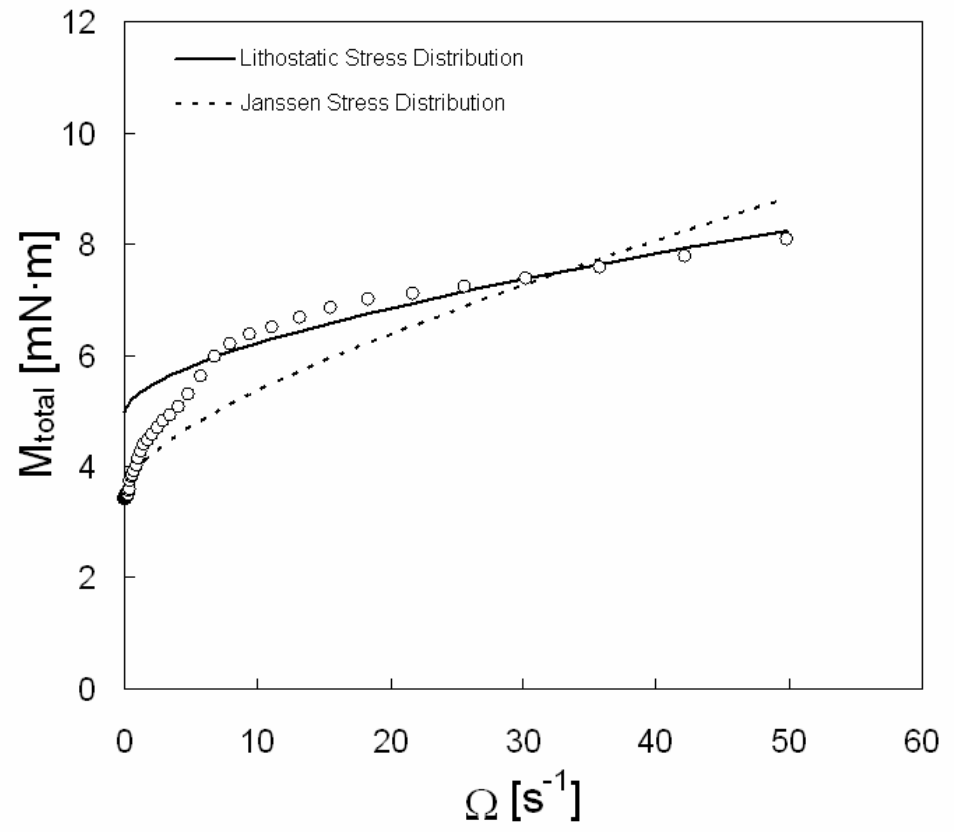

Figure 5.13. Comparison of total torque predictions at high rotational rates against experimental data for $60 \mathrm{~mm}$. Torque calculations based on a lithostatic pressure distribution (solid line, $b=5$ ) provides a better representation of the data than calculations based on a Janssen pressure distribution (dashed line, $b=15$ ). All fits use $K=0.6, \mu_{w}=0.7$, and $\mu_{\min }^{*}=0.8$. 
tional rates (suggesting significant particle contact networks) to those that highly over-predict increases at high rotational rates is indicative of fluidization of the material in the vicinity of the vane. With regard to Region II, the result of increased particle separation is that the microstructure is sufficiently altered so that the static Janssen profile no longer holds. While the approach of Sáez et al. (Sáez et al. 2006) accounts for fluidization in the formulation of the bulk friction coefficient, it does not allow for changes in the stress distribution due to elimination of stress coupling with the container walls, and as such, cannot accurately predict the "transitional" flows observed here. That being said, the torque in Region II could be predicted by empirically incorporating rotational rate dependence into the Janssen stress-distribution profile. The most appropriate way to do this would be to make $\lambda$ a function of $\Omega$, such that $\lambda \rightarrow \infty$ as the powder is fully fluidized by vane rotation; however, this is beyond the scope of this report.

\subsubsection{Prediction of Vane Rheograms for Glass Bead Slurries}

The dry glass bead flow curve studies should provide a basis by which more complex systems may be characterized. To verify this, the methods and theory outlined above were applied to the study of vane rheograms of a dense $203-\mu \mathrm{m}$ glass bead slurry in water. The only major difference between modeling the dry powder and the slurry is that the slurry pressure distributions, Eqs. (5.1) and (5.4), employ a buoyant material density, $\rho_{b}$, in place of the particle density. The buoyant density is defined as

$$
\rho_{b}=\rho_{p}-\rho_{\text {sus }}
$$

where $\rho_{\text {sus }}$ is the density of the suspending phase (in this case, water: $\rho_{\text {sus }}=1 \mathrm{~g} / \mathrm{cm}^{3}$. Figure 5.14 shows the direct characterization of the granular properties, including $\mu_{\text {min }}^{*}, K$, and $\mu_{w}$, from kinetic shear strength data. Fits of the quasi-static torque data at $\Omega=0.03$ rads using Eqs. (5.19) and (5.20) find $\mu_{\text {min }}^{*}=0.55, K=0.6$, and $\mu_{w}=0.55$. Figure 5.15 shows vane rheograms for the slurry spanning immersion depths from $10 \mathrm{~mm}$ to $90 \mathrm{~mm}$. It shows the same general trends as the dry glass bead powder. Specifically, a quasi-static region is observed at low rotation rates, and significant dynamic torque increases are observed at rotational rates greater than $1 \mathrm{rad} / \mathrm{s}$. At all rotational rates, torque generally increases with increasing immersion depth. In contrast to the dry bead results, there appears to be a brief plateau in the depth dependence around $60 \mathrm{~mm}$ (see Fig. 5.14). The origin of this plateau is unknown, but it may be a container effect resulting from coupling of the vane stress fields with the wall.

Based on the dry glass bead results, torque curves are considered quasi-static below rotation rates of $0.5 \mathrm{rad} / \mathrm{s}$ and intermediate above $8 \mathrm{rad} / \mathrm{s}$. The solid curves shown in Fig. $5.15 \mathrm{repre-}$ sent predictions based on the vane model developed in Section 5.3.3. After the dry glass bead predictions, calculations for the quasi-static region assume a Janssen-type pressure distribution. Pressure distributions in the intermediate region are considered lithostatic. Calculations in the intermediate region employ a da Cruz friction law constant of 8 , which provides an adequate reproduction of the experimental data.

Application of the model to the slurry system confirms the transition in pressure distribution observed in the dry glass bead powder. At low rotational rates, the torque profiles show the influence of pressure saturation, suggesting a Janssen normal stress distribution. This transitions to torque profiles that are better described using a lithostatic pressure profiles.

With regard to the fitting parameters used to model the glass bead slurry, two noteworthy obser- 


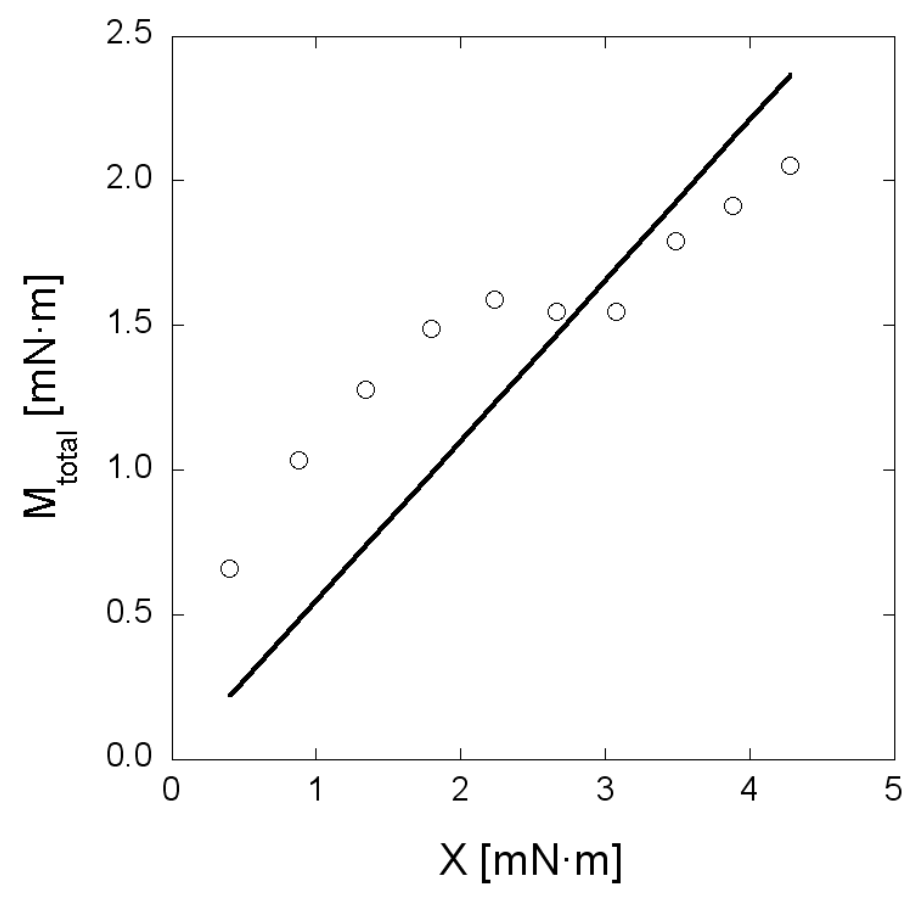

Figure 5.14. Quasi-static torque measurements for the glass bead slurry presented in accordance with Eq. (5.19). Data are calculated from measurements of equilibrium torque on a vane rotating at $0.03 \mathrm{rad} / \mathrm{s}$ in a $203-\mu \mathrm{m}$ glass-bead slurry in water. Immersion depths from $10 \mathrm{~mm}$ to $90 \mathrm{~mm}$ were tested using an $8-\mathrm{mm} \times 16-\mathrm{mm}$ vane tool. Analysis (solid curve) of the data yields $\mu_{\text {min }}^{*}=0.55, K=0.6$, and $\mu_{w}=0.55$.

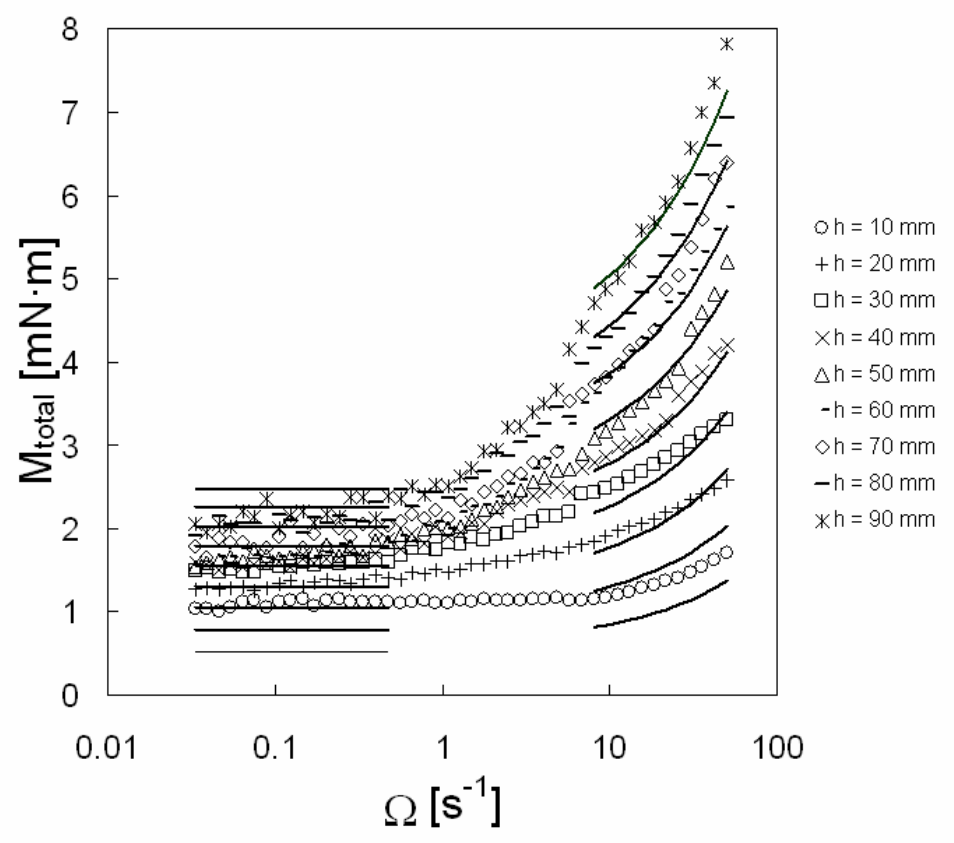

Figure 5.15. Comparison total vane torque for the $203-\mu \mathrm{m}$ glass bead slurry at various immersion depths with predictions (solid lines) based on a Janssen stress profile [Eq. (5.1)] for $\Omega<0.5 \mathrm{rad} / \mathrm{s}$ and on a lithostatic stress profile [Eq. (5.4)] for $\Omega>8 \mathrm{rad} / \mathrm{s}\left(K=0.6, \mu_{w}=0.7\right)$. 
vations should be made. First the limiting friction coefficient, $\mu_{m i n}^{*}$, for the slurry is lower than that of the dry glass bead powder $(0.6$ wet versus 0.8 dry). This is indicative of lubrication of the particle contacts by the suspending phase. Secondly the da Cruz friction law constant, $b$, has increased from 5 for the dry powder to 8 for the slurry. This increase, and the relative increase in torque it causes, may result from increased dissipation of particle energy by viscous drag around the particle, which is expected to be significantly increased in water over air.

In its current state, the model and analysis presented herein is capable of characterizing vane data at low and high rotational rates. When applied to the analysis of both dry glass bead powders and glass bead slurries in water, appropriate descriptors for the granular properties, namely $K$, $\mu_{w}$, and $\mu_{\text {min }}^{*}$, can be measured explicitly or inferred as in Ref. (Poloski et al. 2006). The da Cruz friction law constant, $b$, can be determined by comparing the experimental rheograms to the total torque.

\subsection{Conclusions}

In this chapter, the rheology of a cohesionless powder comprised of 203- $\mu \mathrm{m}$ glass spheres is characterized using an $8-\mathrm{mm} \times 16-\mathrm{mm}$ (radius by height) shear vane tool. As expected, the torque profile (i.e., vane rheogram) for this powder is a complex function of both immersion depth and vane rotational rate. Increased vane immersion depth always results in increased torque. In the most general terms, increased rotational rate is observed to increase vane torque. However, the range of rotational rates studied is sufficiently broad to encompass several different flow behaviors. Three separate flow regions spanning quasi-static and intermediate flow regimes are identified. By breaking down the total torque acting on the vane tool into contributions from the shaft, disk, and cylinder, the origin of the three is identified as transitions in flow behavior on different surfaces of the vane tool.

On the cylindrical surfaces of rotation, dynamic increases in torque typically occur for rotational rates greater than $2 \mathrm{rad} / \mathrm{s}$. Both shaft and cylindrical torque profiles show similar qualitative trends. This similarity may result from the fact that both surfaces are normal to the axis of vane rotation. In comparison, the disk surfaces show a separate variation of torque with rotational rate with most of the dynamic response occurring at low rotational rates $(0.03$ to $0.5 \mathrm{rad} / \mathrm{s})$ The lithostatic slope for the disks of rotation saturates near intermediate flow rates $(0.5$ to $8 \mathrm{rad} / \mathrm{s})$ and does not change at higher flow rates. Analysis of the depth dependence exhibited by the disk torque profiles at rotational rates greater than $1 \mathrm{rad} / \mathrm{s}$ indicates Coulombic dynamics characterized by an effective macroscopic coefficient of friction equivalent to $\mu_{\min }^{*}(0.8)$. Thus, while the overall flow acting on the vane tool may appear to span the quasi-static and intermediate regimes, the flow dynamics acting on the disks are truly quasi-static over most of the rotational rates studied. From this observation, it may be inferred that nearly all of the dynamic effects seen in the torque profiles are a result of cylindrical torque contributions.

It is found that semi-quantitative prediction of the vane torque profiles is feasible at rotational rates below $0.5 \mathrm{rad} / \mathrm{s}$ and at those above $10 \mathrm{rad} / \mathrm{s}$. A lithostatic pressure profile yields the best prediction of torque dynamics at high rotational rates, indicating particle fluidization of the glass beads surrounding the vane. At low rotational rates, a Janssen pressure distribution appears to best capture the torque behavior with depth. The Janssen profile tends to over-predict torque at low rotational rates; however, this is most likely caused by assuming a static bed stress distribution. Predicting the vane torque profile at rotational rates between $0.5 \mathrm{rad} / \mathrm{s}$ and $10 \mathrm{rad} / \mathrm{s}$ appears to require knowledge of how the microstructure of the bed changes under the influence of vane rotation and vibration. One potential way of doing this is to have a pressure saturation length, $\lambda$, that is a function of rotational rate. 
The experimental methodology developed for the dry glass bead powder is then used to characterize the vane rheology of a slurry of $203-\mu \mathrm{m}$ glass beads in water. The transition of the pressure distribution from a Janssen-type to a lithostatic curve is confirmed. The model allows adequate prediction of the slurry rheograms at rotational rates below $0.5 \mathrm{rad} / \mathrm{s}$ and at those above $10 \mathrm{rad} / \mathrm{s}$. Again, prediction at intermediate rotational rates appears to require knowledge of the stress transition.

Overall, semi-quantitative analysis and characterization of granular materials may be accomplished using a vane rheometer under quasi-static and intermediate flow conditions. The main difficulties occur in regions where particles begin to fluidize. Direct measurements of pressure acting on the vane tool or of the three-dimensional granular packing structure (using MRI) might provide additional insight into the processes affecting vane stress over the entire range of intermediate behavior. In terms of characterizing Hanford TRU sludges, this work provides a way of correlating frictional particle interactions using common bench-scale rheometric operations. This ability to characterize the frictional properties of slurry systems, when combined with the continuum model discussed in Chapter 4 of this report, can facilitate prediction of velocity and stress profiles that exist in process equipment such as mechanical agitators. Indeed, the careful consideration of stress and flow profiles at each of the surfaces of rotation associated with the vane rheometer elucidate some of the challenges process engineers will face when working with equipment handling Hanford TRU sludge. Not only must stress anisotropy be considered, but flow anisotropy, that is, different flow regimes occurring at each of the surfaces of rotation, should be considered as well. As such, the studies presented in this chapter help improve understanding of potential issues associated with the transport, packing, and treatment of Hanford TRU sludges. 


\subsection{Anisotropic Deformation of Colloidal Particles by Ion Irradiation}

As discussed in Chapter 2, shape analysis of the undissolved solid particulates present in Hanford TRU tank sludges indicate that the particles are anistropic. Recently published data indicate that colloidal particles can deform under the influence of ion irradiation (van Dillen 2004). The data presented in this reference show the formation of oblate spheroid silica $\left(\mathrm{SiO}_{2}\right)$ and titania $\left(\mathrm{TiO}_{2}\right)$ particle under Xenon (Xe) irradiation. van Dillen (2004) developed a model for this deformation based on the electronic stopping of the ions in the colloid particles. In the immediate vicinity of the ion path, the colloid melts and solidifies on a time scale of nanoseconds. This melting and solidification process creates a cumulative stress on the colloid particle that deforms the particle over time. For TRU tank sludges, a high degree of $\alpha$ radiation may provide a similar mechanism for deformation. Using the published model with ${ }^{238} \mathrm{Pu} \alpha$ radiation rather than $\mathrm{Xe}$ ion irradiation, a fluence of approximately $1 \times 10^{15} \mathrm{ions} / \mathrm{cm}^{2}$ would be required before significant deformation is observed. Considering the relatively high degree of TRU isotopes in the sludge and the creation date of these wastes (1940s-1950s), fluences of this magnitude are expected in the actual waste and provide a potential mechanism for the creation of oblate spheroids in the TRU sludge.

This chapter presents a brief study of anisotropic deformation for select mineral solids present in Hanford TRU tank slurries. The primary goal was to determine if deformation processes similar to those observed for colloidal silica and titania occur in mineral solids that are abundant in Hanford TRU tank sludges.

\subsection{Background}

Irradiation of mineral solid colloids with heavy ions has been shown to cause anisotropic deformation provided the ion has sufficient energy to melt the material along its track. The phenomenon of induced particle deformation has been discussed in a number of previous works (Velikov et al. 2002, van Dillen 2004, Jagielski et al. 2004, Gentils et al. 2004). The basic controlled experiment by which colloids are deformed begins with the generation of an high energy ion beam from a source external to the colloid (typically using a particle accelerator). The ions comprising the beam will deposit energy into any material with which they interact depending on its density. As such, experiments are typically run under vacuum to prevent loss of beam intensity as it travels to the sample. When the ion beam encounters the colloid, it can either penetrate it completelly (i.e., travel all the way through the colloid) or be stopped by the dense medium. As the ion travels through the colloid, part or all of its energy can be transferred to the material, causing localized thermal tracks along the ions path. At sufficiently high deposition energies, the material melts and re-solidifies on the order of nanoseconds. This melting and solidification process creates a cumulative stress that deforms the colloid particle over time. A diagram outlining the anisotropic shape change of a spherical colloidal particle upon irradiation is shown in Fig. 6.1. Deformation is uniform such that a sphere deforms into an oblate spheroid, with the short axis in the direction of the ion source.

Anisotropic deformation is subject to a number of conditions. First, the ion source must be directional. As in the example shown in Fig. 6.1, particles expand along the plane perpendicular to the ion track. If the ion source were distributed uniformly around the colloid and the direction of ion tracks through the particle random, a change in particle shape would not be effected. The second condition is that the ion must have sufficient energy to penetrate the entire colloid. Low energy ions or ions interacting with large colloids may only penetrate sub-surface layers of the 


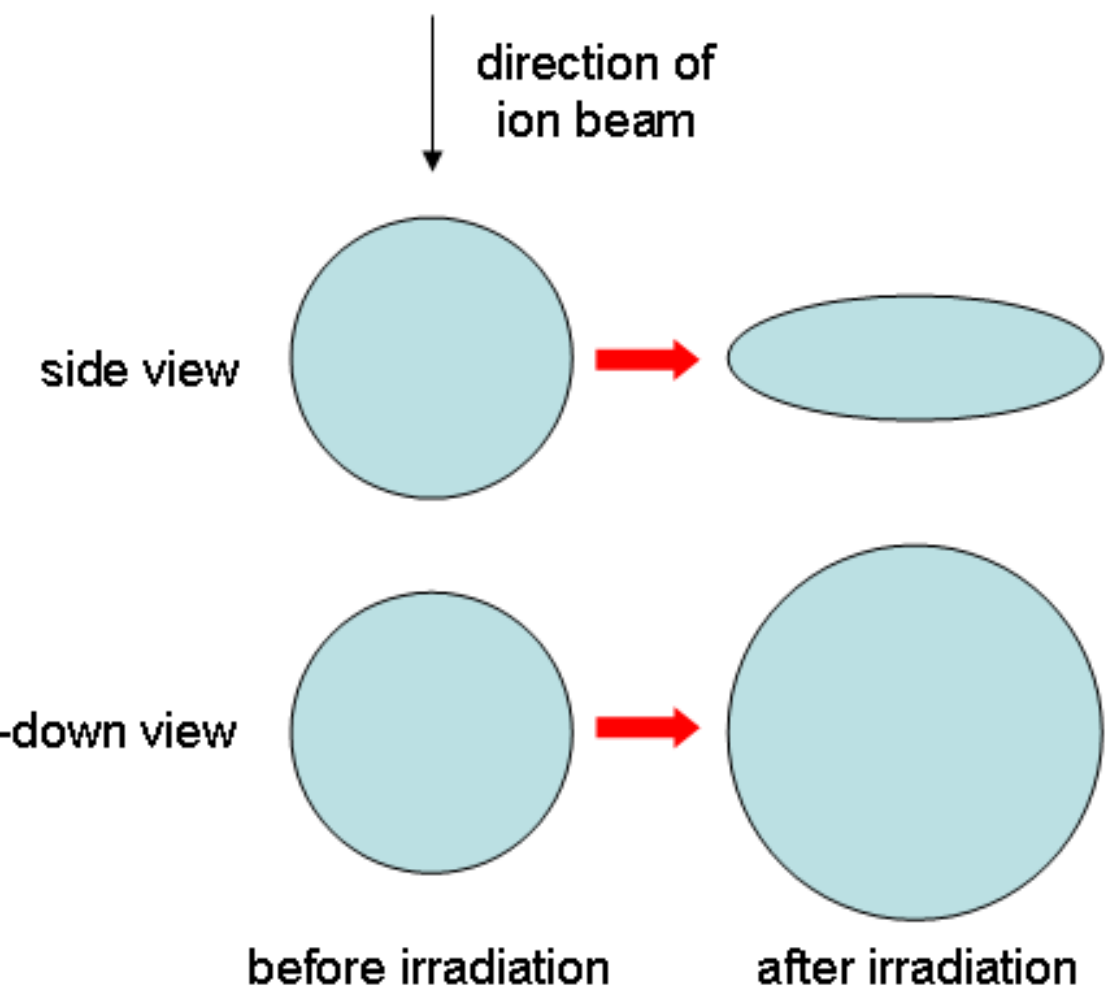

Figure 6.1. Basic illustration of the anisotropic shape change experienced by a spherical colloid undergoing ion irradiation. Here, the ion beam strikes the particle from the top, causing it to expand laterally and contract on the axis parallel to the ion beam.

material before stopping. The associated thermal spike will not penetrate the entire sample, resulting in little or no deformation. Finally, deformation results from the cumulative effect of ion tracks, as represented by the total fluence.

The goal of the study presented herein was to determine if anisotropic deformation affected select mineral solids found in Hanford TRU sludges. To this end, colloidal silica $\left(\mathrm{SiO}_{2}\right)$ and bismuth oxide $\left(\mathrm{Bi}_{2} \mathrm{O}_{3}\right)$ were irradiated with $\mathrm{Xe}^{+}$ions. Additional experiments were carried where colloid silica was irradiated with $\mathrm{He}^{+}$ions to simulate damage by $\alpha$ radiation. Any resulting deformation (or lack thereof) was recorded using a transmission electron microscope (TEM). Silica was selected based on its used in previous studies of anisotropic deformation (e.g., van Dillen (2004)). Bismuth oxide was selected for irradiation based on their presence in Hanford TRU sludges (Tingey 2004, Rapko and Lumetta 2005).

\subsection{Methods and Materials}

A silica sol was purchased from Microspheres-Nanospheres (Mahopac, New York 10541). The sol consisted of roughly mono-disperse spherical silica (average particle diameter of $50 \mathrm{~nm}$ ) suspended in a methanol-ammonia solution. To explore the effects of particle size on the radiation induced deformation process, a second mono-disperse spherical colloidal silica with a larger particle diameter was purchased from Fiber Optic Center, Inc. (New Bedford, MA 02740). This silica was supplied as a dry powder with a nominal particle diameter of $100 \mathrm{~nm}$. The material density of both silicas was approximately $2.0 \mathrm{~g} / \mathrm{cm}^{3}$. Bismuth oxide was purchased from Nanophase Technologies (Romeoville, IL 60446). It was NanoArc ${ }^{\circledR}$ Bismuth Oxide powder (average diameter: $40 \mathrm{~nm}$, density: $8.9 \mathrm{~g} / \mathrm{cm}^{3}$ ). In all cases, diameters near or below $100 \mathrm{~nm}$ were selected to ensure complete penetration of both $\mathrm{Xe}^{+}$and $\mathrm{He}^{+}$ions. 
The 50-nm silica sol was used "as-is". For materials supplied as dry powders, colloidal sols were prepared by slurrying approximately $0.10 \mathrm{~g}$ of the dry powder in $15 \mathrm{~mL}$ of 200 -proof ethanol. The samples were bath sonicated for 15 minutes in an attempt to disperse and breakup primary particle aggregates. Sub-monolayer coatings of particles were placed on copper TEM grids with a continuous carbon support film [purchased from Electron Microscopy Sciences (Hatfield, PA 19440)] by briefly dipping the grids into the prepared sols. The wetted grids were removed from the sol and allowed to dry evaporatively. To verify the coatings, the grids were imaged using the High Resolution TEM located at the William R. Wiley Environmental Molecular Sciences Laboratory, a national scientific user facility sponsored by the Department of Energy's Office of Biological and Environmental Research and located at Pacific Northwest National Laboratory (Richland, WA 99352). Sample electron micrographs for the 100-nm silica and 40-nm bismuth oxide are shown in Figs. 6.2 and 6.3

Ion irradiation studies were conducted using the IVEM-Tandem facility at Argonne National Laboratory (Argonne, Il 60439). IVEM-Tandem consists of a TEM connected to an ion accelerator, allowing samples to be imaged during irradiation. As such, any anisotropic deformation experienced by the colloidal particles can be imaged in real-time. The coated TEM grids are placed on a sample stage tilted $-15^{\circ}$ with respect to the to the viewing plane and electron beam to avoid shadowing. The ion beam is angled $-30^{\circ}$ with respect to the electron beam such that it strikes the sample at an angle of $-15^{\circ}$. An illustration of the tilt stage setup is shown in Fig. 6.4. The difference between the viewing and irradiation angle should allow direct observation of significant anisotropic deformation.

To minimize sample damage caused by the TEM, a $150 \mathrm{keV}$ electron beam was used. Prior to irradiation, the 50-nm silica sample was imaged for one hour to verify that no significant structure change or sample damage occurred as a result of imaging with the electron beam.

All samples were irradiated with $1 \mathrm{MeV}$ singly-charged Xe ions to verify that anisotropic deformation processes can influence the shape of these materials. Fluences as high as $8 \times 10^{15}$ ions $/ \mathrm{cm}^{2}$

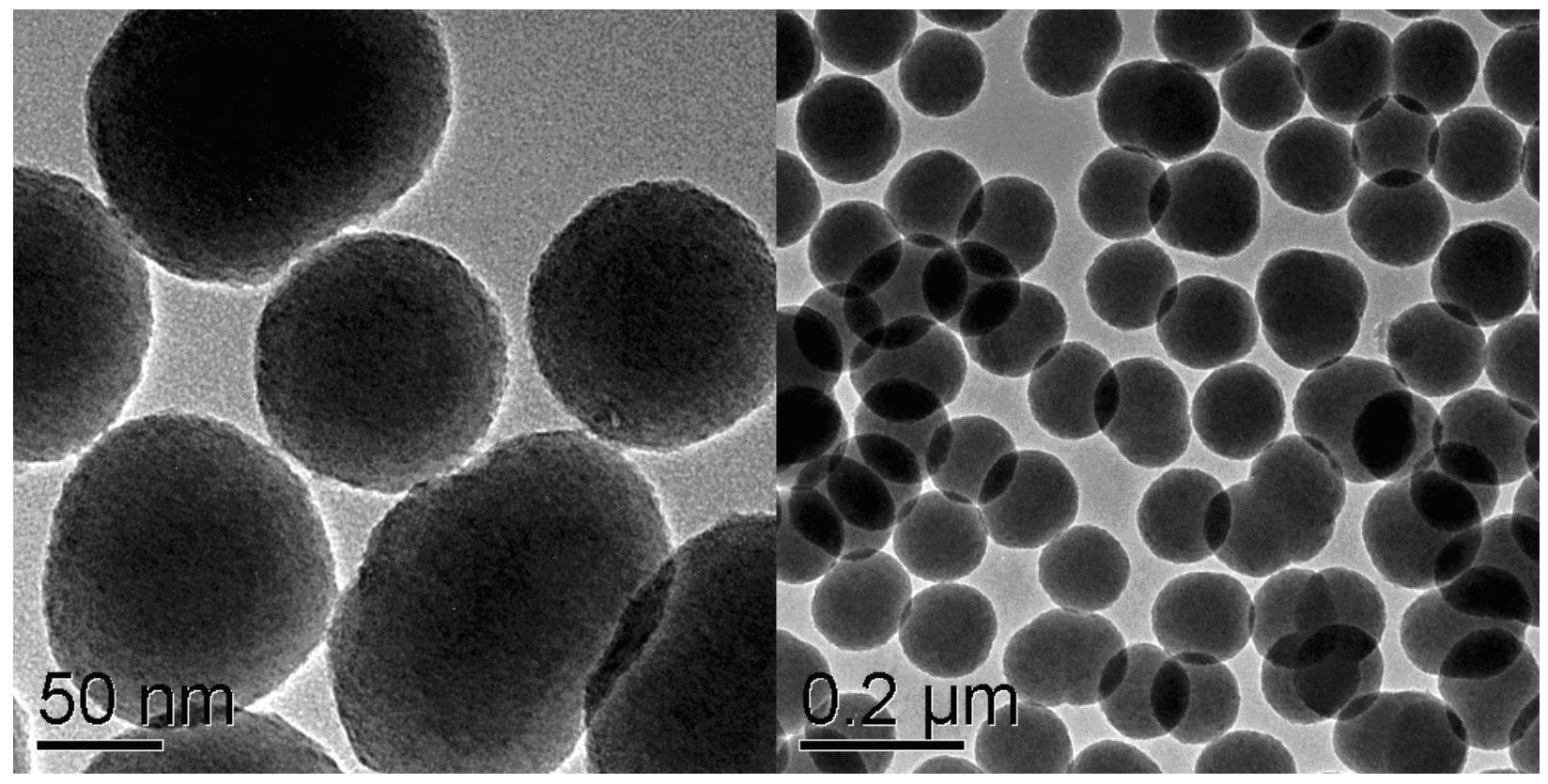

Figure 6.2. TEM of $100-\mathrm{nm}$ colloidal silica $\left(\mathrm{SiO}_{2}\right)$. There is little obvious fusing between adjacent particles. Primary particles are nearly spherical and are relatively monodisperse. 


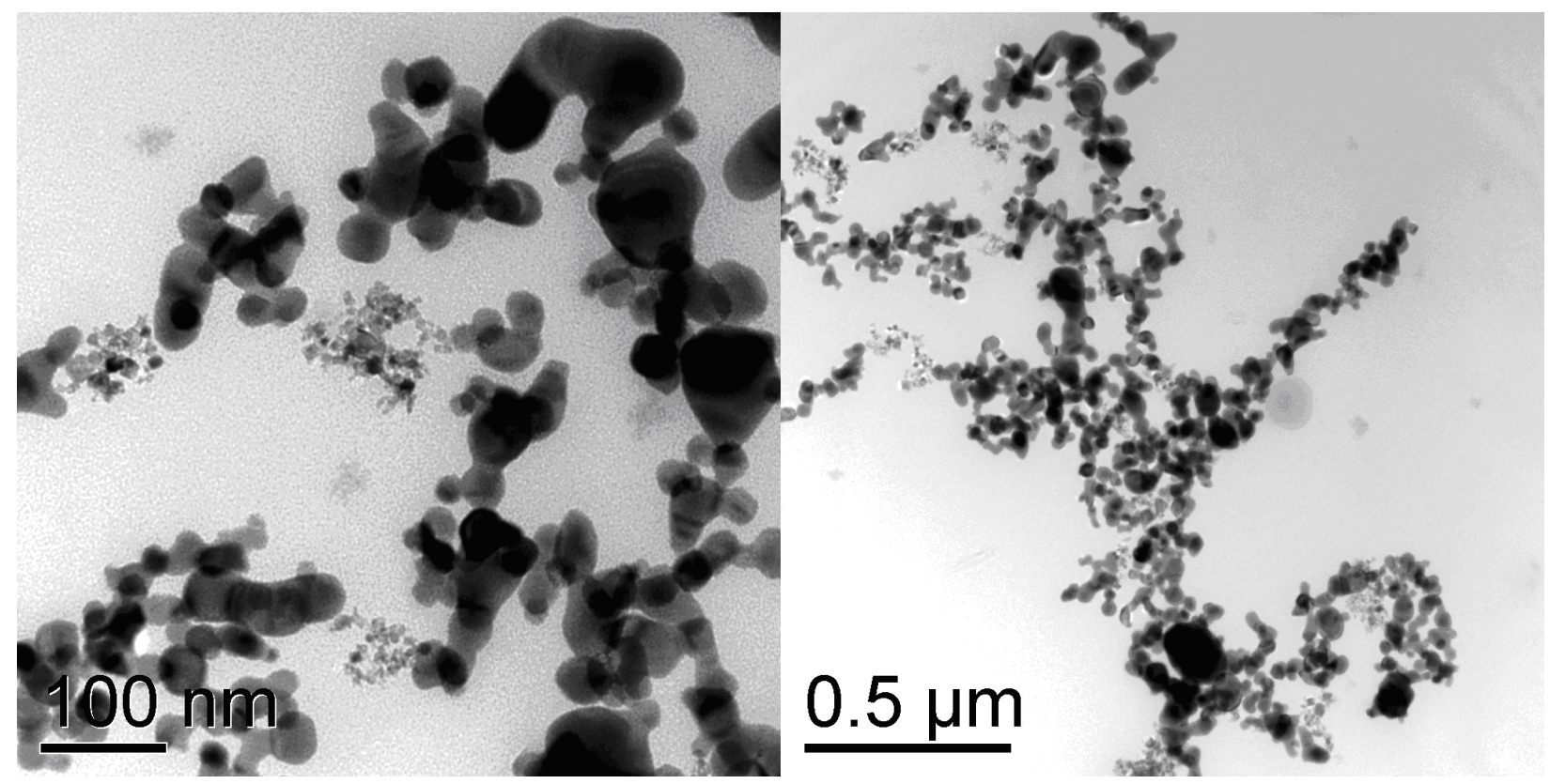

Figure 6.3. TEM of 40-nm polydisperse colloidal bismuth oxide $\left(\mathrm{Bi}_{2} \mathrm{O}_{3}\right)$. but aggregated. Analysis of the micrograph suggests that the bismuth oxide is crystalline.

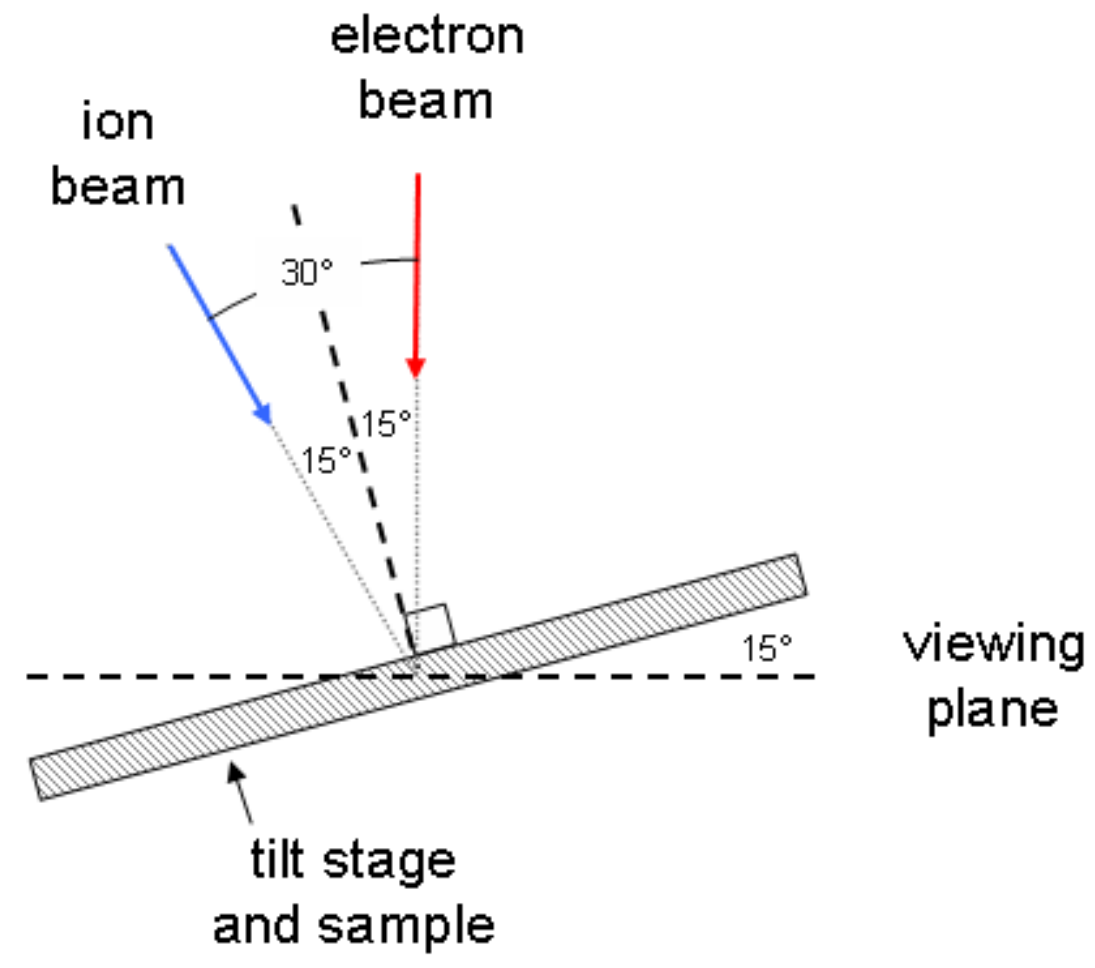

Figure 6.4. Sample stage setup for ion irradiation experiments. Stage is tilted $-15^{\circ}$ with respect to the electron beam. Ion beam is strikes the sample at a $15^{\circ}$ angle 
and $1 \times 10^{15}$ ions $/ \mathrm{cm}^{2}$ were tested for silica and bismuth oxide colloids, respectively. The influence of $500 \mathrm{keV}$ singly-charged He ions on the 50-nm silica samples was examined to simulate colloid damage / deformation resulting from $\alpha$ radiation. Helium ion fluences as high as $2 \times 10^{15}$ ions $/ \mathrm{cm}^{2}$ were tested. The range of fluences employed should be representative of the cumulative fluence in Hanford TRU waste streams generated from 1940-1950. All experiments were run at $30^{\circ} \mathrm{C}$.

\subsection{Results and Discussion}

Figure 6.5 presents images of 50-nm silica particles prior to and after irradiation with $1 \mathrm{MeV} \mathrm{Xe}^{+}$ ions. The total fluence applied to the sample between the presented images is $1.2 \times 10^{15}$ ions $/ \mathrm{cm}^{2}$. For the entire run, no significant oblation of the particle is observed. Although anisotropic deformation does not appear to occur over these fluences, there are some minor changes in the particle shape. Specifically, the particle perimeters appear to become more ragged over fluences from 0 to $2 \times 10^{14}$ ions $/ \mathrm{cm}^{2}$. The formation of spoke-like structures around the particles is observed (see Fig. 6.6 and suggests the ion beam sputters silica from the primary particle to the carbon film beneath. From $2 \times 10^{14}$ ions $/ \mathrm{cm}^{2}$ to $5.6 \times 10^{14}$ ions $/ \mathrm{cm}^{2}$, the sputtered spokes become slightly more visible and elongated but do not change significantly upon continued irradiation. Overall, no obvious change in particle shape of the $50-\mathrm{nm}$ silica is seen over the duration of experiment. Two possibilities exist: 1) the experimental fluences examined were not sufficient to deform or 2) surface forces are significant enough to retard the internal deformation processes. The former implies the small size of the colloid may not present enough material to stop or interact with a significant number of ions. The total energy input into the colloid is lower than expected, and requires much higher fluences (potentially an order of magnitude higher) to achieve the desired deformation. Because of time constraint at the IVEM-TANDEM facility, fluences up to and exceeding $1 \times 10^{16}$ ions $/ \mathrm{cm}^{2}$ could not be tested. The second possibility implies that curvature forces correct an isotropic deformation brought about by localized thermal spikes. At length scales below $100 \mathrm{~nm}$, solid diffusion can correct any localized increases in particle curvature. This phenomenon is the well-known cause of solid sintering in nanoscopic colloidal particles in contact with one another [see (Zachariah and Carrier 1999)]. It is possible that the localized curvature increase that occurs particle perimeter on the plane perpendicular to the ion beam is corrected by diffusion of solid to the short-axis.

Figure 6.7 presents images of a selected 100 -nm silica particle prior to and after irradiation with $1 \mathrm{MeV} \mathrm{Xe} e^{+}$ions. The total fluence applied to the particle between the presented images
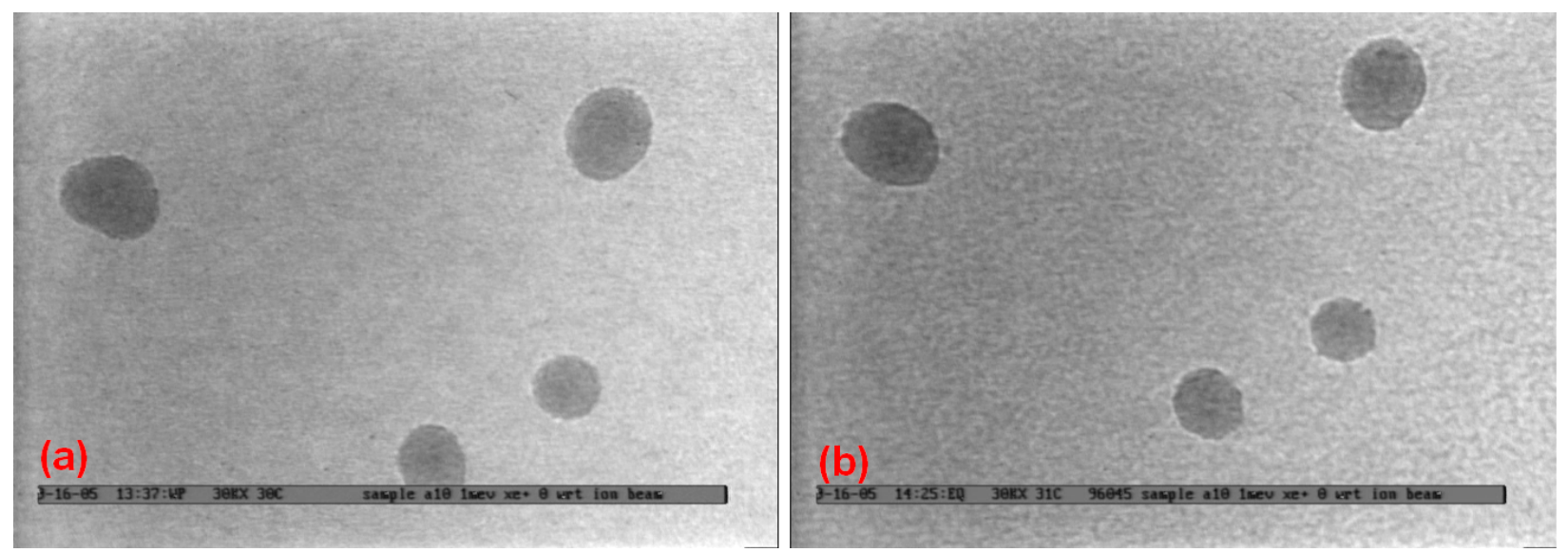

Figure 6.5. 50-nm spherical silica $\left(\mathrm{SiO}_{2}\right)$ (a) before irradiation and (b) after irradiation (fluence: $1.2 \times 10^{15}$ ions $/ \mathrm{cm}^{2}$ ) with $1 \mathrm{MeV} \mathrm{Xe}{ }^{+}$ions 


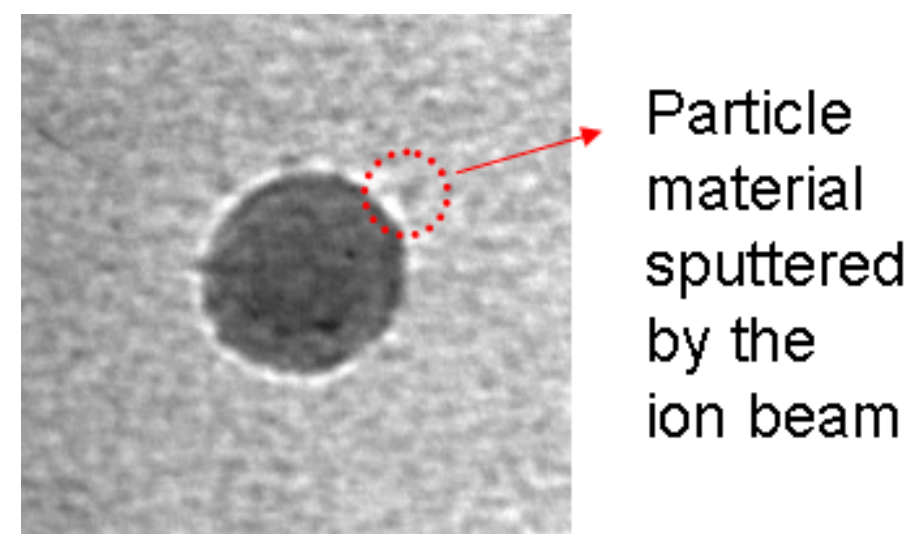

Figure 6.6. Evidence of sputtering during irradiation of 50-nm spherical silica $\left(\mathrm{SiO}_{2}\right)$ with $1 \mathrm{MeV} \mathrm{Xe}$ ions

is $8.0 \times 10^{14}$ ions $/ \mathrm{cm}^{2}$. Direct comparison of before and after images shows some oblation of the particle. The change is not drastic, but is sufficient to confirm the process of anisotropic deformation in 100-nm colloidal silica. Because of sample motion that resulted from the ion beam striking the sample and thermal expansion of the TEM grid, it was difficult to track the rate of deformation. The process of shape change was relatively slow, as no abrupt distortion of the particle shape or sputtering of material was observed.

Figure 6.8 presents images of a bismuth oxide cluster prior to and after irradiation with $1 \mathrm{MeV}$ $\mathrm{Xe}^{+}$ions. The total fluence applied between the presented images is $2.0 \times 10^{14}$ ions $/ \mathrm{cm}^{2}$. In contrast to the silica samples, the change in bismuth oxide upon application of the ion beam is abrupt and noticeable. There is significant growth of the particle in the viewing plane and ion damage effect (i.e., cluster bubbling or the generation of new nodes) are visible in real time. Examination of the crystal structure with XRD suggest that the bismuth oxide nanoparticles undergo a crystalline to amorphous transition upon the application of $1 \mathrm{MeV} \mathrm{Xe}$ ions

Figure 6.9 presents images of 50-nm silica particles prior to and after irradiation with $500 \mathrm{keV}$ $\mathrm{He}^{+}$ions. The total fluence applied between the presented images is $1.0 \times 10^{15}$ ions $/ \mathrm{cm}^{2}$. The particles in this image do not appear to deform over the course of the experiment. While the perimeters of the particle tend to become more smooth, no significant change in shape or silica sputtering is observed. Except for the absence of material transfer to the carbon film, these results are similar to the $50 \mathrm{~nm}$ silica tests with Xe ions.

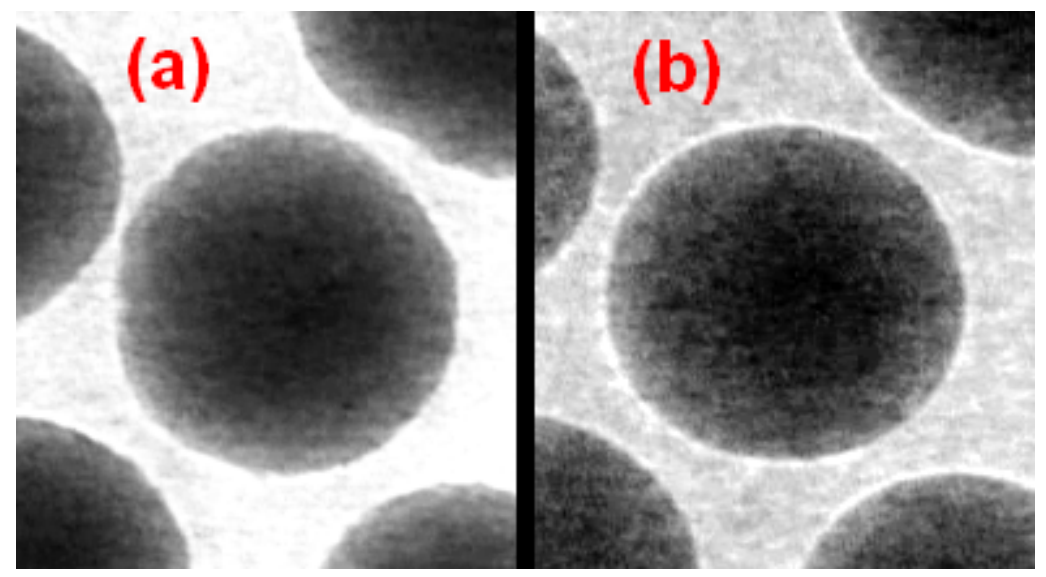

Figure 6.7. 100-nm spherical silica $\left(\mathrm{SiO}_{2}\right)$ (a) before irradiation and (b) after irradiation (fluence: $8.0 \times 10^{14}$ ions $/ \mathrm{cm}^{2}$ ) with $1 \mathrm{MeV} \mathrm{Xe}^{+}$ions 

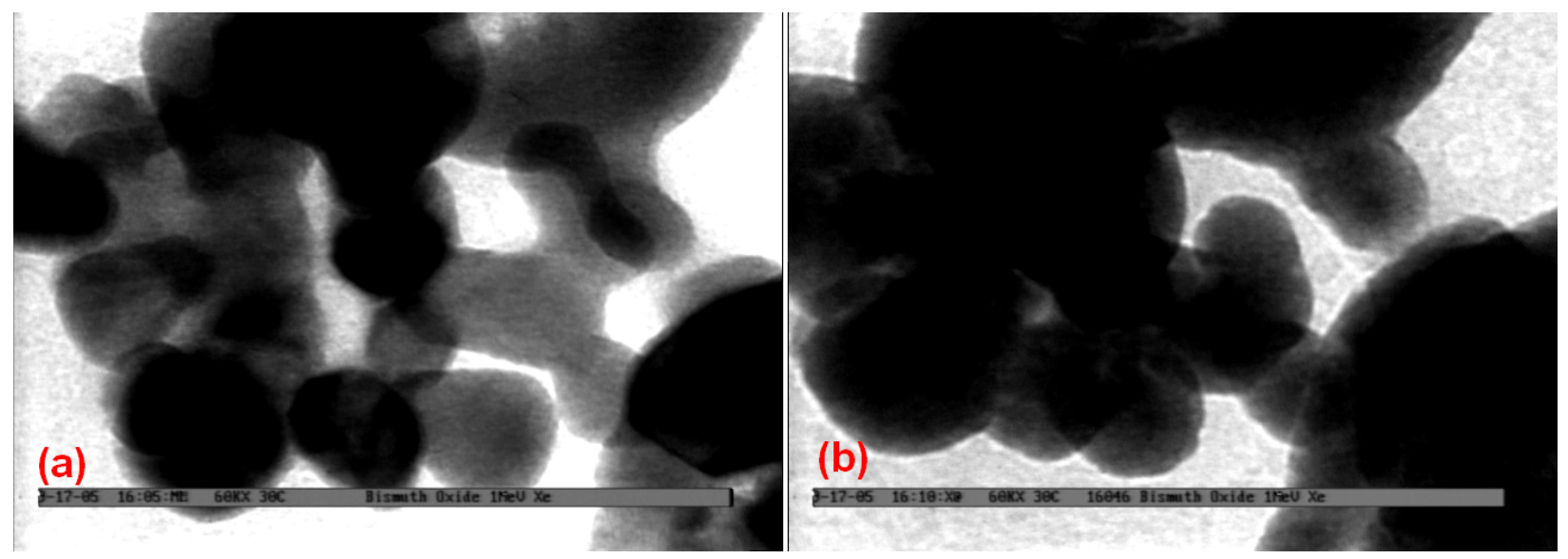

Figure 6.8. 40-nm spherical bismuth oxide $\left(\mathrm{Bi}_{2} \mathrm{O}_{3}\right)$ (a) before irradiation and (b) after irradiation (fluence: $2.0 \times 10^{14}$ ions $/ \mathrm{cm}^{2}$ ) with $1 \mathrm{MeV} \mathrm{Xe}^{+}$ions
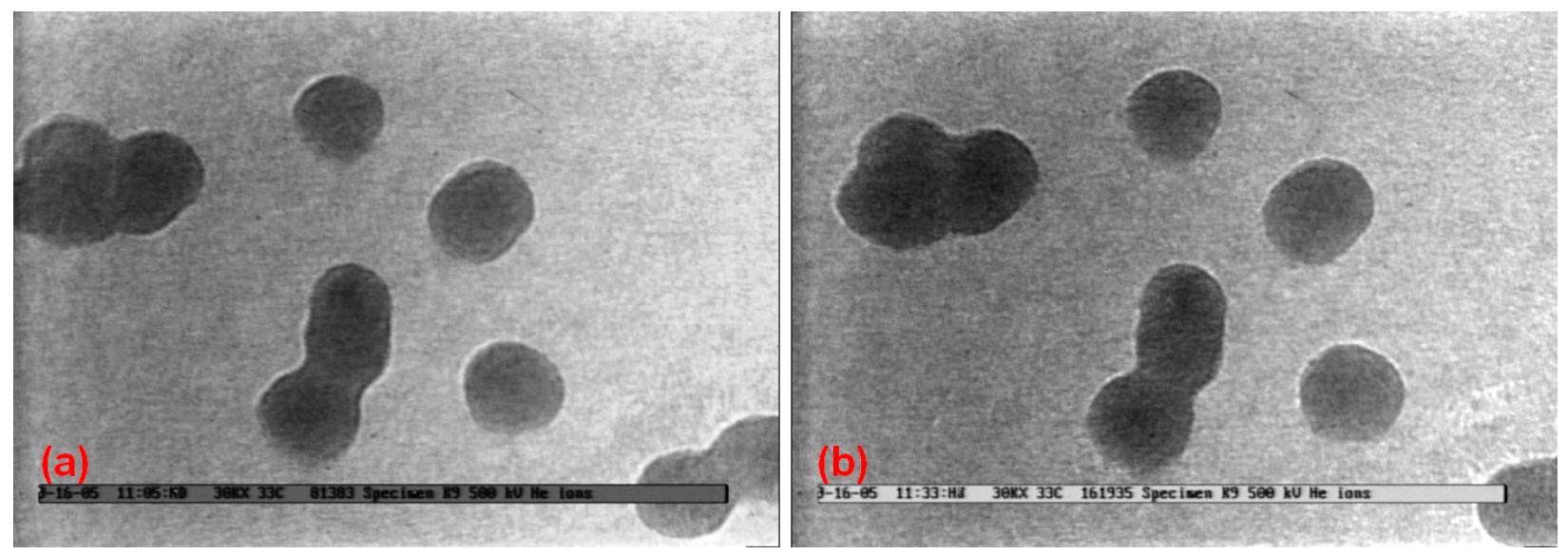

Figure 6.9. 50-nm spherical silica $\left(\mathrm{SiO}_{2}\right)$ (a) before irradiation and (b) after irradiation (fluence: $1.0 \times 10^{15}$ ions $/ \mathrm{cm}^{2}$ ) with $500 \mathrm{keV} \mathrm{He}^{+}$ions

\subsection{Conclusions}

The goal of the study presented herein was to determine if anisotropic deformation affected select mineral solids found in Hanford TRU sludges. The driving factor is the anisotropic particle shapes found in actual Hanford tank sludges (see Chapter 2). Irradiation of colloidal silica and bismuth oxide produced mixed results. For silica irradiated with Xe, noticeable anisotropic deformation was observed for $100 \mathrm{~nm}$ spherical particles at a fluence of $8.0 \times 10^{14} \mathrm{ions} / \mathrm{cm}^{2}$. Smaller $50 \mathrm{~nm}$ silica particles were not anisotropically deformed by either Xe and He irradiations. It is possible that either the experimental fluences examined (up to $1.25 \times 10^{15}$ ions $/ \mathrm{cm}^{2}$ ) were not sufficient to effect deformation or surface forces are significant enough to retard the internal deformation processes. Ion irradiation of bismuth oxide effected significant expansion of the sample in the viewing plane and caused a crystalline to amorphous transition. Time constraints prevented the study of higher fluences and the effects of He ion irradiation on $100 \mathrm{~nm}$ silica and bismuth. As such, it is unclear if anisotropic deformation can be accomplished by $\alpha$ radiation. The experimental results indicate that deformation of materials present in Hanford TRU sludges can occur as a result of heavy-ion bombardment. Examination of different particle sizes suggests that smaller particles could be resistant to anisotropic deformation. Whether $\alpha$ can effect changes in particle-shape and morphology remains an open question. The issue could be addressed by irradiating larger colloids (i.e., particles $100-500 \mathrm{~nm}$ in diameter) with He ions. 



\subsection{Methodology for Colloidal Aggregation Modeling}

The shear thinning behavior of colloidal suspensions is based on the formation and growth of colloidal aggregates. The key to modeling suspension behavior is to accurately predict the aggregate size distribution that, in turn, determines the suspension properties such as effective conductivity and effective viscosity. The aggregate size distribution is a function of the local flow conditions, so there is coupling in both directions.

The aggregate size distribution is determined by solving the population balance equation (PBE) at every computational node in the domain of interest. The Direct Quadrature Method of Moments (DQMOM), which represents the evolution of the aggregate size distribution using a small number of scalar values, is described in Section 7.1. The lattice kinetics simulation method, described in Section 7.2, is used to model the system flow and diffusion transport of the scalar quantities. The original version of DQMOM assumes that the orthokinetic aggregation rate is based solely on the collision rate of the particles and aggregates. However, suspensions where the particle interactions have an energy barrier require a sufficient shear energy to achieve aggregation. A description of this shear-activated aggregation is provided in Section 7.3. This modeling approach is being validated using data obtained from the University of Washington. The progress to date is described in Section 7.4.

\subsection{Description of the Direct Quadrature Method of Moments}

The simulation approach we are using to simulate colloid agglomerate growth is the DQMOM method (Marchisio and Fox 2005). The primary advantage of this method is that the particle size distribution may be described with reasonable accuracy using a limited (typically six) number of transportable scalars.

The DQMOM method begins with the population balance equation

$$
\frac{\partial n(L ; x, t)}{\partial t}+\frac{\partial}{\partial x_{i}} U_{i} n(L ; x, t)-\frac{\partial}{\partial x_{i}}\left[\bar{D} \frac{\partial n(L ; x, t)}{\partial x_{i}}\right]=S(L ; x, t)
$$

where $n$ is the particle size distribution, $L$ is the internal coordinate vector, $\bar{D}$ is the diffusion coefficient and $S$ is the source term, which contains molecular growth, aggregation and breakage. The PSD is expressed using a quadrature approximation of order $N$

$$
n(L ; x, t)=\sum_{\alpha=1}^{N} w_{\alpha}(x, t) \delta\left[L-L_{\alpha}(x, t)\right]
$$

where $\delta$ is the Dirac delta function, $w_{\alpha}$ are the weights and $L_{\alpha}$ are the 'abscissas' of the approximation.

Substituting into the PBE, each of the weights and abscissas develops a corresponding transport equation with source terms. The weight and abscissa equation source terms are calculated from 
the moment source terms, given by the expression

$$
\bar{S}_{k}=\frac{1}{2} \sum_{i=1}^{N} \omega_{i} \sum_{j=1}^{N} \omega_{j}\left(L_{i}^{3}+L_{j}^{3}\right)^{\frac{k}{3}} \beta_{i j}-\sum_{i=1}^{N} L_{i}^{k} \omega_{i} \sum_{j=1}^{N} \beta_{i j} \omega_{j}+\sum_{i=1}^{N} a_{i} \bar{b}_{i}^{(k)} \omega_{i}-\sum_{i=1}^{N} L_{i}^{k} a_{i} \omega_{i}
$$

where $\beta_{i j}$ are the aggregation kernels, the probability that aggregates of different sizes will combine, $a_{i}$ are the breakage kernels, the probability that an aggregate of a certain size will break into two fragments, and $b_{i}$ are the fragmentation kernels, the probability that certain size fragments will be formed. Each of these kernels is a function of local flow conditions.

The abscissa $L$ corresponds to the net solid volume contained in the aggregate. However, the collision length, $L_{c}$, will be significantly larger since colloidal aggregates are fractal objects, that is, porous particle networks that decrease in density as a function of distance from the center of the aggregate. This behavior is characterized by the fractal dimension, $D_{f}$, which is used to calculate the collision size using

$$
L_{c}=L\left(\frac{L}{d_{0}}\right)^{\frac{3}{D_{f}}}
$$

The connection of two aggregates to form a larger aggregate is assumed to occur by either of two different mechanisms. Orthokinetic or "shear induced" aggregation occurs when a shearing flow field brings two aggregates into contact. The aggregation kernel for this is

$$
\beta_{i, j}^{s h}=\frac{4}{3} F_{i j} G\left(L_{c, i}+L_{c, j}\right)^{3}
$$

where $G$ is the local shear rate and $F$ is the probability of aggregation for each collision. For the original DQMOM model, the probability $F$ was assumed to equal one. However, for colloidal systems where the particle-particle interactions have an energy barrier, the function $F$ takes a more complicated form. This will be discussed in more detail in Section 7.3.

The kernel for Brownian aggregation of fractal aggregates is

$$
\beta_{i, j}^{B r}=\frac{2 k T}{3 \mu_{0}}\left(\frac{1}{L_{c, i}}+\frac{1}{L_{c, j}}\right)\left(L_{c, i}+L_{c, j}\right)
$$

The breakage kernel is assumed to have the form

$$
a_{i}=A G^{\gamma} L_{i}^{\frac{1}{3}}
$$


where $\gamma$ is in the range of 1.6. The fragmentation kernal selected is for symmetric fragmentation and has the form

$$
b_{i}^{(k)}=2^{\frac{(3-k)}{3}} L_{i}^{k}
$$

The goal of this task is to predict the agglomerate size distribution as a function of location based on operating conditions. The DQMOM method has been implemented into the lattice kinetics computer program and tested for colloidal systems.

\subsection{Description of the Lattice Kinetics Method}

The lattice kinetics flow solution method is similar to the lattice Boltzmann method (Sukop and Thorne 2005) in many respects. The single particle distribution function $f(\mathbf{x}, \mathbf{v}, t)$ is the probability of finding particles at position $\mathbf{x}$ with velocity $\mathbf{v}$ at time $t$. The Boltzmann equation describes the evolution of the distribution function

$$
\frac{\partial f}{\partial t}+v \cdot \nabla f=-\frac{f-f^{e q}}{\tau}
$$

where $\tau$ is the relaxation time and $f^{e q}$ is the equilibrium Maxwell distribution. To solve this equation on a computer, the spatial domain is discretized into a finite number of lattice sites, where each lattice site has values for density, pressure, flow, etc. The single-particle distribution function, which describes the probability of a particle traveling along a particular direction and speed, is discretized to form a finite set of displacement vectors connecting each lattice site to adjacent sites.

The lattice Boltzmann equation describes the evolution of the discretized particle distribution function, $f_{i}(\mathbf{x}, t)$, along direction $i$ as a function of time. The new time distribution function is given by the equation

$$
f_{i}\left(x+\Delta t e_{i}, t+\Delta t\right)-f_{i}(x, t)=-\frac{1}{\tau}\left[f_{i}(x, t)-f_{i}^{e q}(x, t)\right]
$$

where $\tau$ is a linear relaxation parameter and $f^{e q}$ is the local equilibrium distribution. The direction vectors for two dimensions are

$$
\begin{aligned}
e_{0} & =0 \\
e_{1-4} & =\left\{\cos \left[\frac{\pi}{2}(i-1)\right], \sin \left[\frac{\pi}{2}(i-1)\right]\right\} c \\
e_{5-8} & =\sqrt{2}\left\{\cos \left[\frac{\pi}{2}(i-5)+\frac{\pi}{4}\right], \sin \left[\frac{\pi}{2}(i-5)+\frac{\pi}{4}\right]\right\} c
\end{aligned}
$$


The local equilibrium is expressed in the form of a quadratic expansion of the Maxwellian distribution

$$
f_{i}^{e q}=w_{i} \rho\left[1+\frac{3 e_{i} \cdot u}{c^{2}}+\frac{9\left(e_{i} \cdot u\right)^{2}}{2 c^{4}}-\frac{3 u^{2}}{2 c^{2}}\right]
$$

where $c$ is the reference lattice speed, $c=\Delta x / \Delta t$ and the weight coefficients, $w_{i}$ for a threedimensional system are $w_{0}=1 / 3, w_{1-6}=1 / 18$ and $w_{7-18}=1 / 36$.

The lattice Boltzmann equation may be rewritten to take the form

$$
f_{i}\left(x+\Delta t e_{i}, t+\Delta t\right)=f_{i}^{e q}(x, t)+\left(1-\frac{1}{\tau}\right)\left[f_{i}(x, t)-f_{i}^{e q}(x, t)\right]
$$

The first term on the right hand side represents the distribution function for the special case of $\tau=1$, which corresponds to the kinetic viscosity

$$
v=\frac{1}{6}\left(\tau-\frac{1}{2}\right) \frac{\Delta x^{2}}{\Delta t}
$$

The second term is the additional shear term resulting from a kinematic viscosity that is different from the reference case. The lattice kinetics method replaces this second term with an explicit calculation of the local stress based on velocity gradients

$$
f_{i}\left(x+\Delta t e_{i}, t+\Delta t\right)=f_{i}^{e q}(x, t)
$$

where

$$
f_{i}^{e q}=w_{i} \rho\left[1+\frac{3 e_{i} \cdot u}{c^{2}}+\frac{9\left(e_{i} \cdot u\right)^{2}}{2 c^{4}}-\frac{3 u^{2}}{2 c^{2}}+A \Delta t\left(\frac{\partial u_{\alpha}}{\partial x_{\beta}}+\frac{\partial u_{\beta}}{\partial x_{\alpha}}\right) e_{i \alpha} e_{i \beta}\right]
$$

and the coefficient $\mathrm{A}$ is related to the kinematic viscosity by the expression

$$
A=0.75-4.5 \vee \frac{\Delta t}{\Delta x^{2}}
$$


The new time density and velocity values are determined using the expressions

$$
\begin{gathered}
\rho\left(x+\Delta t e_{i}, t+\Delta t\right)=\sum_{i} f_{i}^{e q}(x, t) \\
\rho u\left(x+\Delta t e_{i}, t+\Delta t\right)=\sum_{i} f_{i}^{e q}(x, t) e_{i}
\end{gathered}
$$

This procedure is repeated for the specified number of time steps until a steady-state solution is obtained. The result is a second-order solution to the Navier-Stokes and continuity equations.

\subsection{Adaptation of the DQMOM Method to Shear-Activated Aggregation}

The aggregation kernel used in the original DQMOM method assumes that the aggregation rate depends solely on the collision rate between aggregates of various sizes. However, some colloidal systems have particle-particle interactions with an energy barrier, which reduces the probability of aggregation for each collision, $F_{i j}$.

The potential interaction between two particles may be described using DLVO theory, which states that the total potential energy is the sum of a van der Waals (or dispersion) attractive term and a repulsive electrostatic double layer term

$$
V_{T}=V_{A}+V_{R}
$$

The van der Waals attraction is given by the expression

$$
V_{A}(D)=-\frac{A}{12 \pi D^{2}}
$$

where $D$ is the particle-particle separation and $A$ is the Hamaker constant, which is a function of the particle materials. The screened Coulomb repulsion term for low surface potentials is given by

$$
V_{R}(D)=2 \pi \varepsilon_{0} \varepsilon r \xi^{2} \exp (-\kappa D)
$$

where $r$ is the particle radius, $\varepsilon$ is the solvent permittivity, $\xi$ is the zeta potential and $\kappa$ is the Debye length. The location of the potential energy barrier, $V_{\max }$, is determined using the first derivative with respect to separation

$$
V_{\text {max }}=V_{T}\left(\frac{d V_{T}(D)}{d D}=0\right)
$$


The existence of a potential energy barrier results in a reduction or elimination of aggregation unless there is a mechanism for overcoming the barrier. Guery et al. (2006) reports the shearactivated aggregation of otherwise stable suspensions. The shearing motion creates hydrodynamic energy, which can be characterized by

$$
E_{\text {hyd }}=\eta G r^{2} \delta
$$

where $\eta$ is the viscosity, $G$ is the shear rate, $r$ is the radius and is the microscopic length over which the interparticle repulsive potential is effective. The probability for aggregation per collision takes the form of a thermally activated Arrhenius process

$$
F=\min \left[1, \exp \left(\frac{E_{h y d}-V_{\max }}{k_{B} T}\right)\right]
$$

Note that when the hydrodynamic energy exceeds the maximum energy barrier, the aggregation probability is one.

\subsection{Validation of Modeling Methodology}

It is hoped that this simulation methodology accurately predicts aggregate size distributions and ultimately shear stress-strain rate relationships for steady-state suspensions in a shear field given the system's colloid properties and shear rate. The accuracy of the predictions made by this simulation had not been tested against real systems. The objective is therefore to validate using an experimental data set provided by the John Berg research group at the University of Washington.

The data set provided to PNNL consisted of aggregate particle size distributions as a function of colloidal interaction parameters and applied shear rate. Systems of aqueous titania suspensions were aggregated at a controlled shear rate by adding known amounts of salt. These systems were convenient since, under the conditions used, particle interaction potentials can be calculated according to DLVO theory. Particle interactions as calculated using DLVO theory require the knowledge of certain colloidal parameters including the zeta potential. This quantity was measured using a Brookhaven Zeta-PALS.

Various controlled shear rates were obtained using a Physica MCR-300 rheometer. Stable titania suspensions at $\mathrm{pH} 10$ were sheared at controlled rates with the rheometer. Then salt solution was injected into the sample by syringe so that the aggregation process occurred within the shear field. Various amounts of salt were used in order to obtain particle interaction potentials ranging from repulsive to strongly attractive. The aggregation process was allowed to reach steady-state. It was assumed that a steady-state was achieved when the recorded shear stress at the given shear rate became constant with time.

Samples were then taken for particle size determination. These samples were immediately diluted in order to prevent further aggregation. Particle size distributions were determined by a settling technique which uses light obscuration to track sedimentation rate then calculates size based on Stokes Law. The equipment used for this measurement was a Horiba CAPA-500. 
A matrix of particle size distributions of systems with various zeta potentials aggregated at different shear rates with two different solids contents was collected using these measurement techniques.

A series of DQMOM/lattice kinetics simulations are being performed for the different solids loadings, solution chemistry (including $\mathrm{pH}$, zeta potential and salt concentration) and shear rate conditions. Transient simulations are run until the aggregate size distribution reaches steady state.

The results obtained from the DQMOM simulation are expressed in terms of pairs of weights and abscissas which contain information about the first six moments of the aggregate size distribution. To compare these results to the detailed distributions provided by the University of Washington, we must be able to convert these results to a similar form. The procedure for doing this is to define a series of cubic splines (Press et al. 1992) so that the moments match those of the DQMOM results. The tie points for the spline connections are adjusted and a merit function is constructed based on the difference between the moments of the test distribution and the DQMOM moments.

The results for the validation simulations have not been completed at the time of this report. A joint publication for submission to the journal Colloids and Surfaces A is in preparation. 



\subsection{Personnel Supported}

\subsection{Project Support At PNNL}

PNNL scientists and student interns supported by EMSP Project 90162 include:

- Dr. Adam P. Poloski, Principle Investigator

- Dr. Paul R. Bredt, Co-PI

- Dr. David R. Rector, collaborator

- Dr. Richard C. Daniel, collaborator

- Dr. Joel M. Tingey, collaborator

- Dr. Edgar C. Buck, collaborator

\subsection{University Collaborations}

University collaborations supported by EMSP Project 90162 include:

- Dr. John C. Berg, Co-PI, Professor of Chemical Engineering, University of Washington

- Dr. A. Eduardo Sáez, collaborator, Professor of Chemical and Environmental Engineering, University of Arizona

- Alvin Y. Huang, Graduate research assistant, University of Washington

- Peter B. Laxton, Graduate research assistant, University of Washington 



\subsection{Publications}

Support has allowed preparation of four manuscripts which have been or are in the process of being submitted for publication in peer reviewed journals:

- Poloski AP, PR Bredt, RC Daniel, and AE Sáez, "The contribution of frictional contacts to the shear strength of coarse glass bead powders and slurries", Rheologica Acta, published on the web, DOI: 10.1007/s00397-006-0105-3 (2006).

- Sáez AE, AP Poloski, and RC Daniel, "A Continuum Constitutive Model for Cohesionless Granular Flows", Submitted to Chem. Eng. Sci. (2006).

- Daniel RC, AP Polski, and AE Sáez, "Vane Rheology of Cohesionless Glass Beads", Manuscript in preparation.

- Rector DR, PB Laxton, and JC Berg, on the comparison of Lattice-Boltzmann simulations to sheared particle aggregate size distribution data for submission to Colloids and Surfaces A.

University collaboration has generated the following manuscripts which have been or are in the process of being submitted for publication in peer reviewed journals:

- Laxton PB and JC Berg, “Gel Trapping of Dense Colloids,” J. Coll. Interface Sci. 285 (2005).

- Huang AY and JC Berg, "High-salt stabilization of Laponite clay particles", J. Colloid Interface Sci. 296 (2006) 159-164.

- Laxton PB and JC Berg, "Relating clay yield stress to colloidal parameters", J. Colloid Interface Sci. 296 (2006) 749-755.

- Laxton PB and JC Berg, "Investigation of the link between micromechanical interparticle bond rigidity measurements and macroscopic shear moduli of colloidal gels", Colloids and Surfaces A, submitted September 1, 2006. 



\subsection{Project Accomplishments}

The overall objective of this project was to develop a fundamental understanding of how macroscopic TRU sludge rheological behavior correlates with particle-scale interactions and phenomena. To this end, extensive study of the rheology of dense slurries and colloidal suspensions in a variety of aqueous media has been conducted. With regard to coarse granular matter, models have been derived to allow characterization of the shear/mobilization strength and dynamic stress and velocity fields of powders and slurries using simple rheometric equipment, namely the vane rheometer. These models incorporate the effects of interparticle friction and cohesion, account for some properties of the suspending phase, and allow inclusion of process geometry effects such as friction between individual particles and confining walls. The development of granular theories surrounding the vane rheometer represents a significant step forward, as current techniques for characterizing dense sludge and slurry rheology require atypical test geometries that are not standard on commercial rheometers or advanced imaging methods (such as magnetic resonance imaging $[\mathrm{MRI}])$. A continuum model has been developed to allow prediction of three-dimensional granular flows. Because the formulation of this model is independent geometry, it can be applied to study of pipeline flow and mechanical agitation of slurries. From these accomplishments, it is now feasible to characterize the frictional and cohesive behavior of coarse grained slurries using conventional rheometers. The results of these analyses can be applied to scaled-up operations through the use of the continuum model developed herein. Work on coarse granular rheology has resulted in three manuscripts for publication in peer reviewed journals (one in-press, one submitted for peer review, and another in preparation).

Study of fine colloidal materials similar to those present in Hanford tank waste indicate that particle shape deformation can occur as a result of heavy-ion bombardment. It is speculated that similar processes may occur in tank materials as a result of long-term irradiation with alphaparticles, resulting in changes in the overall rheology because of alteration of dispersed phase morphology. A simulation methodology has been developed to predict the colloidal aggregate size distribution based on local flow conditions. The simulation uses the lattice Boltzmann/ lattice kinetics method to obtain the system hydrodynamics. This method is an improvement on conventional computational fluid dynamics in that it allows for parallel processing, reducing run time. The direct quadrature method of moments (DQMOM) method is used to solve the aggregate population balance equation at every computational node based on local flow and chemistry conditions. The resulting aggregate size distributions can then be used to predict the shear stress-strain rate relationships, which feed back into the flow solution. The methodology is currently being validated using data obtained from particle size information obtained from the University of Washington.

Overall, the project was successful in providing a means by which select macroscopic properties of Hanford tank sludge can be characterized and related to particle-level interaction. 



\subsection{Future Work}

With regard to the studies presented in this report, future work should be aimed at refining the characterization techniques and modeling equations and testing their effectiveness for predicting velocity and stress fields in pilot-scale mixing vessels or pipeline flow. The model for granular system dynamics is dependent on functional forms for scaling of frictional and dilatancy effects with inertial number obtained from two-dimensional granular media simulations. While applied successfully to both granular mobilization and rheometric flows, there is still some uncertainty regarding the nature and influence of anisotropic stresses on the measured flow behavior, especially with regard to dynamic systems. In one example, this ambiguity is manifest by lower than expected quasi-static forces on a mixing impeller at low rotational rates. It is speculated that the impeller alters the granular stress field. To resolve this particular issue, direct measurements of normal stress fields should be attempted for granular systems under shear (using a load cell similar to the one used to measure the static stress fields herein). The challenge here is to design a sensor whose footprint is small enough not to alter the stress field it is measuring.

Application of the granular continuum models is only done on the bench-scale. There is some uncertainty as to their ability to correctly describe pilot- and engineering-scale systems. Mixing and flow testing of dense granular slurries in large tanks could be potentially carried out at the High Bay Testing Facility (336) Building at PNNL. These tests would focus on verifying the proper form of the granular normal stress distribution and on validating the shear strength and continuum models for pilot-scale granular stress and velocity fields.

With regard to the colloidal phenomena discussed in this report, studies of anisotropic deformation were inconclusive as to whether of $\alpha$ radiation could induce a shape change. However, the al pha radiation studies were conducted using particles that resisted deformation even under heavy-ion bombardment. This issue could be addressed by irradiating larger colloids (i.e., particles 100-500 nm in diameter) with He ions. Bismuth oxide would present the best candidate for these studies, as it was significantly altered by irradiation with heavy ions. Studies covering a broader range of particle sizes would also be useful, as they would further elucidate the role particle size and surface forces play in resisting anisotropic deformation. 



\subsection{References}

Ancey C, and P Coussot, "Transition from frictional to viscous regimes for granular suspensions," C.R. Acad. Sci. Paris sér. II. 327, 515 (1999).

Ancey C, P Coussot, and P Evesque, "A theoretical framework for granular suspensions in a steady simple shear flow", J. Rheol. 43, 1673-1699 (1999).

Ancey C, "Role of lubricated contacts in concentrated polydisperse suspensions", J. Rheol. 45, 1421 (2001).

Ancey C, and H Jorrot, "Yield stress for particle suspensions within a clay dispersion," J. Rheol. 45, 297 (2001).

Andres, UT, "Equilibrium and motion of a sphere in a viscoplastic liquid," Doklady Akademii Nauk SSSR, v 133, n 4, p 777-780 (1960).

Bamberger JA, PA Meyer, JR Bontha, CW Enderlin, AP Poloski, JA Fort, ST Yokuda, HD Smith, F Nigl, M Friedrich, DE Kurath, GL Smith, JM Bates, MA Gerber, and DA Wilson, "Technical Basis for Testing Scaled Pulse Jet Mixing Systems for Non-Newtonian Slurries,” PNWD-3551, Battelle-Pacific Northwest Division, Richland WA (2005).

Barnes HA, and NQ Dzuy, "Rotating vane rheometry - a review," J. Non-Newtonian Fluid Mech., 1-14 (2001).

Behringer RP, D Howell, L Kondic, S Tennakoon, and C Veje, "Predictability and granular materials," Physica D 133, 1-17 (1999).

Bertho Y, F Giorgiutti-Dauphine, and J Hulin, "Dynamical Janssen effect on granular packings with moving walls,' Phys. Rev. Lett. 90, (2003).

Benarie MM, "Rheology of Granular Material II - A Method for the Determination of Intergranular Cohesion", Brit. J. Appl. Phys. 12, 514 (1961).

Beris AN, JA Tsamopoulos, RC Armstrong, and RA Brown, “Creeping motion of a sphere through a Bingham plastic," J. Fluid Mech., v 158, p 219-244 (1985).

Campbell CS, “Rapid Granular Flows”, Ann. Rev. Fluid Mech., 22, 57-92 (1990).

Chang CY, and Smith PA, "Rheological characterization of nuclear waste slurries," Particulate Sci. Technol. 14(2), 165-180 (1996).

Cheng DCH and R Farley, "Comments on the Paper 'Rheological Behaviour of Powder in a Rotational Viscometer' by Hiroshi Kuno and Kozo Kurihara”, Rheologica Acta 5(1), 53-56 (1966).

Cheng X, JB Lechman, A Fernandez-Barbero, GS Grest, HM Jaeger, GS Karczmar, ME Möbius, and SM Nagel "Three-Dimensional Shear in Granular Flow," Phys. Rev. Lett. 96, 038001 (2006).

Dabak T, and O Yucel, "Modeling of the concentration and particle size distribution effects on the rheology of highly concentrated suspensions" Powder Tech. 52, 193-206 (1987).

da Cruz F, S Emam, M Prochnow, JN Roux, and F Chevior , "Rheophysics of Dense Granular Materials: Discrete Simulation of Plane Shear Flows”, Phys. Rev. E 72, 021309 (2005). 
de Gennes PG, “Granular Material: A Tentative View,” Rev. Modern Phys. 71, S374-S382 (1999).

Depken M, W van Saarloos, and M van Hecke, "Continuum approach to wide shear zones in quasistatic granular matter”, Phys. Rev. E 73, 031302 (2006).

Dzuy NQ, and DV Boger, "Direct yield stress measurement with the vane method," J. Rheology 29, 335-348 (1985).

Fayed ME, and L Otten (Eds), Handbook of Powder Science and Technology, Chapman and Hall (1997).

Gentils A, L Thome, J Jagielski, L Nowicki, S Klaumunzer, F Garrido, M Beauvy, "Damage production in cubic zirconia irradiated with swift heavy ions”, Nucl. Instrum. Meth. B 218, 457-460 (2004).

Goldhirsch I, “Rapid Granular Flows”, Ann. Rev. Fluid Mech., 35, 267 (2003).

Guery J, E Betrand, C Rouzeau, P Levitz, DA Weitz and J Bibette, "Irreversible Shear-Activated Aggregation I Non-Brownian Suspensions”, Phys. Rev. Let. 96, 198301 (2006)

Iverson RM and JW Vallance, "New Views of Granular Mass Flows", Geology 29(2), 115118 (2001).

Jagielski J, A Gentils, L Thome, L Nowicki, F Garrido, and S Klaumunzer, "Channeling study of the damage induced in zirconia irradiated with high-energy heavy ions", Nucl. Instrum. Meth. B 219, 626-630, (2004).

Janssen HA, "Versuche ber Getreidedruck in Silozellen," Zeitschr. d. Vereines deutscher Ingenieure, 39, 1045-1049 (1895).

Jenkins JT, and MW Richman, "Kinetic Theory for Plane Flows of a Dense Gas of Identical, Rough, Inelastic, Circular Disks", Phys. Fluids, 28, 3485-3494 (1985).

Jop P, Y Forterre, and O Pouliquen, Nature 441, 727 (2006).

Kuno H and K Kurihara, "Rheological Behaviour of Powder in a Rotational Viscometer", Rheologica Acta 4(1), 73-74 (1965).

Kuno H and M Senna, "Rheological Behavior of Powder in a Rotational Viscometer", Rheologica Acta 6(3), 284-288 (1967).

Landel R, BG Moser, and AJ Bauman, 4th International Congress on Rheology (1963) Proceedings Part 2, Interscience Publishers, NY, 663 (1965).

Laxton PB, and JC Berg, "Gel Trapping of Dense Colloids," J. Coll. Interface Sci. 285 (2005).

Lee WK, "The rheological nature of solid pressures of granular media," Powder Tech. 51, 261266 (1987).

Liddell PV, and DV Boger, "Yield stress measurement with the vane," J. Non-Newtonian Fluid Mech. 63, 235-261 (1996).

Marchisio D and RO Fox, "Solution of population balance equations using the direct quadrature method of moments," J. Aerosol Sci. 36, 43-73 (2005).

Meyer P, and B Wells, "Understanding Gas Release Events in Hanford Double Shell Tanks," WM'OO, Proceedings of the Symposium on Waste Management, Tucson, Arizona (2000). 
MiDi GDR, “On Dense Granular Flows”, Eur. Phys. J. E 14, 341-364 (2004).

Mueth DM, DF Debregeas, GS Karczmar, PJ Eng, SR Nagel, HM Jaeger, "Signatures of Granular Microstructure in Dense Shear Flows”, Nature 406, 385-389 (2000).

Mueth DM, "Measurements of Particle Dynamics in Slow, Dense Granular Couette flow”, Phys. Rev. E 67, 011304 (2003).

Nedderman RM, Statics and Kinematics of Granular Materials, Cambridge University Press, (1992).

Norato MA, TJ Steeper, DJ McCabe, "Demonstration of an Ion Exchange Resin Addition/Removal System with SuperLig ${ }^{\circledR}$ 659," WSRC-TR-2000-00303, Westinghouse Savannah River Company, Aiken, SC (2000).

Pitman EB, "Forces on Bins: The Effect of Random Friction," Phys. Rev. E 57, 3170-3175 (1997).

Poloski AP, PR Bredt, RC Daniel, and AE Sáez, “The contribution of frictional contacts to the shear strength of coarse glass bead powders and slurries", Rheologica Acta, published on the web, DOI: 10.1007/s00397-006-0105-3 (2006).

Press WH, BP Flannery, SA Teukolsky, and WT Vetterling, Numerical Recipes in FORTRAN 77: The Art of Scientific Computing, Cambridge University Press (1992).

Rabinowicz E, Friction and Wear of Materials, John Wiley and Sons, Inc. (1995).

Rapko BM and GJ Lumetta, "Status report on phase identification in Hanford tank sludges", PNNL-13394, Pacific Northwest National Laboratory, Richland WA (2005).

Sáez AE, AP Poloski, and RC Daniel, "A Continuum Constitutive Model for Cohesionless Granular Flows", Submitted to Chem. Eng. Sci. (2006).

Savage SB and K Hutter, "The motion of a finite mass of granular material down a rough incline", Journal of Fluid Mechanics 199, 177-215 (1989).

Sherwood JD, "Packing of spheroids in three-dimensional space by random sequential addition", J. Phys. A: Math. Gen. 30, L839-L843, (1997).

Shinohara K, "Fundamental and Rheological Properties of Powders," Chapter 4 in: Fayed ME, and Otten L (Eds), Handbook of Powder Science and Technology, Chapman and Hall (1997).

Slatter PT, “The Rheological Characterization of Sludges” Wat. Sci. Tech. 36, 9-18 (1997).

Steffe JF, Rheological Methods in Food Process Engineering, Freeman Press, East Lansing (1992).

Stewart CW, SA Hartley, PA Meyer, and BE Wells, "Predicting Peak Hydrogen Concentrations from Spontaneous Gas Releases in Hanford Waste Tanks," PNNL-15238, Pacific Northwest National Laboratory, Richland WA (2005).

Sukop MC and DT Thorne, Lattice Boltzmann Modeling: An Introduction for Geoscientists and Engineers, Springer (2005).

Tardos GI, S McNamara, and I Talu, "Slow and Intermediate Flow of a Frictional Bulk Powder in the Couette Geometry”, Powder Technology 131, 23-39 (2003). 
Terzaghi K, Theoretical Soil Mechanics, John Wiley and Sons, Inc., New York (1943).

Tingey J, J Gao, C Delegard, L Bagaasen, B Wells, "Physical Property and Rheological Testing of Actual Transuranic Waste from Hanford Single-Shell Tanks", PNNL-14365, Pacific Northwest National Laboratory, Richland WA (2005).

Tingey JM, "Vacuum drying of actual transuranic waste from Hanford tanks", PNNL-14670, Pacific Northwest National Laboratory, Richland WA (2004).

van Dillen T, Ion irradiation-induced anisotropic plastic deformation, $\mathrm{PhD}$ Thesis, FOM Institute for Atomic and Molecular Physics, Amsterdam, The Netherlands (2004).

Velikov KP, T van Dillen, A Polman, and A van Blaadren, "Photonic crystals of shapeanisotropic colloidal particles", Appl. Phys. Lett. 81, 838-840, (2002).

Zachariah MR and MJ Carrier, "Molecular dynamics computation of gas-phase nanoparticle sintering: A comparison with phenomenological models”, J Aerosol Sci 30, 1139-1151 (1999). 


\section{Appendix A}

\section{Vane rheograms for Dense Slurries of Glass Beads in Kaolin}

Suspensions 



\section{Appendix A - Vane rheograms for Dense Slurries of Glass Beads in Kaolin Suspensions}

The rheology of the suspending phase significantly influences granular medium rheology. Both models presented in Chapters 3 and 5 have granular rheology that depends on a buoyant density. In the most basic sense, the suspending phase moderates the intergranular normal stresses through bouncy, reducing the stress required for both mobilization and flows.

For dynamic flows, the numerical simulations by da Cruz et al. (2005) for the basis for the model presented in Chapter 5. These simulations only consider the dynamics of cohesion rigid spheres undergoing simple shear. While Jop et al. (2006) and Sáez et al. (2006) have extended da Cruz's simulations to three dimensional flows, neither of these approaches consider how the rheology of the suspending phase might influence the overall rheology of a dense granular slurry. This was observed to some extent in the study of the 203- $\mu$ m slurry in water (see Section 5.3.4), where a slightly higher $b$ constant was required to correct slurry dynamics as compared to dry glass bead dynamics. It is speculated that the increased $b$ derives from viscous dissipation of bead momentum by the suspending phase motion.

For the examination of granular rheology presented in Chapter 5, both systems tested had a Newtonian suspending phase: air in the case of the glass bead powders and water in the case of the glass bead slurry. In this appendix, vane rheographs for dense slurries of $203-\mu \mathrm{m}$ glass beads in a 35-wt\% Kaolin suspension are presented. The goal of these studies was to explore the influence of a non-Newtonian suspending phase, in particular, a Kaolin clay suspension, on slurry rheology.

As before, Potters Industries Spheriglass ${ }^{\circledR} 1922$ Type-A glass beads were used as the coarse granular fraction. The material properties of these glass beads are listed in Section 3.2. Slurries of glass beads in Kaolin suspensions were prepared by slowing loading the glass beads into a kaolinite matrix. The matrix was prepared by mixing deionized water with EPK Kaolin produced by Feldspar corporation to a loading of $35 w t \%$ and allowing clay-water mixture to hydrate for a period of one week. Three kaolin slurry were prepared in proportions of 1) 74-wt $\%$ glass beads - 26-wt\% kaolin matrix, 2) 64-wt\% glass beads - 36-wt\% kaolin matrix, and 3) 54-wt\% glass beads $-46-\mathrm{wt} \%$ kaolin matrix. These slurries were fully mixed prior to testing. Vane rheograms were measured using the same methodology outlined in Section 5.2.1. Once the shear strength measurement was completed, the vane was removed and the test sediment resuspended using a large spoon vertical bottom-to-top lifting motion (i.e., by ladling the slurry).

Figures A.1, A.2, and A.3 present the measured vane rheographs for the 54-wt $\%, 64-w t \%$, and 74-wt\% systems, respectively. Two major differences between the Kaolin slurry vane rheograms and rheograms for the dry glass bead powder and dry glass bead slurry in water: 1) vane torque appear to show similar dynamics behavior at all depths and 2) rheographs show a strong decrease from 0.01 to $0.1 \mathrm{rad} / \mathrm{s}$ for the densest slurry.

The local value of torque appears to be depth dependent, with deeper immersion depths generally showing higher vane torques. This indicates some lithostatic loading effects. However, the increases (or decreases) in torque with increased rotational rate are similar for all tested immersion depths. This contrasts with previous experiments, where deeper immersion depths show much larger increases in dynamic torque over shallow vane immersion depths, which show almost no increase in dynamic torque. Similar vane rheograph structure for all tested depths suggests that the suspending phase rheology (which is independent of depth) dominates the slurry 
rheology. On the other hand, the presence of the coarse fraction does add to the rheology. This is evidenced by the difference in torque ranges observed for different glass bead loadings. The $54-w t \%$ glass bead slurry shows torques ranging from $200-700 \mu \mathrm{N} \cdot \mathrm{m}$ whereas the $74-\mathrm{wt} \%$ slurry shows torques ranging from $500-2000 \mu \mathrm{N} \cdot \mathrm{m}$. It is unclear if the torque increase associated with higher concentrations of glass beads results from increased interparticle friction / collision or simply from increased viscous dissipation. The lack of significant change in the lithostatic slope at increasing rotational rates for the 74-wt\% slurry would appear to indicate the latter mechanism.

The large dip observed in the 74-wt\% slurry sets it apart from previously measured granular rheographs. This decrease appears to be a function of the glass bead concentration. This is evidenced by the fact that the 54-wt\% shows no dip and the 64-wt\% shows only a minor dip. It is speculated that the decrease is associated with shear thinning of the Kaolin matrix exaggerated by the presence of glass beads.

These studies indicate the importance of suspending phase rheology on the overall rheology of a granular suspension. They highlight a deficiency in the models presented in Chapters 3 to 5: the role of the suspending phase is not adequately considered. While the lack of dynamic granular interaction could be modeled using $b=0$, there is no established means of including the shear-thinning behavior of the Kaolin matrix and its interaction with the granular medium into the model of Sáez et al. (2006). One way of accomplishing this would be to treat the apparent granular viscosity [Eq. 4.11] as additive to the suspending phase rheology, with the frictional / collosional contributions properly moderated by slurry concentration (i.e., properly lowered at lower concentrations). Despite this deficiency in the current continuum model, it still represents a robust step toward treating flow dynamics in granular media.

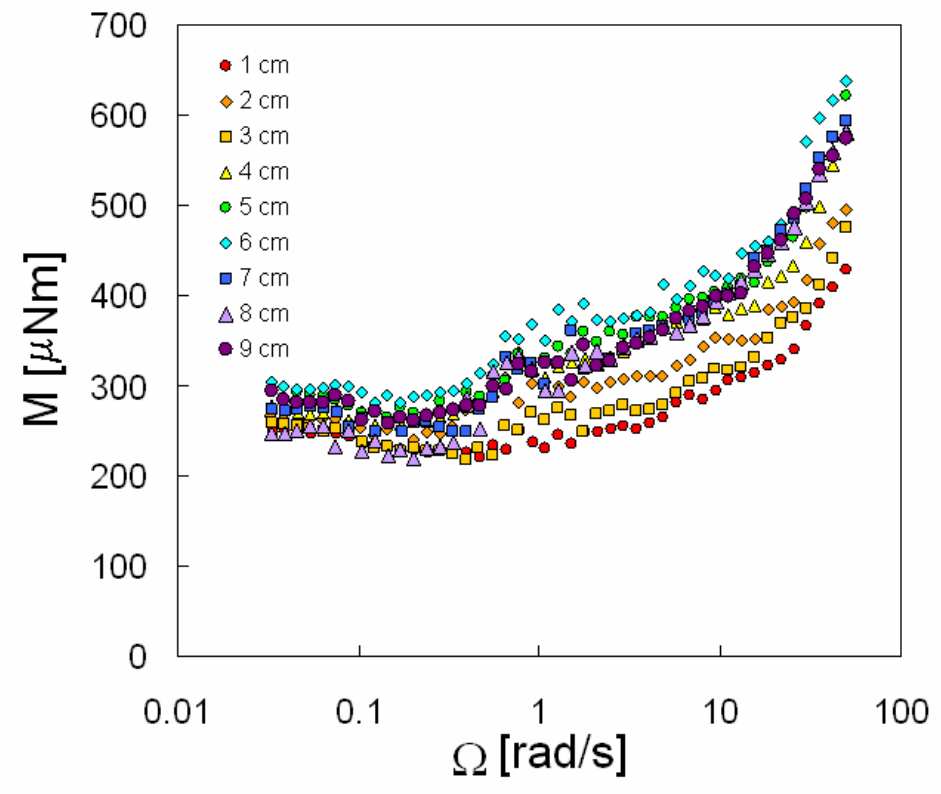

Figure A.1. Vane rheographs for a 54-wt\% glass bead - 46-wt $\%$ kaolin matrix slurry over vane immersion depths of $1 \mathrm{~cm}$ to $9 \mathrm{~cm}$ 


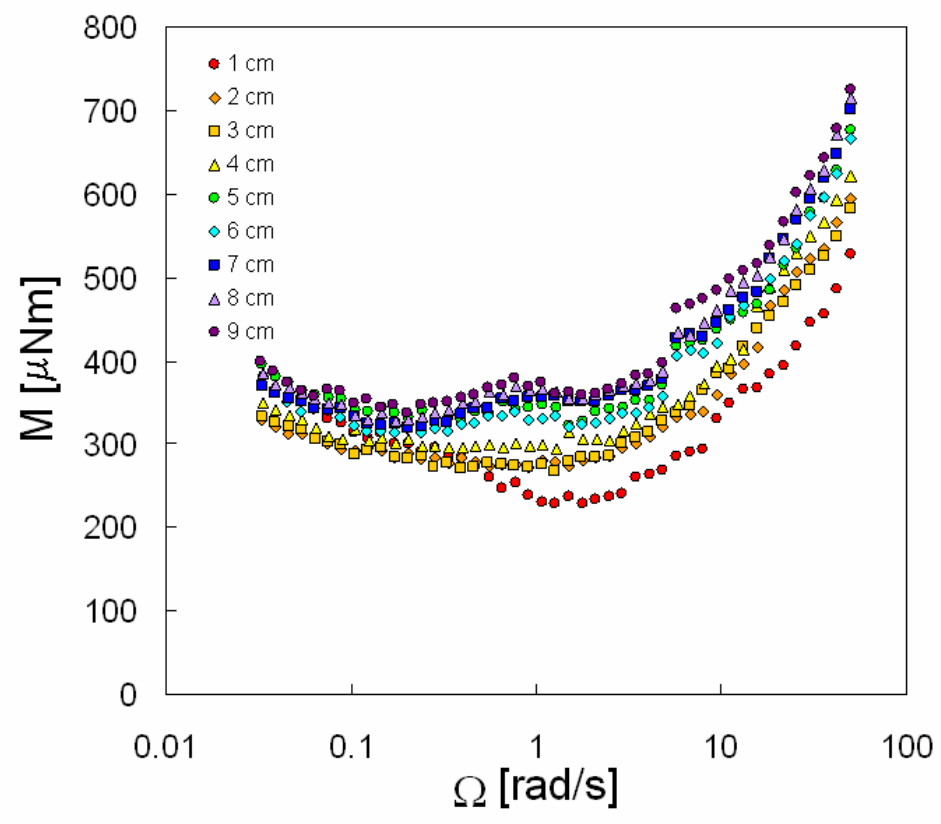

Figure A.2. Vane rheographs for a 64-wt $\%$ glass bead - 36-wt $\%$ kaolin matrix slurry over vane immersion depths of $1 \mathrm{~cm}$ to $9 \mathrm{~cm}$

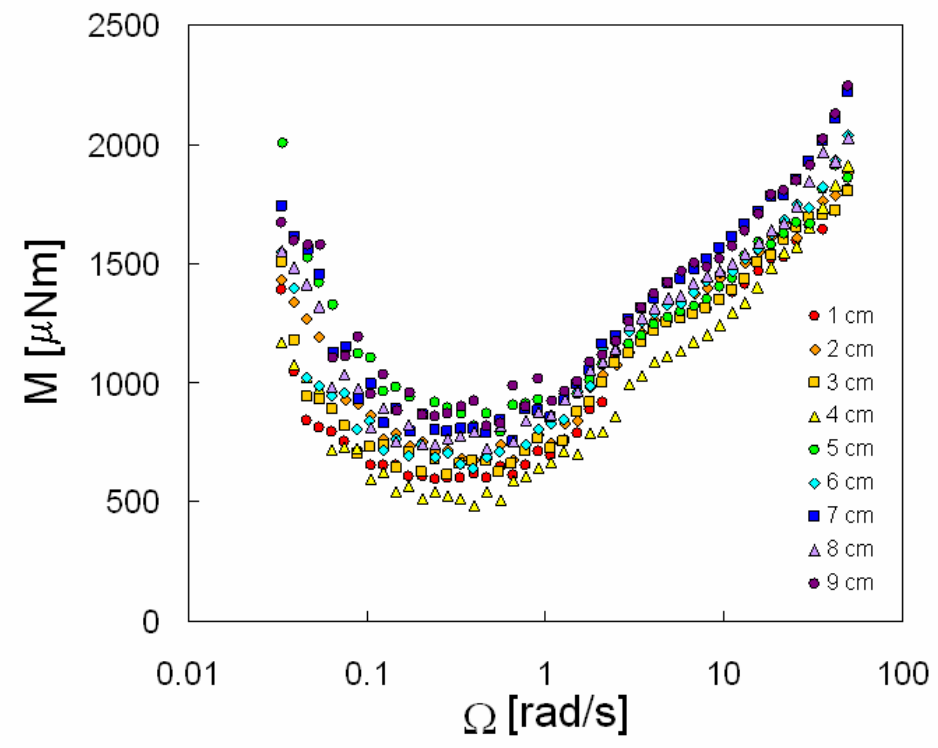

Figure A.3. Vane rheographs for a 74-wt $\%$ glass bead - 26-wt $\%$ kaolin matrix slurry over vane immersion depths of $1 \mathrm{~cm}$ to $9 \mathrm{~cm}$ 



\section{Appendix B}

\section{University of Washington Collaborative Effort Update}





\section{Appendix B - University of Washington Collaborative Effort Update}

Note: This appendix contains a year-three research progress summary submitted by Dr. John Berg at the University of Washington to the DOE

Work relating to the development of relationships between colloidal parameters and bulk physical properties was accomplished through a collaborative effort between PNNL and Professor John C. Berg from the University of Washington [DOE grant DE-FG02-04ER63796]. Year three of this continuing collaboration has yielded significant progress in the development of fundamental understanding of the behavior of Hanford transuranic, TRU, waste sludge. In the most recent study, data have been collected that will determine the applicability of a new simulation protocol to the waste sludge removal project. A second study investigated the link between a new micromechanical measurement technique and the bulk rheological properties of gelled slurries. Also, it has come to light throughout the course of this work that an understanding of the behavior of colloids intercalated within a gel network could prove indispensable in the removal, packaging, shipping, and storing of TRU wastes. The work done over the past project year has provided considerable advancement toward the goal of developing fundamental understanding of the TRU sludge suspensions by correlating their macroscopic properties with particle interactions at a colloidal scale in representative media. Year three accomplishments and milestones are summarized as follows and elaborated below:

- Papers appeared:

1. A.Y. Huang, J.C. Berg, High-salt stabilization of Laponite clay particles, J. Colloid Interface Sci. 296 (2006) 159-164.

2. P.B. Laxton, J.C. Berg, Relating clay yield stress to colloidal parameters, J. Colloid Interface Sci. 296 (2006) 749-755.

- Presentation given: "Relating clay yield stress to colloidal parameters", P.B. Laxton, 80th ACS Colloid and Surface Science Symposium, Boulder, CO, June 20, 2006.

- Manuscript submitted: P. B. Laxton, J. C Berg "Investigation of the link between micromechanical interparticle bond rigidity measurements and macroscopic shear moduli of colloidal gels", Colloids and Surfaces A, submitted September 1, 2006.

- Manuscript in preparation: D. Rector, P.B. Laxton, J.C. Berg, on the comparison of Lattice-Boltzmann simulations to sheared particle aggregate size distribution data for submission to Colloids and Surfaces A.

- Arrangements have been made for a collaboration with Professor Wilhelm Oppermann of the Clausthal University of Technology.

\section{B.1 Summary of Current Results}

A method of simulating the aggregation behavior of colloids in a shear field is being investigated at PNNL [D. Rector, PNNL, Hanford, WA. Private communication, 2006]. The simulation uses the Lattice-Boltzmann method to obtain system hydrodynamics. This method is an improvement on conventional computational fluid dynamics in that it allows for parallel processing, reducing run time, as well as the inclusion of additional forces, e.g. surface forces. Simulations of 
this type have been used extensively for calculating transport in porous media. At PNNL the Lattice-Boltzmann method has been extended to colloidal suspensions. The direct quadrature method of moments [D.L. Marchisio, R.O. Fox, J. Aerosol Sci. 36 (2005) 43] is used to reduce computational requirements in aggregate population balance calculations. It is hoped that the simulation accurately predicts aggregate size distributions and ultimately shear stress-strain rate relationships for steady-state suspensions in a shear field given the system's colloid properties and shear rate. The accuracy of the predictions made by this simulation had not been tested against real systems. The objective was therefore to provide an experimental data set that could be used to compare with simulations.

The data set provided to PNNL consisted of aggregate particle size distributions as a function of colloidal interaction parameters and applied shear rate. Systems of aqueous titania suspensions were aggregated at a controlled shear rate by adding known amounts of salt. These systems were convenient since, under the conditions used, particle interaction potentials can be calculated according to DLVO theory. Particle interactions as calculated using DLVO theory require the knowledge of certain colloidal parameters including the zeta potential. This quantity was measured using a Brookhaven Zeta-PALS.

Various controlled shear rates were obtained using a Physica MCR-300 rheometer. Stable titania suspensions at $\mathrm{pH} 10$ were sheared at controlled rates with the rheometer. Then salt solution was injected into the sample by syringe so that the aggregation process occurred within the shear field. Various amounts of salt were used in order to obtain particle interaction potentials ranging from repulsive to strongly attractive. The aggregation process was allowed to reach steady-state. It was assumed that a steady-state was achieved when the recorded shear stress at the given shear rate became constant with time.

Samples were then taken for particle size determination. These samples were immediately diluted in order to prevent further aggregation. Particle size distributions were determined by a settling technique which uses light obscuration to track sedimentation rate then calculates size based on Stokes Law. The equipment used for this measurement was a Horiba CAPA-500.

A matrix of particle size distributions of systems with various zeta potentials aggregated at different shear rates with two different solids contents was collected using these measurement techniques. The full data set was then provided to PNNL for comparison with simulation results. A joint publication for submission to the journal Colloids and Surfaces A is in preparation.

Another method relating the bulk properties of colloidal systems to microscopic colloidal parameters has been independently explored with experimental data collection. The ability to measure a previously inaccessible quantity, the interparticle bond strength, has allowed for a simple calculation of colloid gel modulus. It has been recognized since the 1980s that the shear modulus of colloid gels follows a scaling law with volume fraction [R. Buscall et al. J Chem Soc, Faraday Trans 1. 84 (1988) 4249]. Pantina and Furst have recently developed a technique to directly measure the appropriate pre-factor, known as the bond rigidity, for the scaling law which allows for the calculation of gel modulus [J.P. Pantina, E.M. Furst, Langmuir, 22 (2006) 5282]. Although Pantina and Furst provided bond rigidity measurements and therefore predictions of bulk modulus, they did not provide experimental bulk moduli as confirmation of the technique. The goal of this work was therefore to measure bulk colloid gel moduli for comparison with the predicted values.

Using micromechanics Pantina and Furst measured the bond rigidity of a series of linear colloidal aggregates. They used optical trapping with laser tweezers to create controlled linear aggregates 
of monodisperse latex spheres suspended in aqueous systems with various ion type and concentration. They then used a three point bending test, employing the laser tweezers, to measure the bond rigidity of these aggregates. The bond rigidity divided by the particle radius is the prefactor which makes the gel modulus scaling law an equation. Using the measured bond rigidity, gel modulus was then calculated for bulk systems analogous to the aggregates investigated by Pantina and Furst.

In order to calculate gel modulus some system parameters must be known. The particle volume fraction, aggregate fractal dimension, gel backbone fractal dimension, the particle radius and bond rigidity must all be known. Volume fractions of the systems studied were determined by thermal gravimetric analysis. Aggregate fractal dimension was measured with static light scattering. The gel backbone fractal dimension was taken to be 1.1 in accord with Meakin [P. Meakin, J. Phys. A, 17 (1984) L975]. An average hydrodynamic particle radius was determined using photon correlation spectroscopy. Finally, as mentioned previously, the bond rigidity was reported by Pantina and Furst. The gel shear modulus was calculated from these parameters. The bulk modulus of identical systems was then measured for comparison.

Shear moduli of poly (methyl methacrylate), PMMA, spheres in aqueous media were measured using a Physica MCR-300 rheometer. The 1.5- $\mu$ m diameter PMMA spheres used in this study match those used by Pantina and Furst as closely as possible; they were provided by the same source, Bangs Laboratories, Inc. Suspensions of these particles were gelled using salt types and concentrations matching those used in the Pantina and Furst study. In this way it was ensured that the reported bond rigidities could be used in calculating moduli of the systems studied. After gelation, moduli were measured in oscillatory mode using a rheometer tool with significant surface roughness. A high frequency amplitude sweep was performed and the storage modulus at the lowest measurable amplitude was taken as the shear modulus. The surface roughness of the measuring tool is crucial for rheological characterization of colloidal systems since they experience slip which results in erroneous data when using smooth tools.

Calculated and measured values of shear modulus for these PMMA gels agree to within a factor of two. The general trends predicted by the calculated moduli were observed for the bulk systems. These were seen to be a significant contribution to the field and have been submitted for publication in the journal Colloids and Surfaces A.

Over the course of the third year of this project substantial progress has been made. Fundamental studies of systems that simulate transuranic waste behavior have been performed. Future work pertinent to this project has been identified, as described below. The studies performed have resulted in significant contributions to the field of colloid science as well as produced results that may prove important in handling actual waste materials. The work over the course of this past year has been significant and proved to be sufficient to be the subject of two journal articles.

\section{B.2 Future Work}

The knowledge gained over the course of this project on the subject of relating macroscopic flow properties to colloidal scale particle interactions can be used in developing processes and designing equipment. A concern that is not addressed by the work completed is the formation of dense sediment cakes within process equipment upon cessation of flow which could lead to clogs. Given the high level of radioactivity involved in the waste materials to be handled, a serious health and safety hazard could be generated simply by turning off a pump for too long. Avoiding this scenario would be crucial to ensuring the safety of the operators of the waste handling equipment. One way to prevent this would be to add a polymer gel to the waste sludge. 
The flow properties of polymer gels are highly tunable. A gel could be made in such a way as to flow easily yet set quickly and strongly enough to prevent particle sedimentation and thus caking and clogging. Knowledge of colloid behavior within polymer gels would be useful in formulating such a system. A literature review of this subject reveals a gap in the knowledge base of the field. This gap appears to exist due, in part, to the fact that measurement techniques appropriate for studies in this area have been developed only recently. Although there was not time to perform studies in this area during year 3 of this project, many questions remain to be answered. A three month visit Professor Wilhelm Oppermann's research laboratory at Clausthal University of Technology in Clausthal, Germany has been planned for the upcoming fall in order to gain expertise in novel polymer gel characterization techniques. These techniques will be used in the following year, approximately 12/06 through 11/07, in order to perform investigations into the fundamental behavior of colloids intercalated into polymer networks. This work will be accommodated by way of a no cost extension to this project which has been institutionally approved. 